\title{
Role of binary metal chalcogenides in extending the limits of energy storage systems: Challenges and possible solutions
}

\author{
Muhammad Yousaf ${ }^{1,2}$, Ufra Naseer ${ }^{3}$, Imran Ali $^{2}$, Yiju Li $^{1}$, Waseem Aftab ${ }^{1}$, Asif Mahmood ${ }^{4}$, \\ Nasir Mahmood ${ }^{5}$, Peng Gao ${ }^{1^{*}}$, Yinzhu Jiang ${ }^{2,6^{*}}$ and Shaojun Guo ${ }^{1^{*}}$
}

\begin{abstract}
Binary metal chalcogenides (BMCs) have shown better electrochemical performance compared with their mono metal counterparts owing to their abundant phase interfaces, higher active sites, faster electrochemical kinetics and higher electronic conductivity. Nevertheless, their performance still undergoes adverse decline during electrochemical processes mainly due to poor intrinsic ionic conductivities, large volume expansions, and structural agglomeration and fracture. To tackle these problems, various strategies have been applied to engineer the BMC nanostructures to obtain optimized electrode materials. However, the lack of understanding of the electrochemical response of BMCs still hinders their large-scale application. This review not only highlights the recent progress and development in the preparation of BMC-based electrode materials but also explains the kinetics to further understand the relation between structure and performance. It will also explain the engineering of BMCs through nanostructuring and formation of their hybrid structures with various carbonaceous materials and three-dimensional (3D) templates. The review will discuss the detailed working mechanism of BMC-based nanostructures in various electrochemical energy storage (EES) systems including supercapacitors, metal-ion batteries, metal-air batteries, and alkaline batteries. In the end, major challenges and prospective solutions for the development of BMCs in EES devices are also outlined. We believe that the current review will provide a guideline for tailoring BMCs for better electrochemical devices.
\end{abstract}

Keywords: binary metal chalcogenides, nanomaterials, energy storage devices, supercapacitors, batteries

\section{INTRODUCTION}

The non-renewable fossil-based energy sources, including coal, oil and gas, are decreasing rapidly due to rising energy demand, and have adverse effects on climate and environment, which have provoked the development of renewable energy sources (wind, solar, geothermal, etc.). However, the full utilization of these energy resources cannot be exploited, without developing low cost, highly efficient and environmentally friendly electrochemical energy storage (ESS) systems to regulate the intermittent output. Among various electrochemical energy storage (EES) systems, supercapacitors (SCs), metal-ion batteries (MIBs) including lithium-ion batteries (LIBs), sodium-ion batteries (SIBs), potassium-ion batteries (PIBs) and multi-ion batteries (multi-IBs), metal-air batteries (MABs), and rechargeable alkaline batteries (RABs) are very promising [1-9]. Among these, LIBs are already commercialized but facing many challenges such as high cost, geopolitical issue and non-uniform distribution of lithium, and relatively low energy and power density of commercialized graphite anode in LIBs. Poor rate performance and low cycling stability owing to the slow reaction kinetics of larger ions ( $\mathrm{Na}, \mathrm{K}$, and multi ions) in SIBs, PIBs, and multi-IBs are also limitations in their commercialization [10-17]. In the case of SCs, inferior energy density and low rate capability are critical issues [18-20]. The sluggish oxygen reduction reaction (ORR) and oxygen evolution reaction (OER), bifunctional electrocatalysts with unsatisfactory activities for both ORR and OER, large overpotential, and insulating nature of discharge product in MABs are also very challenging. Therefore, to put these EES systems into practical applications, electroactive materials with low cost and high electrochemical activity are required. In this context, a lot of attention has been paid to designing innovative and cost-effective electrochemical active materials with emphasis on improving the performance of these EES systems.

Currently, transition metal chalcogenide (TMC)-based materials play a vital role in the field of EES owing to their large abundance, intrinsic metallic property, and better mechanical and thermal stability [21-23]. TMCs usually possess higher electrical conductivity than metal oxide counterparts. Also, the bond energy of M-S/Se bond in TMCs is lower than that of the $\mathrm{M}-\mathrm{O}$ bond in metal oxides, leading to rapid reaction kinetics during the electrochemical conversion reaction [24,25]. Particularly, binary metal chalcogenides (BMCs) that are composed

\footnotetext{
${ }^{1}$ School of Materials Science and Engineering, International Center for Quantum Materials and Electron Microscopy Laboratory, School of Physics, Peking University, Beijing 100871, China

${ }^{2}$ ZJU-Hangzhou Global Scientific and Technological Innovation Centre, Zhejiang University, Hangzhou 311200, China

${ }^{3}$ State Key Laboratory of Resources and Environment Information System, Institute of Geographical Sciences \& Natural Resources Research, Chinese Academy of Sciences, Beijing 100871, China

${ }^{4}$ School of Chemical and Biomolecular Engineering, The University of Sydney, Darlington, Sydney, New South Wales, 2006, Australia

${ }^{5}$ School of Engineering, RMIT University, 124 La Trobe Street, Melbourne, Victoria, 3001, Australia

${ }^{6}$ School of Materials Science and Engineering, State Key Laboratory of Clean Energy Utilization, Zhejiang University, Hangzhou 310027, China

*Corresponding authors (emails: p-gao@pku.edu.cn (Gao P); yzjiang@zju.edu.cn (Jiang Y); guosj@pku.edu.cn (Guo S))
} 
of two different transition metals have attracted significant interest in the past few years due to their abundant redox-active sites and rich multi-electron reaction. In addition, these BMCs usually offer better electrical conductivity than mono TMCs due to lower activation energy for the transfer of electrons between the cations [26]. More significantly, BMCs also have better thermal and mechanical stabilities than their corresponding metal oxides [27]. Therefore, BMCs have been extensively investigated as electrode materials for SCs, MIBs, MABs, and RABs. However, the electrochemical performance of BMCs electrodes is still rigorously limited by structural fracture and agglomeration during the electrochemical reactions. For instance, the shuttle effect of lithium/sodium polysulfides/selenides for binary metal sulfides/selenides as anodes for LIBs/SIBs remains a big challenge for practical application. Most BMCs suffer from extensive volume change upon sequential alloying and conversion reactions that leads to large mechanical strain and results in pulverization of electrode materials and rapid capacity/capacitance fading. Moreover, they possess unsatisfactory intrinsic electronic conductivity, inadequate number of accessible active sites, blocked channels for ion transportation, and the consequent low rate capabilities in energy storage devices. In addition, big voltage hysteresis (voltage gap) between charge/discharge voltage profiles due to irreversible phase reaction usually results in low-energy efficiency which is unacceptable in practical applications [28].

To address the above-mentioned obstacles, extensive efforts have been devoted to the synthesis of various nanostructures of BMCs with emphasis on the optimization of electrode materials for improving the electrochemical performance. The development of porous hollow structures is an intriguing strategy, because they assist in electron/ion transport, enhance the electrochemical activities and improve the mechanical and structural stability during electrochemical processes. Engineering the nanocomposites of two or more materials has been another intriguing strategy that can combine the advantages of individual counterparts, overcome the associated weakness/ shortcomings and optimize their electrochemical performance. For instance, nanocomposites of BMCs with carbonaceous materials by hybridization not only enhance the conductivity and structural integrity but also produce novel functions for the enhanced applications. Moreover, heteroatoms doping in BMCs can modulate their electrical and structural properties due to the distortion, and expansion/contraction in lattice, which offer facile pathways for ion penetration into the host materials and thus boost battery performance. The above-mentioned approaches have been extensively studied to fabricate the BMC-based electrodes and efficiently enhance the electrochemical performance. However, systematic studies of these strategies for BMCs in EES have not been discussed and reviewed. Therefore, it is an urgent need to timely highlight the recent developments on BMC-based electrode materials for EES systems.

This review article targets the recent progress on the fabrication of nanostructural design of BMCs and their hybrids that have been used to improve the electrochemical performance for EES systems (Fig. 1). In the first part, we highlight the challenges of BMC electrodes in EES systems. Then we present current advances in nanostructural engineering of BMCs including zerodimensional (0D), 1D, 2D, and 3D hierarchical porous and hollow nanostructures. Moreover, we also introduce the doping strategy, and various nanohybrids of BMCs with different car-

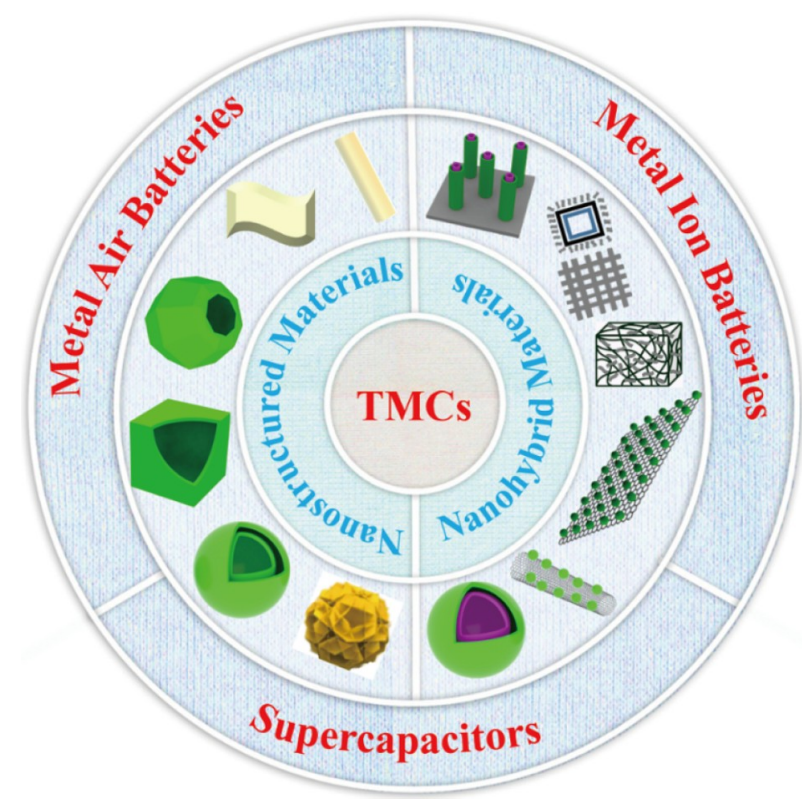

Figure 1 Schematic illustration of various nanostructured and hybrid designs of BMCs for EES devices.

bon scaffolds, and 3D conductive substrates. Next, we thoroughly investigate the applications of BMC architectures in SCs, MIBs, MABs, and RABs. Finally, we provide a brief summary and the challenges associated with BMC materials and their prospective solutions. Note that for simplicity, the BMCs presented in this review deals with single-phase BMCs and exclude other chemistries based on mixed phase chalcogenides.

\section{ADVANTAGES AND CHALLENGES OF BMCs ELECTRODES}

BMCs generally possess significant structural and chemical advantages over monometallic chalcogenides, which can improve electrochemical performances. It has been suggested that BMCs generally have higher electronic conductivity owing to relatively smaller bandgap and lower activation energy for the transfer of electrons between cations, leading to higher electrochemical properties [29]. For example, the electronic conductivity of $\mathrm{NiCo}_{2} \mathrm{~S}_{4}$ is at least two times higher than that of the corresponding single nickel sulfides and cobalt sulfides [29]. Moreover, $\mathrm{NiCo}_{2} \mathrm{~S}_{4}$ has a spinel structure, where $\mathrm{Co}$ and $\mathrm{Ni}$, respectively, occupy octahedral and tetrahedral positions that can combine the benefits of multicomponent and multi-phases [30]. The presence of these multi-phases in BMCs generates ample phase boundaries and small crystalline domains, assisting to circumvent solid-state diffusion and facilitating rapid kinetics for ionic diffusion [31]. On the other hand, the presence of multiple valences of cations in BMCs leads to more reduction and oxidation reaction sites and multi-electron reactions than mono-chalcogenides, and hence higher electrochemical activity. Moreover, the chemical composition, structure and valence of BMCs can be precisely controlled, and their composites with oxides, hydroxides, sulfides, selenides and phosphides are known to be stable due to multiple valences.

Recently BMCs have made promising progress in EES systems due to their extraordinary properties; however, it is still an emerging research field and has many challenges that need to be 
addressed. These challenges are originated from low conductivity, structural/phase change, huge volume variation during conversion/alloying process, undesirable reactions, large voltage hysteresis, and polarization. Some of the challenges associated with BMC-based electrodes for EES systems are presented as follow.

\section{Low intrinsic conductivity}

The electrical conductivity of electrode materials is an important factor to achieve high electrochemical performance. Most of the BMC-based electrode materials possess low intrinsic electrical conductivity, which reduces the diffusivity of ions and results in degradation of capacity and poor rate performance. In addition, due to poor electrical conductivity, the active materials cannot use all of their active sites, as no routes exist for the transfer of electrons between the active site and external electrical circuit. This issue can be resolved by incorporation of carbonaceous materials (e.g., graphene, carbon nanotubes (CNTs), and amorphous carbon) or by doping of heteroatoms.

\section{Unwanted surface reactions}

During the electrochemical process, the final discharge products may react adversely with an electrolyte that badly affects the lithium, sodium, or potassium storage. For example, the conversion reaction between alkali metal (lithium, sodium, etc.) and BMCs in MIBs leads to the formation of sodium sulfides/polysulfides/selenides, which may react adversely with electrolytes, especially carbon-ester-based or ester-based electrolytes, resulting in capacity fading [32]. Moreover, the large surface area of nanostructured BMCs may create unwanted surface reactions. These issues can be mitigated by surface coating.

\section{Morphological changes and structural/phase transitions}

The electrochemical performance of BMC-based electrodes in EES systems strongly depends on different morphologies. Various morphologies of BMCs behave differently to $\mathrm{Li}, \mathrm{Na}$, or $\mathrm{K}$ storage. The change in morphology of the electrode materials during the electrochemical process causes the agglomeration of newly discriminated product, leading to electrical contact damage, and results in the annihilation of battery operation and safety issues. Moreover, the morphological change also causes the new contact of the electrode surface with an electrolyte, which results in the formation of fresh solid electrolyte interphase (SEI) films, leading to severe irreversible capacity loss. Similarly, most BMCs show phase changes during the electrochemical process, particularly in the first two cycles. The structure or phase of some BMCs could not be regenerated after the initial discharging process. The phase transition, irreversible structural changes, and lattice distortion can cause poor electronic conductivity and have a ruthless effect on the Coulombic efficiency and cyclic performance.

\section{Volume expansion}

The large volume changes during conversion or alloying charge storage mechanisms in BMC electrode materials are due to the participating of the huge amount of $\mathrm{Li}^{+} / \mathrm{Na}^{+} / \mathrm{K}^{+}$ions in the electrochemical reaction, which may cause the serious volume increase and consequently pulverization to take place. Thus, the mechanical strains are produced in the electrodes which break the SEI film, leading to drastic capacity fading and degradation of cycling life [33]. Furthermore, due to consecutive pulveriza- tion during the continous cycling process, dead areas are created that are electrically isolated from the conductive agents and result in capacity fading. In addition, the morphological changes or volume expansion bring the fresh surface of active material in contact with electrolyte, leading to the formation of new SEI film which results in an irreversible capacity loss. Use of conductive and soft matrix can be an effective strategy to address these issues.

\section{Voltage hysteresis}

Mostly, the conversion reaction is involved in BMCs during the charge storage mechanism that may exhibit the large voltage hysteresis between discharge and charge voltage profiles and the resultant low energy efficiency. The voltage hysteresis can be alleviated by rational design and optimization of the BMC microstructure and electrode architecture.

\section{Polarization}

The monovalent alkali ions (e.g., $\mathrm{Li}^{+}, \mathrm{Na}^{+}, \mathrm{K}^{+}$) can be easily reversibly intercalated/de-intercalated into BMCs during the charging/discharging process. However the multivalent alkali ions like $\mathrm{Mg}^{2+}, \mathrm{Al}^{3+}$ can polarize the host materials due to the higher charge density of multivalent ions (e.g., $120 \mathrm{C} \mathrm{mm}^{-3}$ for $\mathrm{Mg}^{2+}$ vs. $52 \mathrm{C} \mathrm{mm}^{-3}$ for $\mathrm{Li}^{+}$), the consequent reduction in ion mobility and poor rate performance.

\section{ENGINEERING STRATEGIES TO IMPROVE THE PERFORMANCE OF BMCs}

Nanostructure engineering has been proven an effective strategy to improve the electrochemical performance of BMCs for EES devices [34]. Electrode materials of BMCs obtained from nanostructure engineering provide many potential advantages such as high surface-to-mass/volume ratio, small grain size, good electronic conductivity and ionic transport, and unique nanoarchitecture. Specifically, the large surface area enables the access to all electrochemical active sites, assists the full penetration of electrolyte, and decreases the diffusion resistance of the electrolyte, leading to fast ion diffusion and high electrochemical performance. The nanometer-scale-sized particles facilitate the large contact area between the electrode/electrolyte, provide a short path length for electronic/ionic transport, and result in high rate capability. These features make nanostructured BMC-based electrodes an attractive solution for better electrochemical performance. Until now, nanostructured BMCs with various particle morphologies, such as $0 \mathrm{D}$ nanoparticles, 1D nanotubes/nanorods/nanobelts, 2D nanoplates/ nanosheets, and 3D hierarchical structures, have been broadly investigated as electrode materials for EES devices. These various structural features of nanostructured BMCs result in different electrochemical properties. For example, $0 \mathrm{D}, 1 \mathrm{D}$, and $2 \mathrm{D}$ nanostructures have the advantage of accelerating charge transport. However, these conventional nanostructures may agglomerate during the electrochemical process, resulting in poor cyclic stability. Further, high surface area results in excessive reaction with the electrolyte, hence leading to the formation of thick SEI which results in not only slow diffusion but also irreversible consumption of electrolyte, ultimately causing poor Coulombic efficiency. These issues can be addressed by fabricating 3D hierarchical porous/hollow nanoarchitectures with a stable structure. The 3D hierarchical porous/hollow structures consisting of $0 \mathrm{D}, 1 \mathrm{D}$, and $2 \mathrm{D}$ as building blocks combine the 
characteristics of nano-dimensional structures and micrometer architectures, which can prevent the agglomeration, improve the structural integrity, and accommodate the volume variation during the electrochemical process. To further improve the electrochemical performance of BMCs, nanostructured hybrids of BMCs with carbonaceous materials, polymers, nickel foam (Ni-F) and metal compounds, etc. have also been extensively explored. These nanohybrid hierarchical structures not only take the advantage of various nanostructures $(0 \mathrm{D}, 1 \mathrm{D}, 2 \mathrm{D}$, or $3 \mathrm{D})$, but also increase the electronic conductivity, enhance the naked electroactive sites, prevent the agglomeration of interparticle, and improve the stability of BMCs. In the following section, the synthetic methods for the fabrication of conventional and $3 \mathrm{D}$ porous/hollow nanostructured BMCs, BMC-based nanohybrids, and heteroatom-doped BMCs are introduced and discussed.

\section{Morphology control of nanostructured BMCs}

OD to $2 D$ nanostructures of BMCs

Morphology control by nanostructure engineering has been broadly pursued to improve the electrochemical reaction kinetics and buffer the volume variations of BMCs. In the past decade, many conventional nanostructured BMCs with diverse shapes, sizes, and morphologies including nanoparticles, nanorods, nanoneedles, nanosheets, have been widely fabricated and used as electroactive materials for EES devices. For instance, nanoparticles of various BMCs with different shapes including spheres [35-37], or cubohexa-octahedral [38] were synthesized. In the early stage, solid-state reactions at elevated temperatures $\left(>300^{\circ} \mathrm{C}\right)$ have been used to synthesize the BMC-based nanoparticles, in which a chalcogen and two transition metals blended together and subsequently were heated at an elevated temperature in vacuum or inert gas which lead to agglomerated particles, reduced surface area, and less control over desired morphology and size [39]. Later on, the BMC nanoparticles are prepared at a lower temperature using hydrothermal/ solvothermal [36], chemical solution precipitation [40] and in combination with calcination. The calcination improves the crystallinity and removes $\mathrm{O}_{2}$ from the structure, leading to improved porosity. For instance, non-agglomerated and highly dispersed $\mathrm{CuCo}_{2} \mathrm{~S}_{4}$ nanoparticles with quasi-spherical shape and an average diameter of $10 \pm 14 \mathrm{~nm}$ were synthesized by a solution-based route at $200^{\circ} \mathrm{C}$ (Fig. 2a, b) [40]. The $\mathrm{CuCO}_{2} \mathrm{~S}_{4}$ was large single crystalline, and the lattice spacing detected by highresolution transition electron microscopy (HRTEM) was $0.33 \mathrm{~nm}$ that corresponded to (220) plane of spinel-type
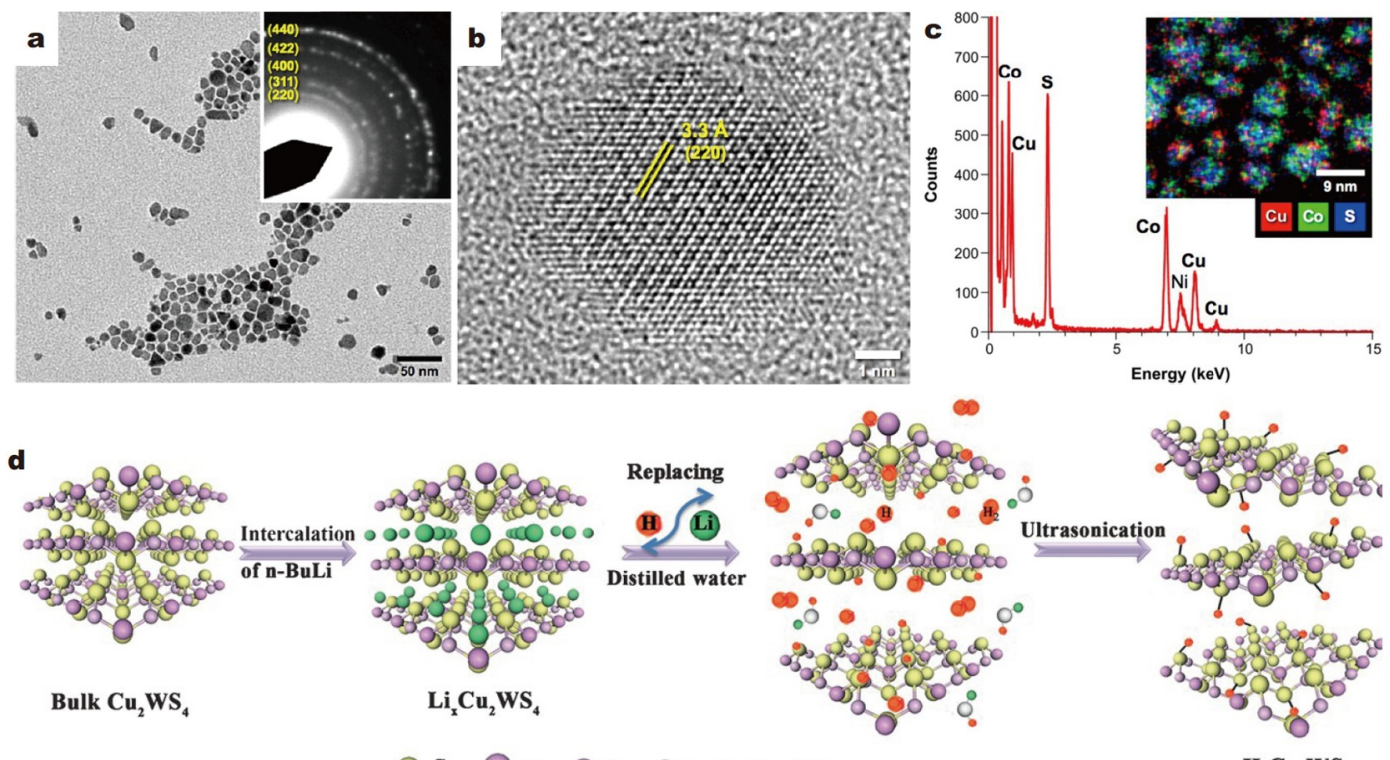

Os Ow OCu Oo OLi $\bullet$ H

$\mathrm{H}_{\mathrm{y}} \mathrm{Cu}_{2} \mathrm{WS}_{4}$
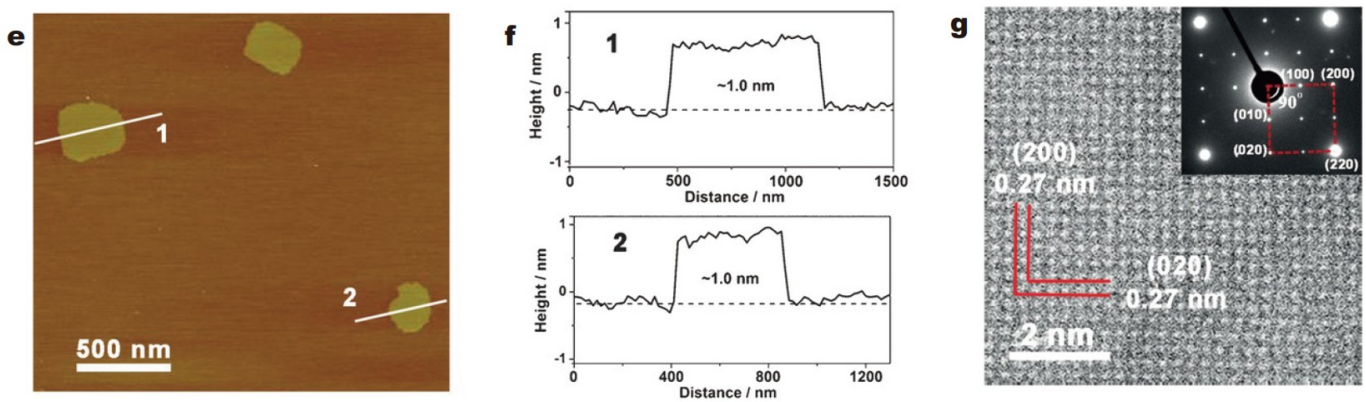

Figure 2 Formation of various solid nanostructured BMCS: (a-c) $\mathrm{CuCo}_{2} \mathrm{~S}_{4}$ nanoparticles. (a) TEM image with corresponding selected area electron diffraction (SAED) pattern (inset) for $\mathrm{CuCo}_{2} \mathrm{~S}_{4}$ nanoparticles. (b) HRTEM image of a representative $\mathrm{CuCo}_{2} \mathrm{~S}_{4}$ nanoparticle. Energy-dispersive X-Ray spectroscopy (EDS) spectrum and scanning TEM-EDS (STEM-EDS) elemental map (inset) of $\mathrm{CuCo}_{2} \mathrm{~S}_{4}$ nanoparticles. Reprinted with permission from Ref. [40]. Copyright 2016, American Chemical Society. (d-f) $\mathrm{Cu}_{2} \mathrm{WS}_{4}$ nanosheets. (d) Formation of hydrogenated $\mathrm{Cu}_{2} \mathrm{WS}_{4}$ nanosheets. (e) Atomic force microscopy (AFM) image of the as-obtained nanosheets. (f) Corresponding height diagram. (g) HRTEM image and SAED pattern of the as-obtained nanosheets. Reprinted with permission from Ref. [54]. Copyright 2016, John Wiley and Sons. 
$\mathrm{CuCo}_{2} \mathrm{~S}_{4}$ (Fig. 2c). However, 0D nanoparticles may agglomerate during the electrochemical process, resulting in poor device performance. Therefore, $1 \mathrm{D}$ and $2 \mathrm{D}$ nanostructures can be more effective as they can prevent agglomeration while keeping the same advantages as 0D nanostructures [41]. Moreover, 1D nanostructures show additional distinct properties of accelerated conduction pathways for both ions and electrons. Many 1D and 2D nanostructured BMCs, including nanobelts [42], nanorods [43-45], nanowires [46], and 2D nanosheets/nanoplates [47-50] have been synthesized using the most common methods such as hydrothermal/solvothermal and chemical solution approaches. As a representative example, a hierarchical iron-cobalt selenide $\left(\mathrm{Fe}_{x} \mathrm{Co}_{3-x} \mathrm{Se}_{4}, 0 \leq x \leq 3\right)$ with tunable nanostructure and morphology was synthesized by adjusting the stoichiometry ratio of Fe:Co using the most common hydrothermal approach followed by a selenization process [51]. The resulting $\mathrm{FeCo}_{2} \mathrm{Se}_{4}$ exhibited better electrical conductivity, more electroactive sites, higher intrinsic reactivity and ultra-high performance.

Moreover, the layered $\mathrm{BMC}$ nanosheets with semiconductor behavior with the formula $\mathrm{Cu}-\mathrm{M}-\mathrm{X}_{4}(\mathrm{M}=\mathrm{Mo}, \mathrm{W}, \mathrm{Sb}, \mathrm{X}=\mathrm{S}, \mathrm{Se})$ have also been prepared for electrochemical applications $[47,52,53]$. However, less attention has been given to their fascinating electronic structure. $\mathrm{Hu}$ et al. [54] presented that layered $\mathrm{Cu}_{2} \mathrm{WS}_{4}$ can be converted from semiconducting to conductive nature by hydrogenation, along with enhanced electrical conductivity $\left(\sim 2.9 \times 10^{4} \mathrm{~S} \mathrm{~m}^{-1}\right.$ at room temperature $)$. The hydrogenation was carried out by a lithium intercalationassisted exfoliation approach in which the generation of intermediate precursor $\mathrm{Li}_{x} \mathrm{Cu}_{2} \mathrm{WS}_{4}$ played an important role in the exfoliation and hydrogen doping (Fig. 2d). During the hydrolysis reaction of $\mathrm{Li}_{x} \mathrm{Cu}_{2} \mathrm{WS}_{4}\left(\mathrm{Li}_{x} \mathrm{Cu}_{2} \mathrm{WS}_{4}+\mathrm{H}_{2} \mathrm{O} \rightarrow \mathrm{H}_{2} \uparrow+\mathrm{LiOH}+\right.$ $\mathrm{H}_{y} \mathrm{Cu}_{2} \mathrm{WS}_{4}$ ), the lithium atom was replaced with a hydrogen atom, resulting in improved conductivity of the materials. The free standing $\mathrm{Cu}_{2} \mathrm{WS}_{4}$ nanosheets are single-crystalline, and have a size of a few hundred nanometers with thickness of $\sim 1.0 \mathrm{~nm}$ (Fig. $2 \mathrm{e}-\mathrm{g}$ ), supportive to improving the electrochemical activity.

Besides these advantages of conventional nanostructured BMCs as discussed above, these nanostructures also have some disadvantages which cannot be ignored. Typically these nanostructures may agglomerate during the electrochemical process and suffer from large volume variation, resulting in poor electrochemical performance. Moreover, high surface area enhances the unwanted side reactions at electrode/electrolyte interfaces, resulting in thick SEI layer. Additional drawbacks such as low packing density and obvious self-discharge, result in lower volumetric energy density.

\section{$3 D$ hierarchical porous/hollow nanostructured BMCs}

The hollow nanostructures consist of nanoscale building blocks (such as nanosheets, nanorods) with hierarchical porous and hollow structures. They possess larger surface area and higher capacities compared with their solid counterparts, and offer unique properties such as huge interior hollow voids that can effectively accommodate the volume expansion. In addition, their high surface-to-volume ratio provides short diffusion pathways for mass/charge and leads to high rate capability of the electrodes. In the past few years, several nanostructured BMCs with diverse shapes, including ball-in-ball [55], hollow nanoboxes [56], hollow mesoporous microdumbbells [57], nanorodassembled hierarchical hollow nanostructures [58], nano-polyhedrons [59], core/shell nanospheres [60-67], yolk-shell nanocolloids [68], multi-shell hollow spheres [69,70], hollow nanoprisms [71], porous nanotubes [72-74], onion-like particles [75], capsule-like porous hollow structure [76], hollow tubular structures [77], hollow nanosheet arrays [78], and hollow nanocages $[79,80]$, have been extensively fabricated and used as electrode materials for high-performance EES devices. These hollow nanostructured BMCs have been synthesized by three approaches: soft templating, hard templating, and template-free approaches. In the templating approach, soft/hard templates are used as starting materials, then a thin layer of precursor materials is deposited onto these templates (e.g., silica spheres, polyacrylonitrile (PAN) polymer, polystyrene (PS) sphere, $\mathrm{Cu}_{2} \mathrm{O}$ ) by chemical solution precipitation or solvothermal approaches in combination with sulfurization/selenization, and subsequently, the templates are removed by chemical etching or calcination. The templates are usually removed by dissolution in acid/base, solvents like $\mathrm{N}, \mathrm{N}$-dimethylformamide (DMF), or calcination in air. Template-based nanostructured BMCs have been attractive due to their extraordinary benefits such as controlling the shape, size and structure. For instance, 1D $\mathrm{M}_{x} \mathrm{Co}_{3-x} \mathrm{~S}_{4}(\mathrm{M}=\mathrm{Ni}, \mathrm{Mn}, \mathrm{Zn})$ hollow tubular structure was prepared using soft polymeric nanofibers (NFs) as a soft template combining with designed procedures (Fig. 3a-g), in which the presence of functional groups in PAN NFs assisted in the deposition of precursor [77]. However, hard/soft template-based approaches always needed an extra time to remove the template which is a quite challenging process as well and requires toxic/ corrosive chemicals. Besides, the low yields typically for this type is another issue. Further, the hollow nanostructure has simple configuration and in some cases the surfaces of templates are not compatible with wanted/desired materials. In this regard, template-free approaches may be more attractive because these eliminate an additional step, reduce the production cost, and have simple fabrication procedures and better controlled size. Therefore, template-free approaches have been extensively applied to prepare the hollow nanostructures of BMCs. For instance, a two-step solvothermal approach was developed to synthesize well-architectured hollow $\mathrm{NiCoSe}_{2}$ (NCSe) submicrospheres, in which $\mathrm{NiCl}_{2} \cdot 6 \mathrm{H}_{2} \mathrm{O}, \mathrm{Co}\left(\mathrm{CH}_{3} \mathrm{COOH}\right)_{2} \cdot 4 \mathrm{H}_{2} \mathrm{O}$, and $\mathrm{SeO}_{2}$ were used as $\mathrm{Ni}, \mathrm{Co}$, and Se sources, respectively [81]. Using a similar two-step hydrothermal process, a ball-in-ball structure of $\mathrm{NiCo}_{2} \mathrm{~S}_{4}$ was prepared, in which the thickness of interiors of the as-synthesized $\mathrm{NiCo}_{2} \mathrm{~S}_{4}$ hollow spheres could be tuned by simply changing the reaction temperature during the anion exchange mechanism [55]. Three stages were involved during the ion exchange process: first, the solid sphere converted into core-shell $(0.2 \mathrm{~h})$, then yolk shell $(2 \mathrm{~h})$ and finally into ballin-ball nanostructures $(6 \mathrm{~h})$ as shown in Fig. $3 \mathrm{~h}-\mathrm{p}$.

The 3D porous hierarchical nanostructured BMCs consist of nanoscale building blocks (such as nanorods, nanowires, nanoflakes, and nanosheets) and hierarchical porous structures, but without hollow interior voids as observed in hollow structures. These hierarchical porous structures represent the properties of both nanoscale building blocks and as well micrometer-sized architectures. Moreover, these structures possess high accessible surface area and interconnected network, and can hold the electrolyte in mesopores present in these nanostructures. By means of various synthetic approaches, 3D hierarchical nanostructures, such as sea-urchin-like [82,83] flower-shaped [8487] hydrangea-like [88], nanosheet arrays [89,90], nanourchins [91], microaggregates [92,93], and pompen [94] have been 

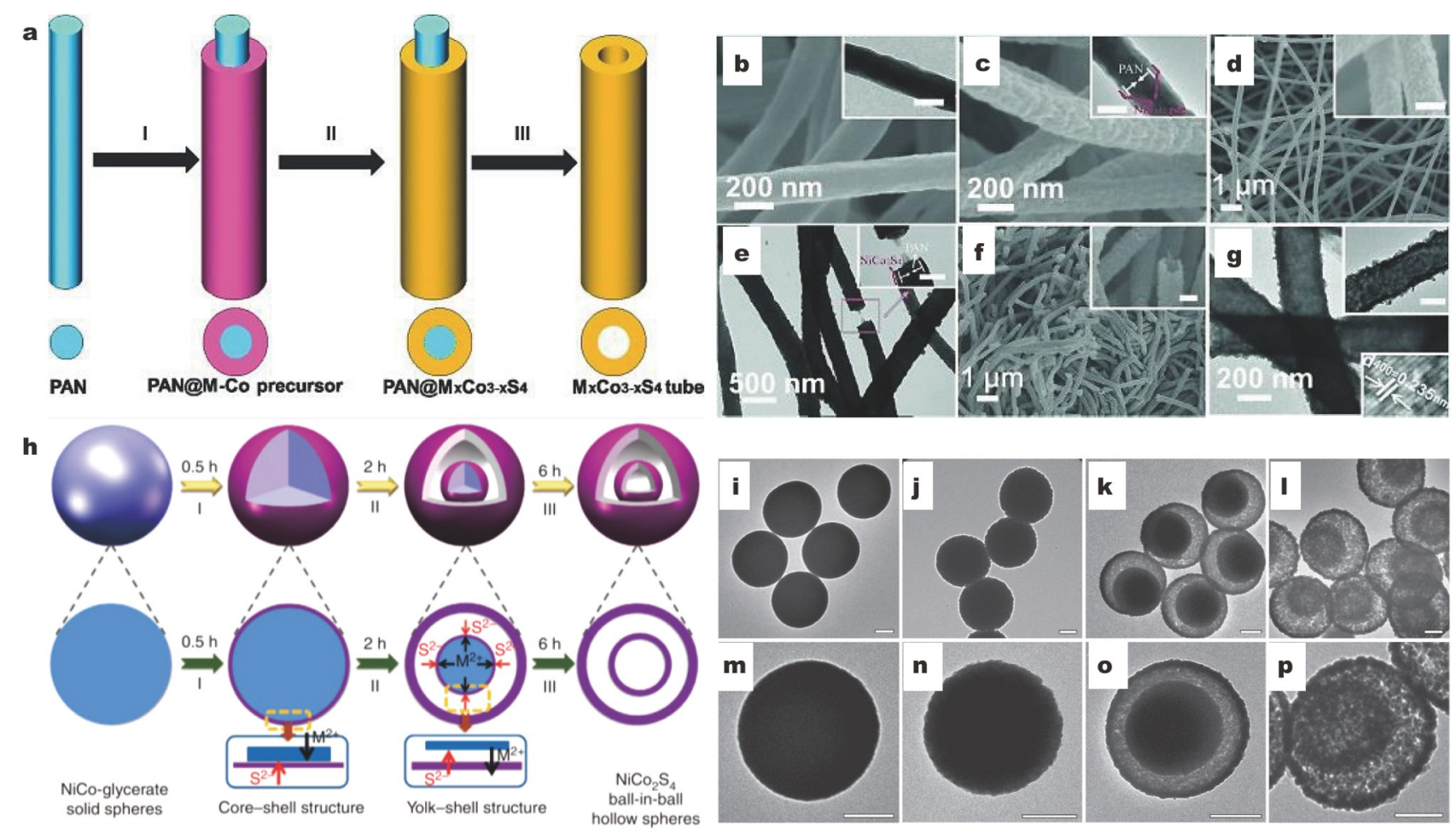

Figure 3 Formation mechanism of various hollow structures: (a-g) $\mathrm{M}_{x} \mathrm{Co}_{3-x} \mathrm{~S}_{4}$ nanotubes. (a) Schematic description of the formation of $\mathrm{M}_{x} \mathrm{Co}{ }_{3-x} \mathrm{~S}_{4}$ nanotubes. (b-g) Field emission scanning electron microscopy (FESEM) and TEM images of (b) PAN NFs, (c) the core-shell PAN@NiCo 2 precursor, (d, e) core-shell PAN@ $\mathrm{NiCo}_{2} \mathrm{~S}_{4}$ composite NFs, and (f, g) $\mathrm{NiCo}_{2} \mathrm{~S}_{4}$ hollow tubular structure. Scale bars in insets of (b-g) are 200 nm. Reprinted with permission from Ref. [77]. Copyright 2015, John Wiley and Sons. (h-p) $\mathrm{NiCo}_{2} \mathrm{~S}_{4}$ ball-in-ball hollow spheres. (h) Schematic illustration of the formation process of $\mathrm{NiCo}_{2} \mathrm{~S}_{4}$ ball-in-ball hollow spheres. Stage I, surface $\mathrm{NiCo}_{2} \mathrm{~S}_{4}$ formed by the anion exchange method. Stage II, further diffusion of $\mathrm{S}^{2-}$ and formation of $\mathrm{NiCo}_{2} \mathrm{~S}_{4}$ on the inner NiCo-glycerate core. Stage III, completion of the anion exchange reaction. $\mathrm{M}^{2+}$ refers to metal cations including $\mathrm{Ni}^{2+}$ and $\mathrm{Co}^{2+}$ ions. (i-p) FESEM and TEM images of samples. (i, m) NiCo-glycerate solid spheres and products obtained after sulfidation of NiCo-glycerate solid spheres at $160^{\circ} \mathrm{C}$ for different durations: (j, n) 0.5 h; (k, o) 2 h; (l, p) 6 h. Scale bars, 200 nm. Reprinted with permission from Ref. [55]. Copyright 2015, Nature Publishing Group.

realized. For instance, nano-flower like few-layered (2-3) Co-Sn$\mathrm{S}$ (FCoSnS) with a broad interlayer distance $(\sim 0.85 \mathrm{~nm})$ was reported, in which first $\operatorname{CoSn}(\mathrm{OH})_{6}$ precursor was synthesized by a co-precipitation reaction, which subsequently converted into nano-flower like FCoSnS by a controllable solvothermal process using thioacetamide (TAA) as the sulfidation agent [85]. This unique structure engineering design synergistically combines the functionalities of large interlayer spacing, effects of few layers, and hierarchical structure, resulting in fast electrochemical kinetics. In case of $\mathrm{NiCo}_{2} \mathrm{~S}_{4}$, it was exhibited that the supersaturation of nucleation and crystal growth was measured by the solvent polarity and solubility that can accurately control the morphology of $\mathrm{NiCo}_{2} \mathrm{~S}_{4}$ [95]. Among various morphologies, tube-like $\mathrm{NiCo}_{2} \mathrm{~S}_{4}$ microstructures showed the highest specific capacitance and better cycling stability compared with that of urchin-, cubic-, and flower-like morphologies, which may be ascribed to the scattered tube structure, and the promoted effective electrochemical reaction for active materials and electrolyte [95].

In summary, several effective synthesis approaches have been made for the preparation of nanostructured BMCs electrode materials. The produced BMCs showed diverse morphologies, such as nanoparticles, nanowires, nanorods, nanodots, nanosheets, microspheres, flower/cube-like structures, and diverse hollow and hierarchical structures, which intensely rely on the fabrication routes and different conditions used. Though different approaches have been used to synthesize BMCs, the growth mechanisms of BMCs with various compositions and morphologies are still not clear. Therefore, more investigations are still required to understand the underlying mechanisms.

\section{BMC-based hybrid nanostructures}

Hybridization is a fascinating approach to improve the shortcomings of individual counterparts and provide the advantages of synergistic effect. These composite nanoarchitectures not only enhance the structural stability and electronic conductivity but also boost the electrochemical performances. Besides in a hybrid structure, a common boundary is generated between two dissimilar components due to intimate contact, which is called interface. The generation of interfaces in the hybrid structure provides fascinating functionalities and substantial characteristics. Specifically, the generation of heterointerfaces at the atomic level in nanohybrids enables interfacial band alignment, surface modification, high specific surface area, charge transfer between different components and delocalization, and hence enhance the redox sites for ion storage, and expose more catalytic active sites [96]. Moreover, large interfacial area and tunable bandgap reduce the charge diffusion barrier, facilitate mass transport, improve the electronic conductivity, and stimulate the electrochemical properties for EES devices [96,97]. For example, 
the core-shell hybrid structure generates abundant interfaces at the core and shell boundary, which can greatly increase the charge transfer from the core to shell and synergistically enhance the electrochemical activity. Thus far, several materials including carbonaceous materials, Ni-F, and polymers have been revealed to hybridize with BMCs to produce functional composite architectures. This section will introduce and discuss the strategies and synthetic approaches for the fabrication of various kinds of BMC-based hybrid nanostructures.

\section{Hybridization of BMCs with carbon}

The most attractive and commonly used materials for the hybridization of BMCs to generate hierarchical structures are carbonaceous materials since carbon offers good electronic conductivity, large surface area, and high chemical stability. The higher electrical conductivities of carbonaceous nanomaterials provide effective electron transport pathways to composite architectures. Their high surface area and chemical stability provide structural stability, accommodate the large volume variations during repeated electrochemical cycling and as well prevent the agglomeration of BMCs. Bearing this in mind, BMCs have been composited onto, in or with diversified carbonaceous nanostructures, such as pyrolytic carbon, 1D CNT/CNF (carbon nanofiber), 2D graphene, $3 \mathrm{D}$ carbon clothes (CC), and hydrogels for improved electrochemical performances.

(I) Hybridization with pyrolytic carbon

Pyrolytic carbon has been composited with BMCs in three ways: (1) direct decoration of BMCs on carbon, (2) direct coating of carbon on the BMCs, and (3) in-situ impeding of BMCs into amorphous carbon matrix during the synthesis process of BMCs. In the first approach, only a few reports are available in which BMCs are anchored on carbon nanospheres or nanorods $[98,99]$. For instance, a core-shell heterostructure was generated in which $(\mathrm{Ni}, \mathrm{Co})_{0.85} \mathrm{Se}$ nanoparticles were uniformly decorated on carbon microspheres by a simple solvothermal process [98]. The main drawback of this approach is the agglomeration or detachment of the active material from the carbon template upon long electrochemical cycling due to direct contact of active materials with electrolytes. Therefore, direct coating of carbon on BMCs may be a more efficient way to improve the cycling performance. The pyrolysis of various carbon sources such as polypyrrole, glucose poly-dopamine (PDA), citric acid, bovine albumin (BSA) water-solution, and oleylamine were used to produce carbon-coated or nitrogen-doped carbon (NC)-coated BMCs. For example, NC-coated $\mathrm{NiCo}_{2} \mathrm{Se}_{4}$ $\left(\mathrm{NC@NiCo} \mathrm{Se}_{4}\right.$ ) with the morphology of 3D pomegranate-like porous spheres was synthesized by Liu et al. [100]. Herein the $\mathrm{Ni}$-Co-glycerol precursor (NCGP) was first prepared and then converted to $\mathrm{NiCo}_{2} \mathrm{Se}_{4}$ by a selenization process. During the selenization process, adequate anion exchange reactions happened between the outward diffusion of faster transition metal ions and the inward diffusion of $\mathrm{Se}^{2-}$ ions. In the final stage, BSA was decorated on $\mathrm{NiCo}_{2} \mathrm{Se}_{4}$ spheres, producing the BSA@Ni$\mathrm{Co}_{2} \mathrm{Se}_{4}$ intermediate product, which was further converted into $\mathrm{NC@NiCo} \mathrm{Se}_{4}$ after heating treatment. Besides the pomegranate-like porous spheres, other morphologies of BMCs such as solid nanoparticles [101], hollow spheres [102], yolk-shell polyhedral [103], multi-shelled hollow microspheres [104], nanoboxes [105], and nano crisp [106] have also been coated with carbon. Although, direct coating of carbon on BMCs is an effective strategy to improve the electrochemical performance, but the carbon layer may rupture during the electrochemical process and may cause poor cyclic stability. Therefore, it is anticipated that instead of direct carbon coating, the imbeding of $\mathrm{BMC}$ nanostructures into carbon or $\mathrm{NC}$ could be a more effective approach to improve the device performance. In this regard, lots of studies have been reported, in which various nanostructures of BMCs are embedded or distributed in carbon matrix [107-114].

Owing to their diverse structures and tunable compositions, ZIF-67 has been extensively used as precursor to synthesize BMCs embedded into carbon matrix [107,109-113]. The BMCs derived from ZIF-67 can deliver a large surface area, hierarchical porosities, and shortened diffusion path for ions. Moreover, the thermal treatment or pyrolysis of heteroatom-containing (nitrogen or sulfur) ligand can produce heteroatom-doped carbon, leading to higher conductivity of the composites and more electrochemical active sites. For example, the porous $\mathrm{Co}_{8} \mathrm{FeS}_{8} / \mathrm{N}$ $\mathrm{C}$ dodecahedral nanocages were fabricated by employing ZIF-67 as a template followed by pyrolysis and sulfuration routes, in which $\mathrm{Co}_{8} \mathrm{FeS}_{8}$ nanoparticles were embedded into $\mathrm{N}$-doped carbon matrix [113]. Recently, hollow mesoporous carbon spheres (HMCSs) as templates have been proposed for the impregnation/infiltration method to obtain BMCs/carbon hybrids [115,116]. Yang et al. [115] proposed a novel impregnation approach for the fabrication of ultrafine cobalt-iron selenides embedded in HMCSs. In this strategy, HMCSs acting as substrates were first prepared by the templating process that produced several mesopores in the shell and a huge void space (Fig. 4a). Then, two metal precursors (Co and Fe) were loaded into HMCSs by a repeated "drop and drying" route, subsequently selenization at different temperatures, resulting in Co$\mathrm{Fe}-\mathrm{Se}$ embedded in HMCSs (CFSe/C) (Fig. 4a-g). This strategy can not only restrain the overgrowth of BMCs during the thermal process, but also accurately control the structure and chemical composition of multiple elements. Specifically, various nanostructured CFSe/C hybrids varying from hollow (250 and $300^{\circ} \mathrm{C}$ ) to frog egg-like structures were obtained by simply controlling the selenization temperature that can be ascribed to the Ostwald ripening process (Fig. $4 \mathrm{a}-\mathrm{g}$ ).

(II) Synthesis of hierarchical nanohybrids of BMCs with 1D/ 2D carbon allotropes

Some of the striking characteristics of nanostructured BMCs are benefiting from their high surface area and plenty of electrochemically active sites. Nevertheless, the agglomeration of nanostructured BMCs during the electrochemical process reduces the effective surface area and redox sites. These challenges along with low electronic conductivity and large volume variation issues can be reduced to some extent by making homogeneous hybrid structures with conductive and flexible substrates at the nanoscale. Carbonaceous allotropes including graphene, CNTs, and CNFs have high mechanical strength, flexibility, and high surface area, which make them attractive templates for the growth of active electrode materials. These carbon allotropes also act as a stress buffer during the volume change process of BMCs and therefore improve the chemical and mechanical stability of the composite structure. Among them, graphene is the most commonly used substrate to make such nanohybrids. It is believed that graphene is the most electrical and thermal conductive form of carbon with outstanding mechanical properties and offers an abundant surface for the dispersion of active materials (BMCs) along with rapid 
a

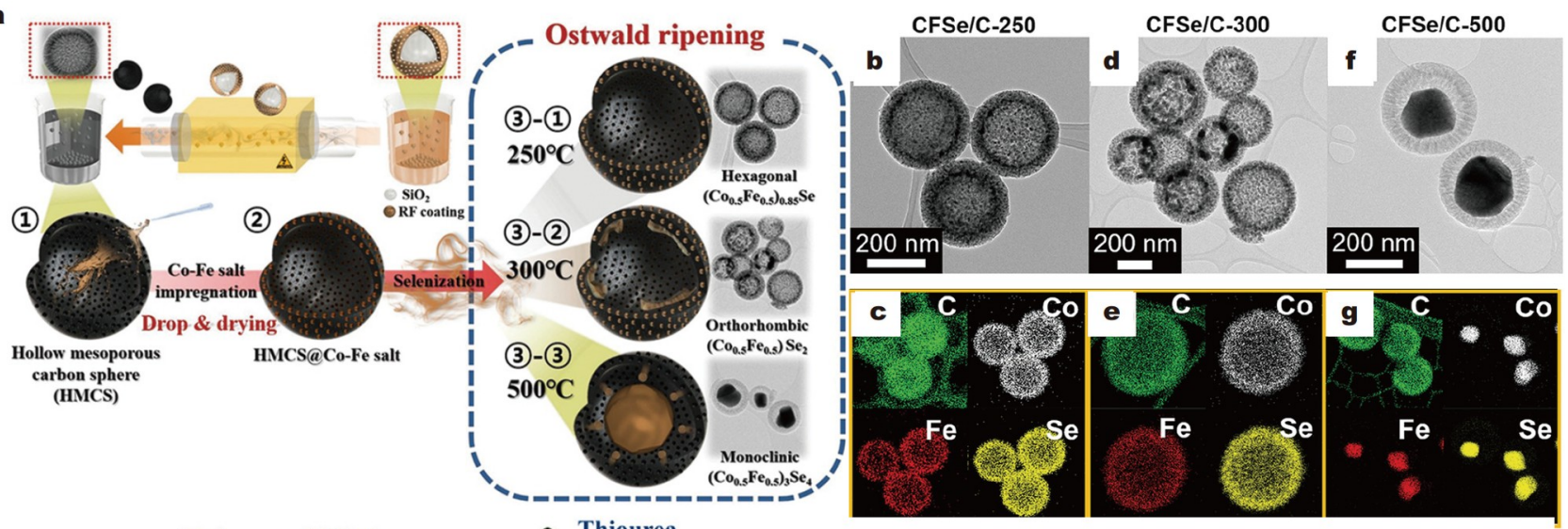

h

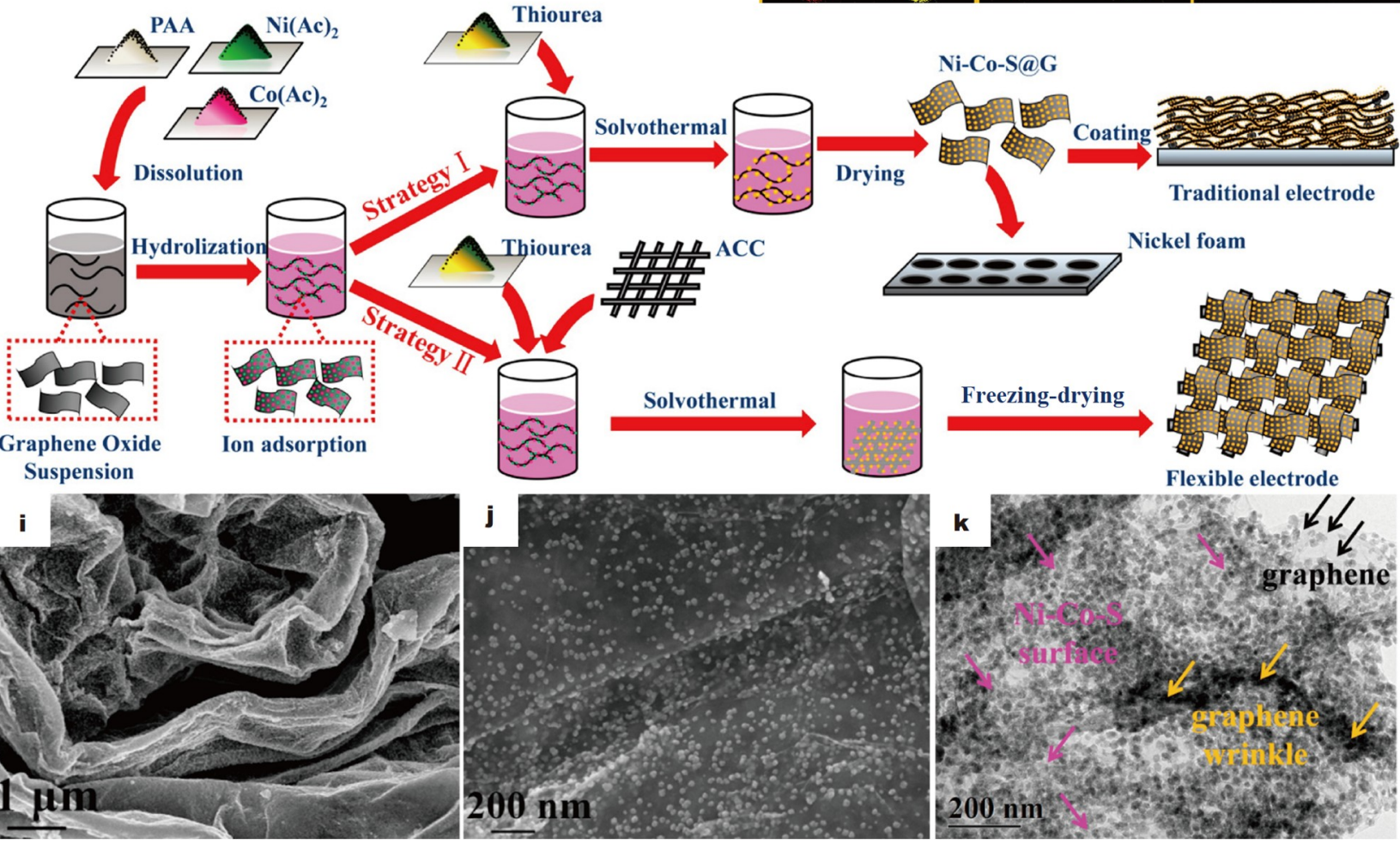

Figure 4 Hybrids of BMCs with various carbonaceous materials: (a-g) hybrids of Co-Fe-Se with HMCNs. (a) Schematic illustration of the formation of unique nanostructured Co-Fe-Se embedded in HMCNs. (b-g) Morphologies and elemental mapping images: (b) TEM, and (c) elemental mapping of CFSe/C250; (d) TEM, and (e) elemental mapping of CFSe-C-300; and (f) TEM, and (g) elemental mapping of CFSe/C-500. Reprinted with permission from Ref. [115]. Copyright 2020, John Wiley and Sons. (h-k) Hybrids of Ni-Co-S with graphene: (h) schematic description of the solvothermal process to fabricate Ni-Co-S@G for the traditional electrode (Strategy I) and Ni-Co-S@G/ACC (ACC: active cabon fiber cloth) flexible electrode (Strategy II) by employing ACC as the substrate. (i, j) Typical SEM images and (k) TEM image of Ni-Co-S@rGO with different magnifications. Reprinted with permission from Ref. [135]. Copyright 2017, Elsevier.

electron conduction and ion diffusion [117]. Moreover, the doping of non-metallic heteroatoms $(\mathrm{N}, \mathrm{P}$, or $\mathrm{S}$, etc.) into graphene can further effectively tune the electronic and chemical activity. Compared with single heteroatom doping, dual-doped graphene with two different heteroatoms is more effective in terms of high electrical conductivity, ionic diffusivity, structural stability, and suppressing the aggregation of graphene nanosheets. Besides, chemically converted graphene has abundant surface functional groups such as oxygen-containing groups particularly, and carboxylic acid groups (-COOH), which function as nucleation sites for active materials and make ease growth of other nanostructures on their surface. Several research groups have presented the fabrication or combination of BMCs on or with graphene to generate BMC-graphene nanohybrids
[118-131]. Solvothermal, hydrothermal, spray pyrolysis [124], along with solid-state sulfurization/selenization are the most common approaches for the fabrication of BMC-graphene nanohybrids $[125,126,131]$. The first BMC-graphene nanohybrid was reported by Peng et al. [125]. They presented a onestep hydrothermal process to grow ultrathin $\mathrm{NiCo}_{2} \mathrm{~S}_{4}$ nanosheets on reduced graphene oxide ( $\mathrm{rGO}$ ) to form a sheet-on-sheet hybrid nanostructure in a mixed solution of water and ethylenediamine (En) at $200^{\circ} \mathrm{C}$ using $\mathrm{Ni}\left(\mathrm{NO}_{3}\right)_{2} \cdot 6 \mathrm{H}_{2} \mathrm{O}$ and $\mathrm{Co}\left(\mathrm{NO}_{3}\right)_{2} \cdot 6 \mathrm{H}_{2} \mathrm{O}$ as precursors. Later on, many other BMCs with various morphologies including nanopartilces [128-131] nanosheets [118,127], nanorods [119], nanowires [120], platelike nanocrystals [124], nanodots [132], and cubes [133] have been composited with pure graphene or nitrogen/sulfur co- 
doped graphene. Although a lot of BMC-graphene compositebased investigations have been reported by several groups for high electrochemical performance, the bonding between graphene and BMCs is weak to immobilize BMCs onto the graphene sheets, leading to easy detachment of BMCs from conductive graphene matrix, which results in fast capacity fade and poor cycling stability. Moreover, it is also noted that the $-\mathrm{COOH}$ group is mainly distributed at the edge of $\mathrm{GO}$, and therefore homogeneous growth of BMCs is unsatisfactory or BMCs may anchor in the form of irregular bulk particles, leading to sluggish reaction kinetics and poor electrochemical performance. It has been reported that the strong chemical interactions between the chemically modified graphene and BMCs would be an effective way for homogenous growth of nanostructures on graphene and to improve the cyclic stability during repeated electrochemical cycling processes [134]. For instance, $\mathrm{FeSb}_{2} \mathrm{~S}_{4}$ was chemically anchored on amine-modified graphene $\left(\mathrm{FeSb}_{2} \mathrm{~S}_{4} / \mathrm{EN}-\mathrm{rGO}\right)$ with a porous interconnected sheet-like structure by the one-pot hydrothermal method [134]. Similarly, a surface-enriched Ni-Co-S@G hybrid was fabricated by the one-step solvothermal method, in which poly(acrylic acid) (PPA) with abundant - $\mathrm{COOH}$ functional groups was introduced during the synthesis process for well dispersion of Ni-Co-S nanoparticles on graphene (Fig. 4h-k) [135]. Such kinds of hierarchical nanostructures effectively mitigate the crumbling of the active material (BMCs) during the repeated charging/discharging process. Nevertheless, in most BMC-graphene nanocomposites, BMCs in the form of solid nanostructures (nanoparticles, nanorods, nanowires, etc.) have been decorated on the graphene. Recently, it has been reported that hollow nanostructured BMCs including hollow spheres [121] and hollow cubes [122,123], can also be anchored or encapsulated with graphene. This dual strategy may further synergistically improve the electrochemical performance. For example, a self-templated approach for the fabrication of graphene-wrapped nanoporous copper-cobalt selenide (rGO-CCSe) hollow spheres was developed [121]. During the selenization process, the hollow spheres of CCSe were generated due to the ion exchange process. Similarly, hollow $\mathrm{ZnSnS}_{3}$ hollow microcubes were encapsulated by nitrogen and sulfur co-doped graphene using a one-step hydrothermal process to enhance the sluggish reaction kinetics [122].

Besides, CNTs and CNFs have also been extensively utilized as matrices for the hybridization with BMCs to enhance the conductivity and generate more exposed active sites for better performance of EES devices [136-139]. Hydrothermal/solvothermal and microwave-assisted processes are the most common approaches for the synthesis of BMCs/CNTs hybrids. Unlike graphene, the pristine CNTs are hydrophobic, and therefore acid treatment is mostly required to generate the functional group that may provide abundant nucleation sites for the growth of BMCs. For instance, $\mathrm{Cu}_{2} \mathrm{SnSe}_{3}$ nanoparticles with a sphaleritelike structure were decorated on CNTs by a one-step hydrothermal method [137]. It was observed that the size of the original $\mathrm{Cu}_{2} \mathrm{SnSe}_{3}$ nanoparticles increased with the addition of CNTs, possibly due to that the CNTs offered limited growing sites for $\mathrm{Cu}_{2} \mathrm{SnSe}_{3}$ nanoparticles. However, the homogenous deposition of BMCs on the entire surface of CNTs is very critical, possibly due to insufficient adsorption energy between the BMCs and CNTs. For efficient use of BMCs, the uniform growth of BMCs on CNTs is very important. The electrodeposition technique could be an efficient approach for the uniform deposition of nanoparticles on various conductive substrates for preparing functional hybrids [140]. Recently, $\mathrm{CoNi}_{2} \mathrm{~S}_{4}$ nanoparticles with sizes of $10-15 \mathrm{~nm}$ were uniformly grown on CNTs using electrodeposition technique, in which CNT sponge was used as the working electrode, while $\mathrm{Ag} / \mathrm{AgCl}$ and platinum foil were served, respectively, as the reference and counter electrodes [141]. In all above BMCs-CNTs hybrids, the CNTs were used as a template for the growth of various BMCs. However, without using CNTs as the starting material, CNTs rooted in porous $\mathrm{Co}_{0.4} \mathrm{Zn}_{0.19} \mathrm{~S} @ \mathrm{~N}$ and $\mathrm{S}$ co-doped carbon dodecahedron were prepared by Li et al. [142]. They first designed the Co/Zn-based zeolitic imidazolate frameworks as the precursor to produce the intermediate product, $\mathrm{Co} / \mathrm{Co}_{3} \mathrm{ZnC@N-doped} \mathrm{carbon} \mathrm{dodecahe-}$ dron with rooted CNTs antennas using a chemical vapor reduction/deposition process, which was converted to Co- ZnS@N-S-C-CNTs by a sulfuration process. They reported that CNTs could not be generated in a pure $\mathrm{N}_{2}$ gas environment and so they used acetylene gas as a carbon source to produce the CNTs by the catalytic effect of Co metal catalyst [142].

Besides composites with binary components, ternary-component composites consisting of CNT/graphene or NC/graphene with BMCs have also been attained [143-147]. For example, an in-situ growth of $\mathrm{NiCo}_{2} \mathrm{~S}_{4}$ nanoparticles on $\mathrm{rGO} / \mathrm{CNT}$ was realized via a one-step hydrothermal method using $\mathrm{Ni}\left(\mathrm{NO}_{3}\right)_{2} \cdot 6 \mathrm{H}_{2} \mathrm{O}$, $\mathrm{Co}\left(\mathrm{NO}_{3}\right)_{2} \cdot 6 \mathrm{H}_{2} \mathrm{O}$, and $\mathrm{C}_{2} \mathrm{H}_{5} \mathrm{NS}$ as starting materials [143]. Recently, metal-organic frameworks (MOFs) have been extensively utilized as starting materials for the preparation of BMCs. By designing $\mathrm{Ni}$-Co-based MOFs as the precursor, a ternary hybrid consisting of porous $\mathrm{NC@NiCoSe}{ }_{4}$ microspheres, wrapped by graphene, was prepared by a solvothermal, carbonization, and selenization process [146].

(III) Synthesis of hierarchical nanohybrids of BMCs with 3D carbon templates

Compared with $1 \mathrm{D}$ or 2D carbon templates, 3D bulk carbon materials are very attractive and promising candidates for the growth of electrochemically active materials due to their high surface area, mechanical stability, well-defined pathways for electrolyte infiltration, and interconnected conductive network for the transport of both ions and electrons. 3D templates are featured with 1D CNTs or CNFs, 2D graphene nanosheets, or amorphous carbon and so preserve the intrinsic properties of carbonaceous allotropes. For example, 3D graphene architecture consisting of 2D graphene nanosheets provides multiple advantages in preventing the intense aggregation of individual graphene nanosheets, high effective surface area, and 3D channels for electron conduction and fast transfer of ions. Moreover, the extraordinary flexibility of these $3 \mathrm{D}$ carbon skeletons and the presence of voids mitigate the volume variations during the electrochemical process. These 3D carbon materials have been demonstrated to be attractive templates to assemble various nanostructured BMCs for making functional hybrids. For instance, hierarchical $\mathrm{NiCo}_{2} \mathrm{Se}_{4}$ with different morphologies (nanoneedles/nanosheets) were grown on the $\mathrm{N}$-doped $3 \mathrm{D}$ porous graphene skeleton by a solvothermal treatment followed by gas-phase selenization process, and the resultant hybrid can be used as a free standing electrode for EES [148]. Recently, a flexible graphene film was prepared by a vacuum filtration method and directly used to grow $\mathrm{FeCo}_{2} \mathrm{~S}_{4}$ array by a two-step hydrothermal method [149]. Besides, in-situ [150] and ex-situ $[151,152]$ growth of BMCs in graphene aerogel/hydrogel has also 
been reported. Using an in-situ growth strategy, Li et al. [150] synthesized $3 \mathrm{D} \mathrm{Ni}_{x} \mathrm{Co}_{1-} \mathrm{S}_{2}$ /graphene hydrogels via a one-step hydrothermal process using thiourea, $\mathrm{NiCl}_{2} \cdot 6 \mathrm{H}_{2} \mathrm{O}$, and $\mathrm{CoCl}_{2}$. $6 \mathrm{H}_{2} \mathrm{O}$ as precursors. The $\mathrm{Ni}_{x} \mathrm{Co}_{1-x} \mathrm{~S}_{2}$ particles were distributed homogenously on the $3 \mathrm{D}$ interconnected porous network of graphene nanosheets with pore sizes in the range from several to tens nanometers. Interestingly, with increasing $\mathrm{Ni}$ content, the particle size of $\mathrm{Ni}_{x} \mathrm{Co}_{1-} \mathrm{S}_{2}$ on the nanosheets changed from $50 \mathrm{~nm}$ to $1.5 \mu \mathrm{m}$ due to different electrostatic interactions of $\mathrm{Ni}^{2+}$ and $\mathrm{Co}^{2+}$ cations with negatively charged graphene nanosheets, in which $\mathrm{Ni}_{0.31} \mathrm{Co}_{0.69} \mathrm{~S}_{2}$ composition showed the best electrochemical results.

Recently, it was demonstrated that the CNT sponge developed by the chemical vapor deposition (CVD) method possesses macroscopic porosity, thermal stability, excellent flexibility, and robustness that have been reported for the compressible substrate [153-156]. Till now only a few studies are reported for the 3D CNT@BMCs hybrid sponge, in which $\mathrm{NiCo}_{2} \mathrm{~S}_{4}$ nanoparticles are uniformly anchored on the CNT sponge by the one-step hydrothermal method [141]. However, the CVD technique has been used for the preparation of most of the $3 \mathrm{D}$ carbon substrates such as graphene foam or CNT sponge, which is highcost and complex. In this regard, N-doped carbon foam (NCF) derived from direct carbonization of commercially available melamine foam can be effective that can accomplish a simultaneous improvement in the form factor flexibility, low cost, lightweight and facilitate the homogenous growth of active materials [157-159]. Few reports are available in which various morphologies of BMCs including nanoparticle micro-disks, nanotube arrays, or nanosheets were homogeneously dispersed in NCF using both in-situ and ex-situ strategies [157-160]. For instance, Chen et al. [157] used the in-situ strategy for uniform dispersion of $\mathrm{NiCo}_{2} \mathrm{~S}_{4}$ nanoparticles in NCF via a two-step method, in which first melamine resin was prepared, mixed with $\mathrm{Ni}$-Co aqueous solution, solidified to form hybrid melamine foam, and then annealed under an inert atmosphere with subsequent sulfurization.

Moreover, CC, carbon textiles (CT), or carbon fiber papers (CFP) as a template are popular due to their higher flexibility, rich porous structures, and pore volumes. Compared with graphene or CNT-based 3D bulk templates, extensive effort has been devoted to the deposition of various morphologies of BMCs including nanoparticles, nanowires, nanotubes, nanoflakes, and nanosheets on CC [161-166], CT [167], and CFP [168-171] via different methods including hydrothermal/solvothermal and electrodeposition. Note that hydrothermal is the most common and effective approach for the fabrication of CCbased BMCs hybrid. As a typical example, interconnected $\mathrm{CoNi}_{2} \mathrm{~S}_{4}$ nanosheets with different shapes and thicknesses were deposited on CC via a precursor transformation route by adjusting the anions in Ni-Co salts [165]. It was observed that the anion has a vital role in the formation of various morphologies, resulting from different growth kinetics during the crystal growth [165]. It was found that $\mathrm{CoNi}_{2} \mathrm{~S}_{4}$ nanosheets obtained with $\mathrm{NO}_{3}{ }^{-}$anion were ultrathin and displayed the highest electrochemical performance. MOFs have also been used as precursors to synthesize carbon-embedded BMCs. Using this strategy, hierarchical $\mathrm{Zn}_{x} \mathrm{Co}_{1-x} \mathrm{~S}$ (where $x=0,0.25,0.5,0.75$, and 1.0) nanoparticle-embedded in NC was directly anchored on a flexible CC by an in-situ synthesis strategy, and the best electrochemical performance was achieved by $\mathrm{Zn}_{0.76} \mathrm{Co}_{0.24} \mathrm{~S}$ composition [172]. The $\mathrm{Zn}_{x} \mathrm{Co}_{1-x} \mathrm{~S} / \mathrm{NC}$ was prepared by a solution method followed by calcination and a controlled sulfurization process of $\mathrm{Zn}$-Co oxides derived from MOF using TAA as the sulfur source (Fig. 5a). The morphology analysis revealed that the $\mathrm{Zn}$-Co oxides were in nanosheets form, and during the sulfurization process, the nanosheets were gradually converted to nanoparticles according to the Kirkendall effect (Fig. 5b-g).

\section{Hybridization of BMCs with metal foam}

Metal foams such as Ni-F are highly conductive and porous templates for the deposition of various electroactive materials to prepare the electrodes for EES devices. Compared with 3D bulk carbon-based templates, metallic foams exhibit better mechanical properties and higher electronic conductivity, but lower surface area and larger density [41]. Till now, a large variety of BMCs with different chemical compositions and morphologies have been grown on Ni-F templates using different methods [173-179]. As a typical example, $\mathrm{NiGa}_{2} \mathrm{~S}_{4}$ yolk-shell microspheres consisting of crumpled nanosheets anchored on $\mathrm{Ni}-\mathrm{F}$ were prepared through the most common hydrothermal and effective sulfurization process. During the hydrothermal process, the dissolved $\mathrm{Ni}^{2+}$ ions in the water solution chemically reacted with $\mathrm{Co}_{3}{ }^{2-}$ and $\mathrm{OH}^{-}$that were generated via a hydrolysis reaction of urea. The $\mathrm{NH}_{4} \mathrm{~F}$ was used as the activation solvent and served as a buffering agent to adjust the $\mathrm{pH}$ value of the solution [178]. During the sulfurization process, the NiGa-LDH (layered double hydroxide) precursor derived from the complex-oriented attachment process was converted into $\mathrm{NiGa}_{2} \mathrm{~S}_{4}$ yolk-shell microspheres based on the Kirkendall effect [178]. Besides, successive ionic layer adsorption reaction (SILAR) has been a simple approach for the preparation of different metal sulfides/ oxides nanostructures. Recently, this novel SILAR approach was also used for the growth of 3D hierarchical nickel molybdenum sulfide (NMS) nanoflakes on Ni-F to develop high performance electrode materials (Fig. 5h-1) [173]. The morphology, structure, and electrochemical performance of NMS could be affected by selective SILAR cycles (10, 20, 30, or 40 cycles). It was observed that the density of NMS nanostructure deposited on Ni-F is directly proportional to SILAR cycles and better performance was obtained with 40 SILAR cycles (Fig. 5h-1) [173].

In addition, the morphology and structure of BMCs can be tuned by controlling the concentration of the mixture of aqueous ionic liquid (IL) during the chemical solution method, and desired hierarchical hollow nanostructures can be achieved [177]. For instance, the growth of $1 \mathrm{D}$ hollow nanoneedles, 2D porous nanosheets, and $3 \mathrm{D}$ hollow nanoarrow arrays of $\mathrm{NiCo}_{2} \mathrm{~S}_{4}$ on Ni-F was achieved through controlling IL 1-octyl-3-methylimidazolium chloride $([\mathrm{OMIm}] \mathrm{Cl})$ concentration using a twostep solvothermal method in which [OMIm]Cl severed as both capping agent and solvent that controlled the growth direction of Ni-Co precursor arrays [177]. The better electrochemical properties were obtained with $3 \mathrm{D}$ hollow nanoarrow array compared with $1 \mathrm{D}$ hollow and $2 \mathrm{D}$ hierarchical porous structures due to its high specific surface area, robust structural stability, and unique porous hollow structure [177]. Besides chemical solution routes, the growth of BMCs on $\mathrm{Ni}-\mathrm{F}$ was also made using the electrodeposition method [174,180]. In addition, few studies are also reported in which carbonaceous material such as graphene was first deposited on $\mathrm{Ni}-\mathrm{F}$ and then combined with active materials (BMCs). In this way, the issues of pure $\mathrm{Ni}-\mathrm{F}$ such as lower specific surface area and larger density could be 

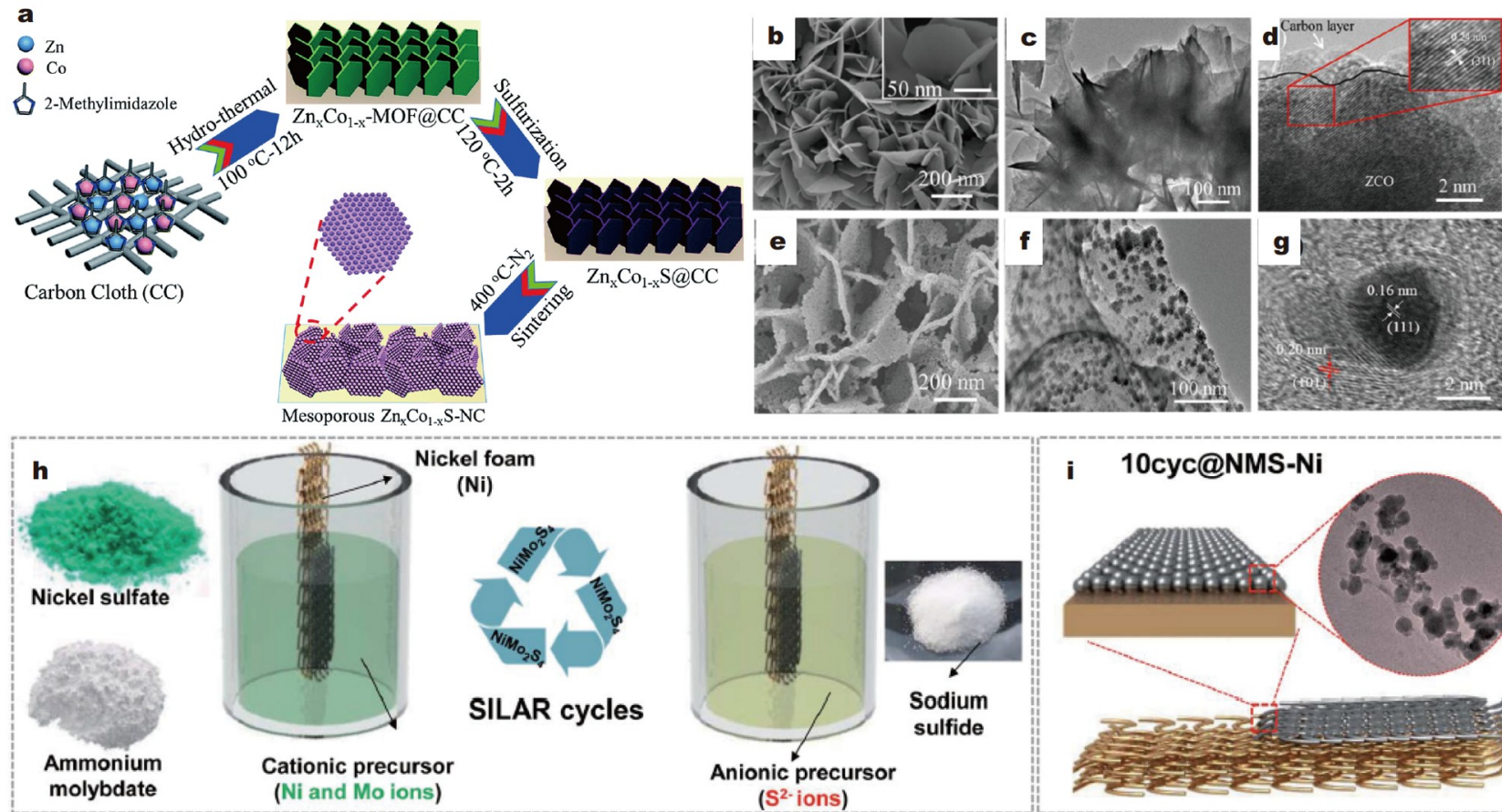

(Ni)
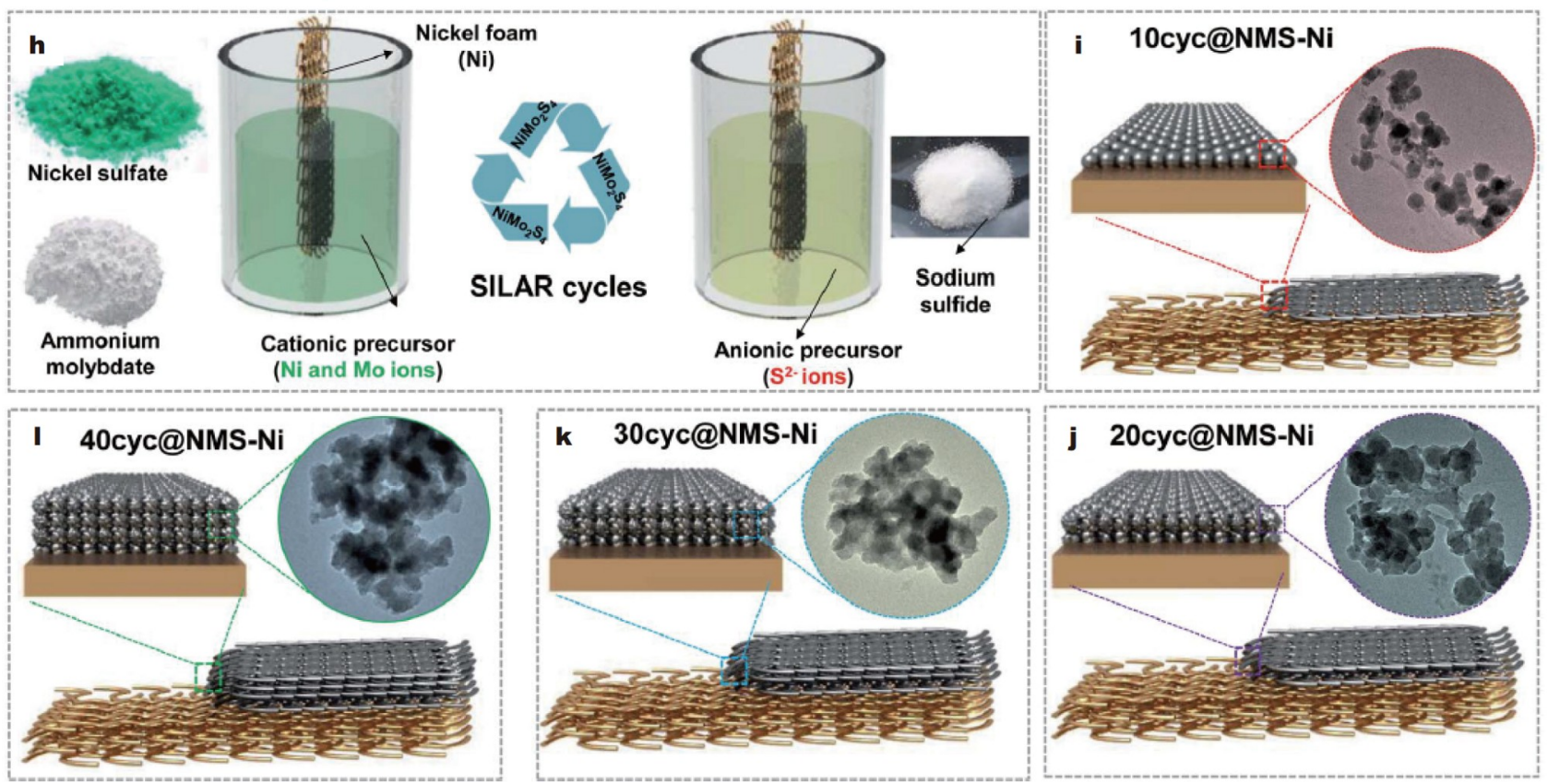

Figure 5 Hybrids of BMCs with various 3D templates: (a) schematic illustration of growing bimetallic MOF-derived nanosheets on a CC substrate and their conversion to mesoporous $\mathrm{Zn}_{x} \mathrm{Co}_{1-x} \mathrm{~S}$ nanoparticles embedded in $\mathrm{N}$-doped carbon. (b-g) Morphologies of $\mathrm{Zn}_{0.76} \mathrm{Co}_{0.24} \mathrm{O}_{4}-\mathrm{NC}$ and $\mathrm{Zn}_{0.76} \mathrm{Co}_{0.24} \mathrm{~S}-\mathrm{NC}-\mathrm{II}$ at the CC substrate. (b) SEM image of ZCO-NC, and (c, d) corresponding low and high-resolution TEM images. (e) SEM image of ZCS-NC-II, and (g, h) corresponding low and high-resolution TEM images. Reprinted with permission from Ref. [172]. Copyright 2019, Royal Society of Chemistry. (h-l) Schematic description of the facile SILAR growth of NMS on the Ni substrate. (h) Deposition process and chemical reactants used for SILAR, and (i) 10cyc@NMS-Ni, (j) 20cyc@NMS-Ni, (k) 30cyc@NMS-Ni and (l) 40cyc@NMS-Ni electrodes. Reprinted with permission from Ref. [173]. Copyright 2019, Royal Society of Chemistry.

improved to some extent. Moreover, seamlessly connected graphene networks prepared on Ni-F by CVD can obtain decent electronic conductivity and can be used as an attractive template to assemble various BMCs [179]. As a typical example, Zhao's group [179] fabricated the $\mathrm{NiCo}_{2.1} \mathrm{Se}_{3.3}$ nanosheets/3D-graphene/ Ni-F through CVD followed by a two-step hydrothermal process. The reported surface of $3 \mathrm{D}$-graphene/Ni-F was smooth with ripple-like wrinkles because of uniform growth of graphene on $\mathrm{Ni}-\mathrm{F}$, whereas $\mathrm{NiCo}_{2.1} \mathrm{Se}_{3.3}$ nanosheets array on the $3 \mathrm{D}$ graphene/Ni-F was uniform, dense and vertical on the surface of 3D-graphene/Ni-F, which can provide high electrochemically active surface area, shortened pathways for charge transport and high efficiency of energy storage [179].

Fabrication of hierarchical nanohybrids of BMCs with metal compounds

To further improve the electrochemical performance and optimize the structural properties of BMCs efficiently, diverse metal compounds including mono/binary metal oxides (hydroxides), mono/binary metal sulfides or selenides have also been composited with BMCs. Compared with single-component, BMCs compound composites present better electrochemical performance due to their unique properties, controlled structural degradation, full use of synergic effect of different metal ions, and the benefits of dissimilar active components [25]. In particular, heterogeneous junction structures between BMCs and metal compounds can provide more active sites, short diffusion path for electrolyte, expanded contact area at the electrode/ electrolyte interface, and fast charge and mass transfer kinetics. For instance, conductive core@shell $\mathrm{NiCoSe}_{2}(\mathrm{NCSe}) @ \mathrm{Co}_{9} \mathrm{Se}_{8}$ hollow nanospheres were prepared via a two-step solvothermal route in which $\mathrm{Co}_{9} \mathrm{Se}_{8}$ nanosheets were homogenously grown on a hollow NCSe core [181]. Similarly, various electrochemical active materials such as $\mathrm{SnS}_{2}$ [182], $\mathrm{CoS}_{2}$ [183], and $\mathrm{NiMoO}_{4}$ [184], have been combined with different BMCs to build heterostructured composites.

However, BMCs compound composites without any conductive substrates often have low electrical conductivity. More- 
over, large surface energy and low ferromagnetism in these nanostructured materials may lead to intense aggregation, causing reduced surface area and number of electroactive sites, resulting in poor performance [185]. To avoid these issues and fully utilize electrochemical active materials, BMCs compound composites with various nanoarchitectures such as heteronanoparticles [186], core-shell nanotubes [187,188], yolk-shell microspheres [189,190], nanosheets arrays [191], wire-like structure [192], and core-shell nanostructured arrays [193] have been directly grown on diverse conductive templates. As a representative example, a 3D macroporous lollipop-like $\mathrm{MnCo}_{2} \mathrm{~S}_{4} / \mathrm{FeCo}_{2} \mathrm{~S}_{4}$ heterostructure was deposited on a porous NCF via a two-step hydrothermal approach accompanied with an ion exchange process [194]. The hierarchical $\mathrm{MnCo}_{2} \mathrm{~S}_{4} /$ $\mathrm{FeCo}_{2} \mathrm{~S}_{4}$ lollipops consisting of porous nanosheet-built $\mathrm{MnCo}_{2} \mathrm{~S}_{4}$ spheres and rough $\mathrm{FeCo}_{2} \mathrm{~S}_{4}$ nanoneedles enhanced the electrochemical active sites and structural integrity. Similarly, a compound composite consisting of small core@shell $\mathrm{CoS}_{x} @ \mathrm{Cu}_{2} \mathrm{MoS}_{4}$ (CMS) structures was effectively and homogenously deposited on a $\mathrm{MoS}_{2} / \mathrm{N}, \mathrm{S}$ co-doped graphene heteronetwork ( $\mathrm{CoS}_{x} @ \mathrm{CMS}$ $\mathrm{MoS}_{2} / \mathrm{NSG}$ ) through a unique route (Fig. 6a-j)) [185]. The formation mechanism of $\mathrm{CoS}_{x} @ \mathrm{CMS}-\mathrm{MoS}_{2} / \mathrm{NSG}$ involved multiple steps. During the first step, $\mathrm{Co}$ (thiourea $)_{4}\left(\mathrm{NO}_{3}\right)_{2}$ com- plexes were generated in the presence of $\mathrm{Co}^{2+}$ cations and thiourea linkers, and these complexes severed as active substrates to adsorb $\mathrm{Cu}^{2+}$ on their surface. Meanwhile, $\mathrm{MoS}_{4}{ }^{2-}$ anions reacted with $\mathrm{Cu}^{2+}$ cations and produced a thin layer of CMS precipitates uniformly depositing around $\mathrm{Co}$ (thiourea) 4 $^{-}$ $\left(\mathrm{NO}_{3}\right)_{2}$ complexes. Finally, Co(thiourea $)_{4}\left(\mathrm{NO}_{3}\right)_{2} @ \mathrm{CMS}$ transformed into a core@shell $\mathrm{CoS}_{x} @ \mathrm{CMS}$ structure via a thermal treatment step. Moreover, during the annealing process at high temperatures, the unreacted $\left(\mathrm{NH}_{4}\right)_{2} \mathrm{MoS}_{4}$ converted into $\mathrm{MoS}_{2}$ nanosheets and evenly dispersed on N,S co-doped graphene (Fig. 6f-j). Besides hydrothermal/solvothermal or electrodeposition methods, currently an in-situ electrochemical activation approach was developed to make highly porous NCSe@NiOOH/CoOOH (NCSe@NCH) heterostructures decorated on $\mathrm{CC}$ as multifunctional electrode for battery applications [195]. First they deposited the porous NCSe nanosheets on CC via a two-step hydrothermal/solvothermal process and subsequently transformed them into porous NCSe@NCH heterostructures by an in-situ electrochemical phase transformation process [195]. During the phase transformation process, the structure of NCSe skeleton was well preserved along with the formation of abundant electroactive sites that are advantageous for both Faradaic and ORR/OER reactions.
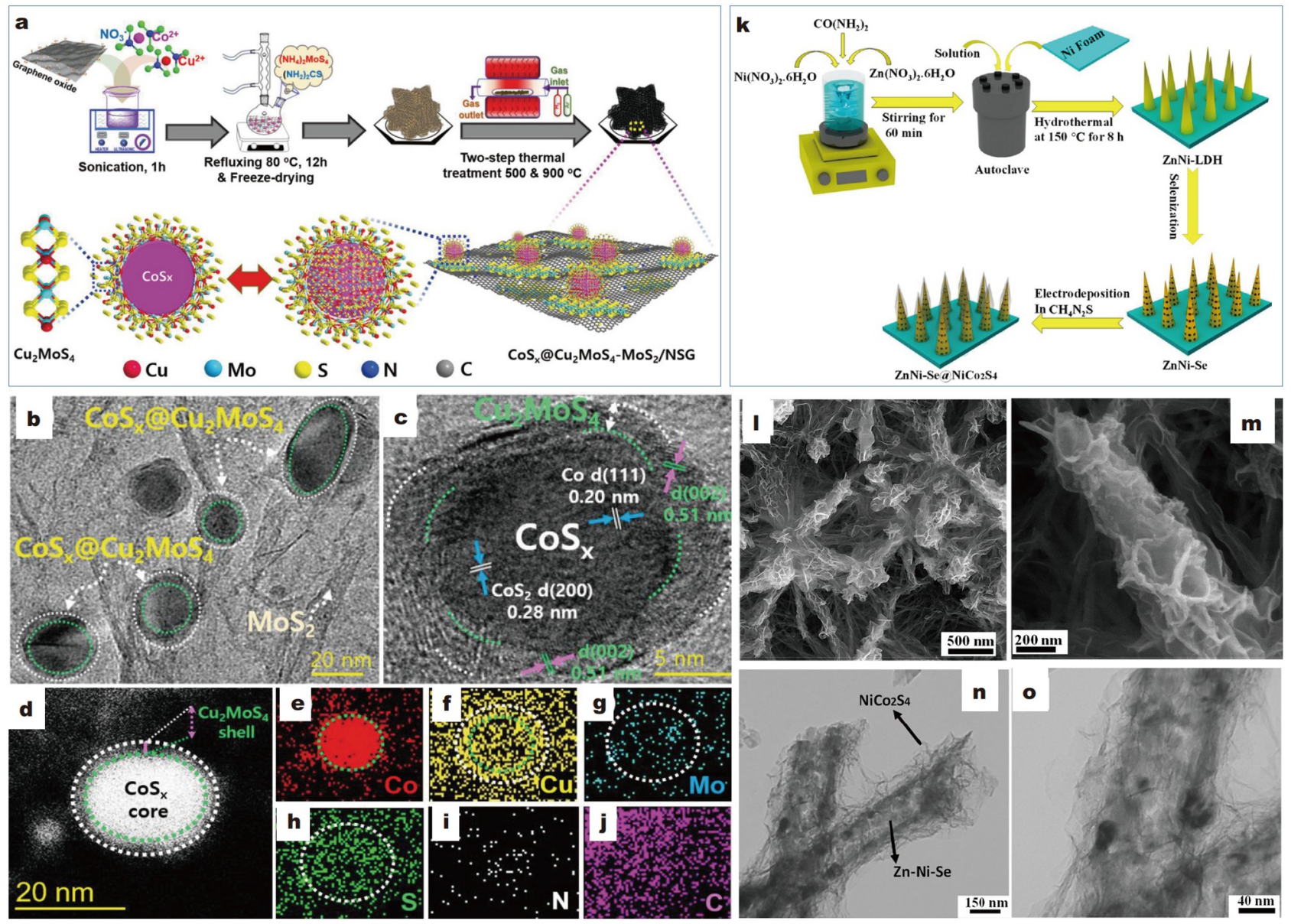

Figure 6 Formation mechanism of various heterostructured BMCs. (a) Schematic displaying for the fabrication of CoS $@$ @MS-MoS $2 /$ NSG hybrid. (b, c) HRTEM images of $\mathrm{CoS}_{x} @ \mathrm{CMS}-\mathrm{MoS}_{2} / \mathrm{NSG}$ hybrid. (d) STEM image of $\mathrm{CoS}_{x} @ \mathrm{CMS}-\mathrm{MoS}_{2} / \mathrm{NSG}$ and its EDS color mapping of (e) Co, (f) Cu, (g) Mo, (h) S, (i) N, and (j) C. Reprinted with permission from Ref. [185]. Copyright 2020, John Wiley and Sons. (k) Schematic showing for the formation processes of Zn-Ni-Se@NCS on the Ni-F. (l, m) SEM images of the Zn-Ni-Se@NCS@NF. (n, o) TEM images of the Zn-Ni-Se@NCS. Reprinted with permission from Ref. [199]. Copyright 2020, American Chemical Society. 
In addition to carbonaceous templates, BMCs compound composites have also been grown on highly conductive Ni-F. In this context, an extensive effort has been devoted to immobilizing the BMC compound composites on Ni-F [196-204]. As a typical example, hierarchical heterostructured $\mathrm{Zn}-\mathrm{Ni}-\mathrm{Se} @ \mathrm{NiCo}_{2}-$ $\mathrm{S}_{4}$ on the Ni-F surface was developed via two stages (Fig. 6k) [199]. Specifically, first $\mathrm{Zn}-\mathrm{Ni}$ nanowires were fabricated on the $\mathrm{Ni}-\mathrm{F}$ by a hydrothermal process and then converted into $\mathrm{Zn}-\mathrm{Ni}$ Se by a selenization process in accordance with the ion exchange mechanism. In the second stage, $\mathrm{NiCo}_{2} \mathrm{~S}_{4}$ (NCS) nanosheets were in-situ deposited on the $\mathrm{Zn}-\mathrm{Ni}$-Se core via an electrodeposition technique. It was revealed that the core $1 \mathrm{D} \mathrm{Zn}-\mathrm{Ni}-\mathrm{Se}$ hollow nanowires and the shell 2D NCS nanosheets are interconnected with each other and generate a $3 \mathrm{D}$ porous and hierarchical architecture, resulting in the effective and rapid conduction/transmission of electrons and ions (Fig. 61-o). Such kind of fabrication protocol can be further extended in the synthesis of other kinds of TMC electrodes with core-shell configuration to improve the electrochemical response.

\section{Heteroatom-doped BMCs}

In addition to morphological control and hybridization, heteroatom doping is another attractive strategy to strengthen electrochemical performance. Doping of a minor amount of foreign atoms (metal and metalloid elements) into BMCs may cause slight lattice modulation (such as expansion, contraction, or distortion) in crystal structures that can result in the formation of more defects and higher mobility. These crystal defects produce more existing active sites and reduce the strain during the electrochemical process. Besides, foreign atom doping can optimize the electronic properties of BMCs, and improve the electronic conductivity and ionic diffusivity by lowering the diffusion barrier. In addition, it can tune the local electronic environment of host atoms and alter the adsorption and desorption energy, consequently boosting catalytic performance [205]. The phosphorous (P) doping into $\mathrm{NiCo}_{2} \mathrm{~S}_{4}$ is an ideal strategy to tune the structural and electrochemical properties, as physiochemical properties (such as electronegativity and atomic radius) of $\mathrm{P}$ atoms are nearly analogous to that of sulfur (S) or selenide (Se) atoms. For the illustration, P-doped $\mathrm{NiCo}_{2} \mathrm{~S}_{4}$ was reported by phosphidation to reveal enhanced electrical conductivity and more diffusional channels due to the enlarged charge carriers and crystal defects, resulting in improved capacitive performance [206]. However, in most cases the fabrication process for both sulfidation/selenization and doping is timeconsuming and complicated as multi steps are involved during the synthesis process and may cause unwanted morphology and disordered interfaces or ruin the compound structure [18,206,207]. Therefore, a one-step synthesis process (i.e., phospho-sulfidation/phospho-selenization) could be an attractive strategy owing to lower cost, easier and less time-consuming procedures, and requisite for large-scale production than that of complicated multi-step fabrication processes [208,209]. Recently, Lim et al. [205] used only one bifunctional precursor $\left(\mathrm{P}_{2} \mathrm{~S}_{5}\right)$ for phosphosulfidation and developed 3D hierarchical flower-like P-doped Ni-Fe disulfide (P-doped Ni-Fe-S) microspheres. They used metal chlorides, urea and $\mathrm{NH}_{4} \mathrm{~F}$ as starting materials. The urea helped in the formation of the $\mathrm{LDH}$ structure by releasing $\mathrm{OH}^{-}$and $\mathrm{CO}_{3}{ }^{2-}$ in the fabrication process. $\mathrm{NH}_{4} \mathrm{~F}$ controlled the morphology of the flower-like morphology, and the $3 \mathrm{D}$ flower-like morphology remained preserved after the phosphosulfidation process. In addition to P doping into BMCs, S or Se atoms have also been doped into BMCs [210,211]. However, the studies on the fabrication of S or Se-doped BMCs for energy storage are quite limited. Lin et al. [210] reported the synthesis of free-standing Se-doped nickel-cobalt sulfide (Se$\mathrm{NiCoS}$ ) electrode with controllable components using hydrothermal and sulfuration/selenization processes. The appropriate $\mathrm{Se}$ doping in Ni-Co-S significantly enhanced the electrochemically active surface area and decreased the charge transfer resistance. Moreover, the role of Se dopants was examined using $\mathrm{X}$-ray photoelectron spectroscopy (XPS) and TEM analysis in which it was concluded that Se dopants were changed into $\mathrm{SeO}_{x}$, and electrochemical-generated oxyhydroxides $(\mathrm{NiOOH}$ and $\mathrm{CoOOH}$ ) were mainly involved in electrochemical processes [210].

Recently, metal atoms $\mathrm{M}(\mathrm{M}=\mathrm{Fe}, \mathrm{Mn}, \mathrm{Cu}, \mathrm{Zn})$ doped into simple BMCs and compound BMCs have also been explored to tune the energy storage properties [212-215]. For instance, hierarchical MOF-derived $\mathrm{Cu}$-doped $(\mathrm{Co}-\mathrm{Ni})_{2} \mathrm{~S}_{4}$ nanotubes were prepared by an in-situ strategy of sulfurization followed by synchronous etching and doping [216]. Upon sulfurization at high temperatures, simultaneously multiple ions $\mathrm{Cu}^{2+}, \mathrm{Co}^{2+}, \mathrm{Ni}^{2+}$ reacted with $\mathrm{S}^{2-}$, and generated ternary chalcogenides (i.e., $\left.\mathrm{Cu}(\mathrm{Co}-\mathrm{Ni})_{2} \mathrm{~S}_{4}\right)$ with a hollow structure in which $\mathrm{Cu}$ doping enhanced the electrochemical conductivity, resulting in enhanced performance. However, unraveling the effect of metal doping on the electrochemical performance of BMCs has been remained a big challenge. Lai et al. [217] introduced Fe doping domains on the surface of $\mathrm{NiCo}_{2} \mathrm{~S}_{4}\left(\mathrm{Fe}-\mathrm{NiCo}_{2} \mathrm{~S}_{4}\right)$ nanosheets and wrapped on the N,S-doped ordered mesoporous carbon $\left(\mathrm{Fe}-\mathrm{NiCo}_{2} \mathrm{~S}_{4} @ \mathrm{~N}, \mathrm{~S}-\mathrm{CMK}\right)$ for boosted performance (Fig. 7a-f). Further, they demonstrated the effect of Fe doping on the electronic structure of $\mathrm{NiCo}_{2} \mathrm{~S}_{4}$ by density functional theory (DFT). The authors observed a higher density of states (DOS) near the Fermi level in $\mathrm{Fe}-\mathrm{NiCo}_{2} \mathrm{~S}_{4}$ (Fig. $7 \mathrm{~g}-\mathrm{l}$ ) and hence higher electronic conductivity, mainly ascribed to reduced localization of electrons. This DOS was partially delocalized charged which could be attributed to the lengthened Fe-S bonds (Fig. 7g-1). DFT further revealed that $\mathrm{Fe}-\mathrm{NiCo}_{2} \mathrm{~S}_{4}$ surface held a very higher affinity, or much higher IL electrolyte adsorption enthalpy, and enhanced IL polarization effect, therefore leading to improved electrochemical performance.

\section{APPLICATIONS OF BMCS IN EES SYSTEMS}

The major target of engineered nanomaterials with well-defined morphology, composition, and crystal structure is to optimize or modulate the electronic and physiochemical properties, thus obtaining better electrochemical performance for EES devices. The resultant nanomaterials obtained from morphological, hybrid, and heterostructure engineering have potential application in various EES devices that are very important to overcome the serious issues of climate change and limited resources of fossil fuels (coal, oil, gas, etc.). Keeping in mind these issues, in the following section we will summarize and discuss the potential of the abovementioned engineered materials in SCs, MIBs, and beyond MIBs, with emphasis on those electrode materials having unique charge mechanisms or excellent energy storage performance.

\section{SCs}

SCs have attracted increased attention as a potential candidate 

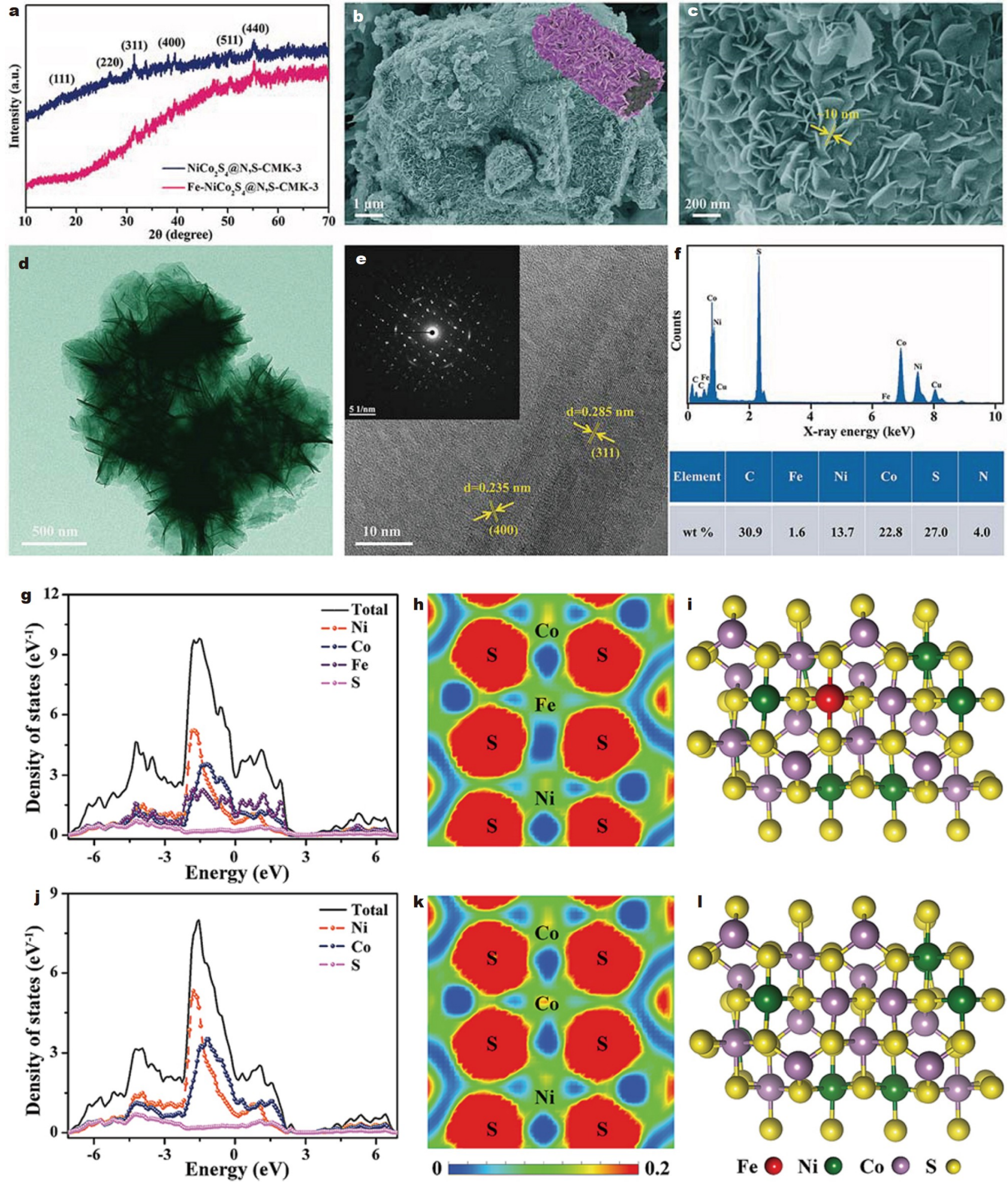

Figure 7 (a-f) Formation of Fe- $\mathrm{NiCo}_{2} \mathrm{~S}_{4}$ : (a) X-ray diffraction (XRD) patterns of $\mathrm{NiCo}_{2} \mathrm{~S}_{4} @ \mathrm{~N}, \mathrm{~S}-\mathrm{CMK}$ and $\mathrm{Fe}-\mathrm{NiCo} \mathrm{S}_{4} @-\mathrm{N}, \mathrm{S}-\mathrm{CMK}$. (b, c) SEM images, (d) TEM image, (e) HRTEM image of Fe- $\mathrm{NiCo}_{2} \mathrm{~S}_{4} @ \mathrm{~N}, \mathrm{~S}-\mathrm{CMK}$, respectively. Inset: SAED pattern. (f) EDS spectrum of Fe- NiCo $\mathrm{S}_{4} @ \mathrm{~N}, \mathrm{~S}-\mathrm{CMK}$ and content of different elements. (g-l) Effect of Fe doping: calculated DOS for (g) $\mathrm{Fe}_{-} \mathrm{NiCo}_{2} \mathrm{~S}_{4}$ and (j) $\mathrm{NiCo}_{2} \mathrm{~S}_{4}$. Electron localization function for (h) Fe-NiCo $\mathrm{S}_{4}$ and (k) $\mathrm{NiCo}_{2} \mathrm{~S}_{4}$. Crystal structure of (i) $\mathrm{Fe}-\mathrm{NiCo}_{2} \mathrm{~S}_{4}$ and (l) $\mathrm{NiCo}_{2} \mathrm{~S}_{4}$. Reprinted with permission from Ref. [217]. Copyright 2019, Royal Society of Chemistry.

for energy storage systems owing to their high power density, fast charging/discharging characteristics, excellent lifespan, and low maintenance cost. They are mainly categorized into two types based on their charge storage mechanisms: electrical double-layer capacitors (EDLC) and pseudocapacitors. EDLCs store charge based on adsorption/desorption at electrode-electrolyte surfaces/interfaces, and usually show high cyclic stability, while Faradaic redox reactions are involved in pseudocapacitors 
and generally deliver high capacitance. The amount of charge storage in SCs strongly depends on their active materials. Among various studied materials, BMC-based active materials have demonstrated high capacitive properties due to their high electrical conductivity, rich active sites, and large mechanical/ thermal stability. However, it is a big challenge to obtain satisfactory energy density and maintain high capacitance at high scan rates or high current density. The rate performance of electrode materials can be improved by simultaneously enhancing the rates of electron transfer and ion diffusion. In this regard, nanostructuring has been approved to develop BMCs to boost capacitive performance. For instance, a nickel manganese sulfide (Ni-Mn-S) hexagonal sheet-in-cage structure with nanosized open spaces was developed to improve redox reactions and decrease charge transfer resistance [49]. The Ni-Mn-S electrode demonstrated a high specific capacitance $\left(1664 \mathrm{~F} \mathrm{~g}^{-1}\right.$ at $1 \mathrm{~A} \mathrm{~g}^{-1}$ and $785 \mathrm{~F} \mathrm{~g}^{-1}$ at $50 \mathrm{~A} \mathrm{~g}^{-1}$ ) due to the synergistic effect between $\mathrm{Mn}$ and Ni electrochemical active sites, and improved electronic/ionic conductivity. Besides, hexagonal sheets might inhibit the breakage of nanocages upon redox reactions, which in turn achieved a high utilization of electrochemical active components.

Hollow nanostructured BMCs featured with high surface area, well-defined walls, well-defined hollow interior, uniform shell thickness, large number of active sites, and outstanding electrolyte/ion permeability can be a fascinating approach to enhance the capacitive performance. Up to now, a variety of BMCs with different compositions (such as Ni-Co-S/Se, Cu-Co$\mathrm{S} / \mathrm{Se}$, Ni-Mn-S/Se, Zn-Mn-S, Co-Mo-S/Se) and diverse hollow structured morphologies (sphere, nanotube, capsule, onion, nanobox, and nanosheet) have been reported for SCs. Compared with other compositions, Ni-Co-S/Se with various crystal structures including $\mathrm{NiCo}_{2} \mathrm{~S}_{4}, \mathrm{NCSe}, \mathrm{CoNi}_{2} \mathrm{~S}_{4}, \mathrm{Ni}_{1.77} \mathrm{Co}_{1.23} \mathrm{~S}_{4}$, $\mathrm{Ni}_{1.5} \mathrm{Co}_{1.5} \mathrm{~S}_{4}$ and $\left(\mathrm{Ni}_{0.33} \mathrm{Co}_{0.67}\right) \mathrm{Se}_{2}$ have been extensively reported and demonstrated to exhibit superior capacitive performance. For instance, Zhang et al. [218] synthesized core-shell $\mathrm{Ni}_{x} \mathrm{Co}_{3-x} \mathrm{~S}_{4}(x=1,1.5$, and 2$)$ micro/nano-spheres and observed the decreasing trend in specific capacity with increasing content of cobalt. The $\mathrm{NiCo}_{2} \mathrm{~S}_{4}$ showed the highest capacity (155 mA h g ${ }^{-1}$ ), while the $\mathrm{Ni}_{1.5} \mathrm{Co}_{1.5} \mathrm{~S}_{4}$ presented superior cyclic stability (74\% capacity retention after 2000 cycles) and excellent rate performance. This concluded that controlling the content of metal elements in BMCs could provide a viable route to achieve desired electrochemical performance. In addition to sulfides, monodisperse hollow NCSe (H-NCSe) microspheres with metallic nature were also prepared as high performance electrode materials for SCs [81]. It demonstrated competitive pseudocapacitance ( $750 \mathrm{~F} \mathrm{~g}^{-1}$ at $3 \mathrm{~A} \mathrm{~g}^{-1}$ after 100 cycles) at ultrahigh mass loading of $7 \mathrm{mg} \mathrm{cm}^{-2}$. The as-assembled activated carbon $(\mathrm{AC}) / \mathrm{H}-\mathrm{NCSe}$-based hybrid device showed high electrochemical performance. A maximum energy density of $25.5 \mathrm{~W} \mathrm{~h} \mathrm{~kg}^{-1}$ at a power density of $3.75 \mathrm{~kW} \mathrm{~kg}^{-1}$ was achieved along with high cycling life of $82.3 \%$ capacity retention after 5200 cyclic voltammetry (CV) charge/discharge cycles (Fig. 8a-e). More interestingly, with a systematic electrochemical and physicochemical investigation, intrinsic EES mechanisms of the $\mathrm{H}-\mathrm{NCSe}$ electrode in aqueous $\mathrm{KOH}$ electrolyte over $\mathrm{CV}$ scanning discovered the electrooxidation-generated biactive $\mathrm{CoOOH} / \mathrm{NiOOH}$ phases, instead of the H-NCSe, which is responsible for the significant pseudocapacitance (Fig. $8 \mathrm{f}-\mathrm{k}$ ).

Integrating active materials with carbonaceous materials are another efficient approach to improve the capacitive performance of BMCs. These carbonaceous materials can not only serve as templates to support the growth of nanostructured BMCs but also improve the electrical conductivity and as well relieve the strain generated during repeated cycling. In the last decade, extensive effort has been made to synthesize the nanocomposites of various kinds of BMCs (Ni-Co-S/Se, Cu-Co-S/Se, $\mathrm{Zn}-\mathrm{Co}-\mathrm{S}, \mathrm{Ni}-\mathrm{Fe}-\mathrm{S}$ ) with traditional carbon materials (such as pyrolytic carbon and natural graphite) and novel carbon materials (such as graphene, CNTs, and CC) to improve the performance of SCs. Among various BMCs, Ni-Co-S/Se is the most common active materials that have been composited with carbonaceous materials. For instance, integrated hybrid architecture consisting of edge site-enriched $\mathrm{Ni}$-Co-S nanoparticles and graphene frameworks ( $\mathrm{Ni}-\mathrm{Co}-\mathrm{S} / \mathrm{G})$ by the in-situ chemically converted process was reported [126]. The Ni-Co-S/G hybrid exhibited high capacitance (1492 $\mathrm{F} \mathrm{g}^{-1}$ at $1 \mathrm{~A} \mathrm{~g}^{-1}$ ), superior rate performance $(96 \%$ retention when the current density was increased from 1 to $50 \mathrm{~A} \mathrm{~g}^{-1}$ ), and outstanding electrochemical stabilities due to intimate contact between $\mathrm{Ni}$-Co-S particles and highly conductive graphene matrices, presence of abundant electroactive active sites in $\mathrm{Ni}-\mathrm{Co}-\mathrm{S}$ edge sites, and strong affinity for $\mathrm{OH}^{-}$in the electrolyte. Moreover, Ni-Co-S/G hybridbased asymmetric SCs exhibited a high energy density of $43.3 \mathrm{~W} \mathrm{~h} \mathrm{~kg}^{-1}$ at a high power density of $22.1 \mathrm{~kW} \mathrm{Kg}^{-1}$. In another report, the influence of CNT contents added to the composite $\left(\mathrm{CNT} / \mathrm{NiCO}_{2} \mathrm{~S}_{4}\right)$ on the capacitive performance was also investigated to get the optimal value [219]. The capacitance of the composites increased with increasing content of CNTs from 0 to $40 \mathrm{mg}$ but decreased from 40 to $60 \mathrm{mg}$. Therefore the addition of $40 \mathrm{mg}$ of CNTs to the composite electrode exhibited the highest capacitance that can be attributed to the synergistic effect of the double-layer capacitance of the CNTs and pseudocapacitance of $\mathrm{NiCo}_{2} \mathrm{~S}_{4}$. However, when the amount of the CNTs exceeded $40 \mathrm{mg}$, the pseudocapacitance of NCS has a smaller effect on the electrodes compared with the double-layer capacitance of the CNTs; thus the electrochemical performance was decreased instead. Apart from Ni-Co-S/Se, the decoration of $\mathrm{Cu}_{2} \mathrm{CoS}_{4}$ nanoparticles on nitrogen-doped graphene $\left(\mathrm{Cu}_{2} \mathrm{CoS}_{4} /\right.$ $\mathrm{NG}$ ) was also presented in which NG presents a high specific surface area to decrease the self-accumulation and confines the shape of $\mathrm{Cu}_{2} \mathrm{CoS}_{4}$ nanoparticles to boost the electrochemical performance [130].

The afore-mentioned BMCs/carbonaceous composites usually require dead elements (conductive agent, binders, and current collectors) to prepare the electrodes. These additional elements not only increase the weight of the device but also block the active sites and result in poor rate performance. To avoid the dead elements and completely utilize the electrochemically active materials, nanostructured BMCs with various morphologies such as nanoparticles [141,172,220], burl-like [168], nanowires [221], nanorods [175], nanotubes [222,223], nanoneedles $[170,224]$, nanowedges [176], and nanosheets [158,161,162,166] have been directly deposited on diverse 3D bulk and highly conductive templates as integrated electrodes for SCs. One of the first related studies was reported by Chen et al. [222], in which a self-standing $\mathrm{NiCo}_{2} \mathrm{~S}_{4}$ nanotube array was in-situ deposited on $\mathrm{Ni}-\mathrm{F}$ by hydrothermal steps and used as a free-standing electrode for SCs. The $\mathrm{NiCo}_{2} \mathrm{~S}_{4}$ nanotube array on the Ni-F effectively lowers the inactive components and enhances the surface area of active materials due to the ultrathin wall which can get 

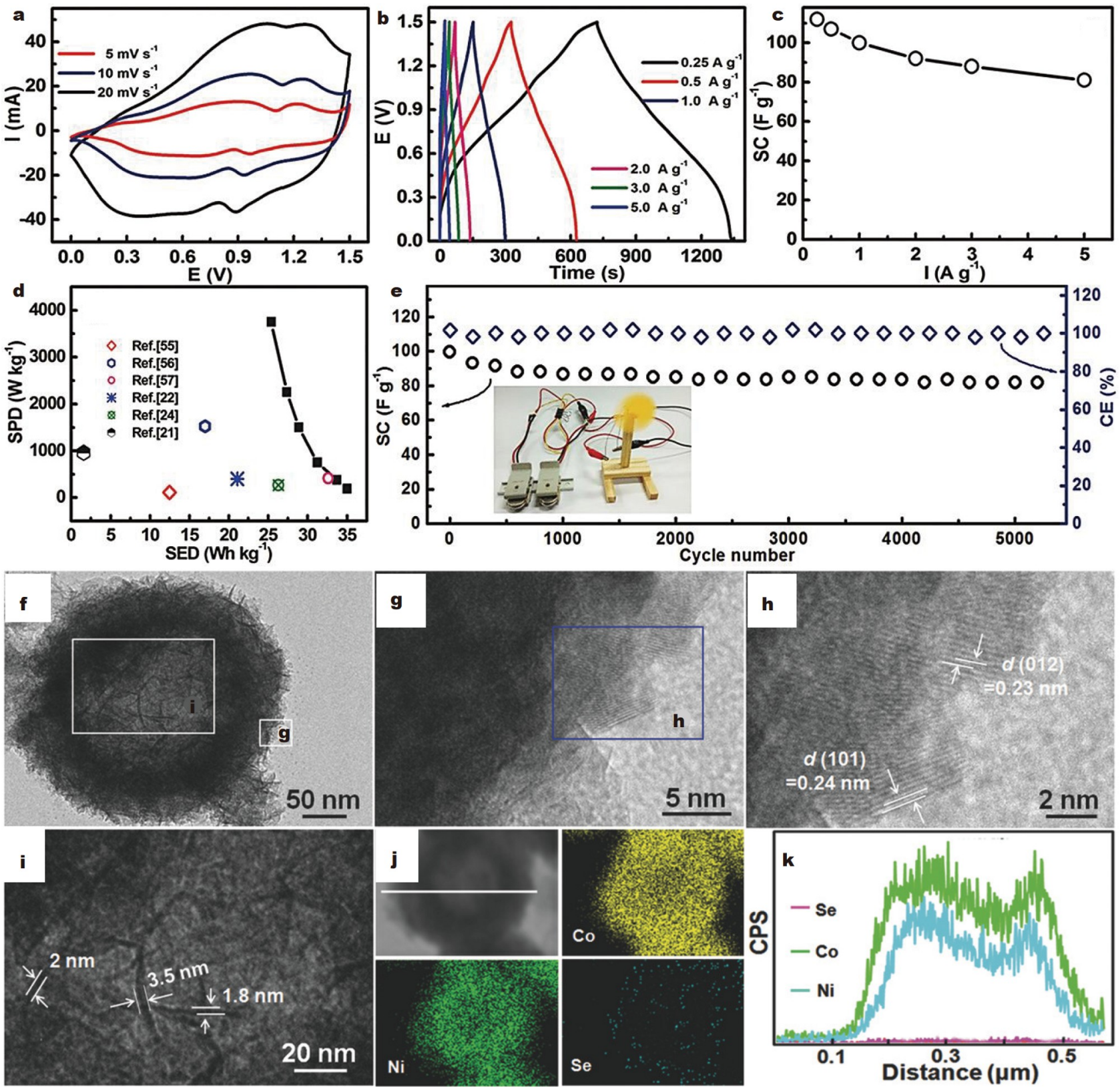

Figure 8 (a-e) Electrochemical properties of the assembled AC//H-NCS hybrid device: (a) CV curves. (b) Chronopotentiometry (CP) profiles. (c) Specific capacitance versus current density. (d) Ragone plot compared with other selenide-based asymmetric cells. (e) Cycling behaviors along with the Coulombic efficiency profile. The inset in panel (e) shows an electric fan powered by the hybrid cells in parallel. (f-k) Morphological and structural evaluation after 100 CV cycles: (f) TEM image. (g, h) HRTEM images, (i) Magnified TEM image of rectangle region in panel (a). (j) EDS elemental mapping images acted on an individual NCSe sub-microspheres after CV cycles. (k) Linear sweep EDS signals along the white line in panel (j) as indicated. The images in panels (g-i) are the magnified images of rectangle regions in panels $(\mathrm{f})$ and $(\mathrm{g})$ as indicated. Reprinted with permission from Ref. [81]. Copyright 2018, John Wiley and Sons.

high utilization of redox sites at high active mass loading. At a high electroactive material mass loading $\left(6 \mathrm{mg} \mathrm{cm}^{-2}\right)$, the hybrid electrode presented an ultrahigh capacitance $\left(14.39 \mathrm{~F} \mathrm{~cm}^{-2}\right.$ at $5 \mathrm{~mA} \mathrm{~cm}^{-2}$ ) with excellent rate capability (67.7\% capacitance retention at $150 \mathrm{~mA} \mathrm{~cm}^{-2}$ ) and cycling performance $(92 \%$ retention after 5000 cycles). Excitingly, the $\mathrm{NiCo}_{2} \mathrm{~S}_{4}$-based asymmetric SCs delivered a high areal capacity $\left(4.68 \mathrm{~F} \mathrm{~cm}^{-2}\right.$ at $\left.10 \mathrm{~mA} \mathrm{~cm}{ }^{-2}\right)$, energy density $\left(31.5 \mathrm{~W} \mathrm{~h} \mathrm{~kg}^{-1}\right.$ at $\left.156.6 \mathrm{~W} \mathrm{~kg}^{-1}\right)$, and power density $\left(2348.5 \mathrm{~W} \mathrm{~kg}^{-1}\right.$ at $\left.16.6 \mathrm{~W} \mathrm{~h} \mathrm{~kg}^{-1}\right)$. Recently, $\mathrm{NiCo}_{2} \mathrm{~S}_{4}$ nanoparticles with average sizes of $20-25 \mathrm{~nm}$ were uniformly anchored on a $3 \mathrm{D}$ compressible CNT sponge by a simple hydrothermal process and used as the compressible electrode for SCs [220]. The mass loading of $\mathrm{NiCo}_{2} \mathrm{~S}_{4}$ was changed by simply altering the hydrothermal reaction time and the highly dense $\mathrm{NiCo}_{2} \mathrm{~S}_{4}$ nanoparticles with a smoother surface on the CNT sponge were obtained with an optimal reaction time of $10 \mathrm{~h}$ and presented superior electrochemical performance (Fig. 9a-g). Most impressively, the assembled asymmetric SC with $\mathrm{NiCo}_{2} \mathrm{~S}_{4} / \mathrm{CNT}$ sponge as cathode and $\mathrm{Fe}_{2} \mathrm{O}_{3} / \mathrm{CNT}$ sponge as anode, not only exhibited a high specific capacitance of $117 \mathrm{~F} \mathrm{~g}^{-1}$ at $1 \mathrm{~A} \mathrm{~g}^{-1}$, the superior energy density of $41.6 \mathrm{~W} \mathrm{~h} \mathrm{~kg}^{-1}$, and excellent cyclic stability ( $82 \%$ after 5000 cycles upon $80 \%$ strain), but also displayed good compression and flexibility (Fig. 9h-m). Contrary to simple nanostructured BMCs on $3 \mathrm{D}$ bulk materials, 

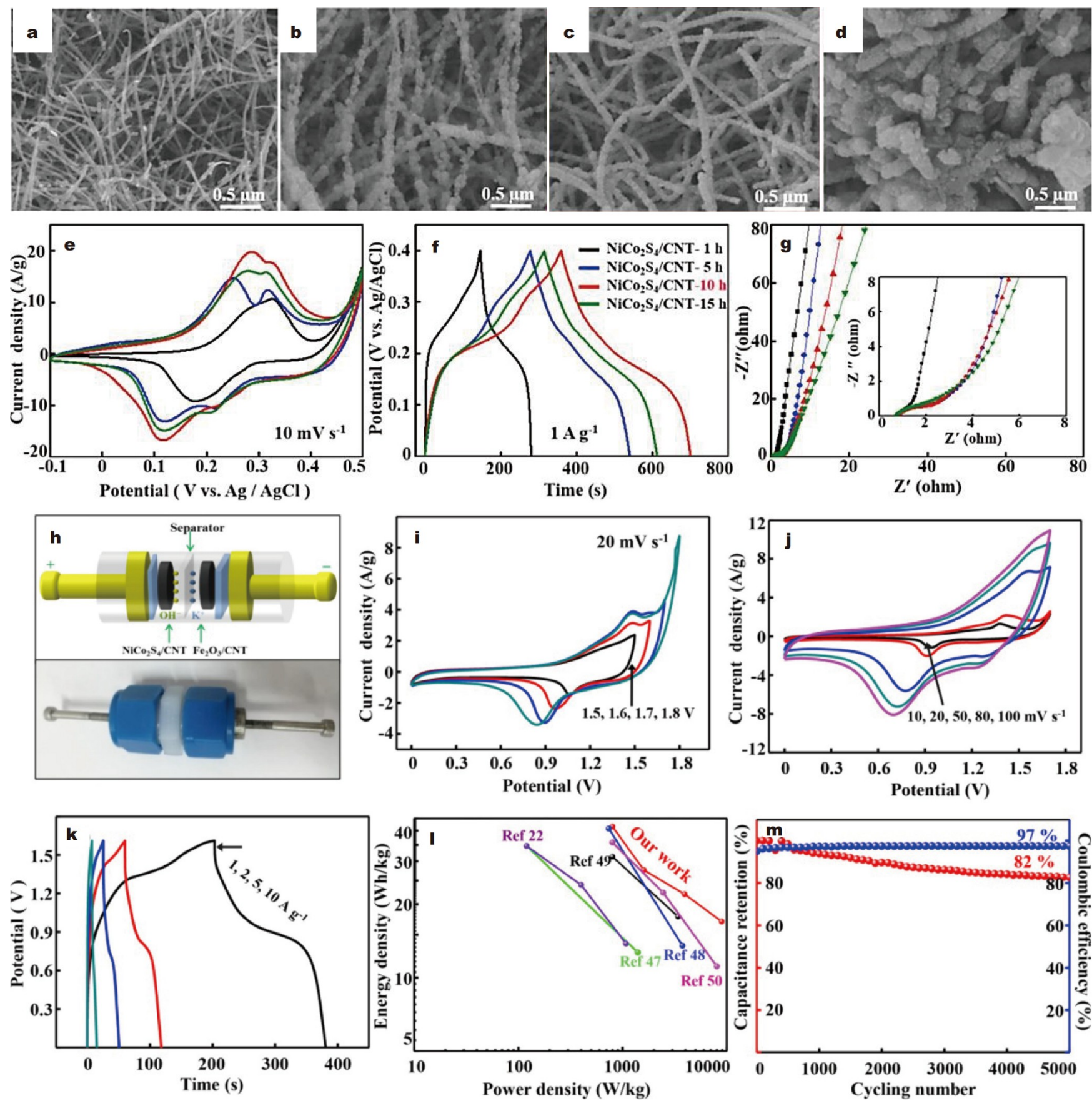

Figure 9 (a-g) The influence of mass loading of $\mathrm{NiCO}_{2} \mathrm{~S}_{4}$ on the electrochemical properties: (a-d) SEM images and (e-g) the corresponding electrochemical properties in $3 \mathrm{~mol} \mathrm{dm}^{-3} \mathrm{KOH}$ of $\mathrm{NiCo}_{2} \mathrm{~S}_{4} / \mathrm{CNT}$ sponge electrodes by solvothermal treating at $180^{\circ} \mathrm{C}$ for diverse reaction times: (a) $1 \mathrm{~h}$, (b) $5 \mathrm{~h}$, (c) $10 \mathrm{~h}$, (d) $15 \mathrm{~h}$, (e) CV curves, (f) galvanostatic discharge/charge (GCD) curves, and (g) Nyquist plots of electrochemical impedance spectroscopy. (h-m) The capacitive performance of $\mathrm{NiCo}_{2} \mathrm{~S}_{4} / \mathrm{CNT} / / \mathrm{Fe}_{2} \mathrm{O}_{3} / \mathrm{CNT}$ device: (h) schematic diagram and optical image of a highly compressible asymmetric SC, (i) CV curves in different potential windows, (j) CV curves at various scan rates, (k) GCD curves at different current densities between 0 and $1.6 \mathrm{~V}$, (l) the Ragone plots, and (m) the cycle properties of the device. Reprinted with permission from Ref. [220]. Copyright 2021, American Chemical Society.

hybrid BMCs with hierarchical nanoarchitectures have also been grown on $3 \mathrm{D}$ conductive templates, signifying improved capacitive performances $[102,179,225-229]$. Such kind of structures not only further increased the conductivity, but also was expected to enhance the electroactive sites. For instance, $\mathrm{Zn}_{0.76} \mathrm{Co}_{0.24} \mathrm{~S}$ nanoparticles embedded in $\mathrm{NC}$ were directly deposited on flexible CC and used as the electrode for SC with high pseudocapacitive charge storage [102]. The optimized $\mathrm{Zn}_{0.76} \mathrm{Co}_{0.24} \mathrm{~S}$-NC electrode delivered a high capacitance of
2132.2 $\mathrm{F} \mathrm{g}^{-1}$ at $1.25 \mathrm{~A} \mathrm{~g}^{-1}$, excellent rate performance, and exceptional high cyclic stability $(95.63 \%$ capacitance retention after 10,000 cycles).

Furthermore, hierarchical compound composites consisting of BMCs and high pseudocapacitive materials have displayed better electrochemical properties compared with a single component. These compound composite materials provide multiple reactive equivalents, intriguing hetero-interfaces, distinctive ion-permeable bulk microstructure, fast pathways for ion/electrolyte 
transfer, and appealing synergetic structural/compositional/ componental contributions, resulting in enhanced electrochemical performance. Until now, many electroactive materials including metal oxides ( $\mathrm{NiO}$ [196], $\mathrm{MnO}_{2}$ [230], $\mathrm{NiMoO}_{4}$ [184], $\mathrm{NiCoO}_{2}$ [193], $\mathrm{Ni}_{3} \mathrm{~V}_{2} \mathrm{O}_{8}$ [231], $\mathrm{CoMoO}_{4}$ [232]), metal hydroxides $\left(\mathrm{Co}(\mathrm{OH})_{2} \quad[192,199,233], \mathrm{Ni}(\mathrm{OH})_{2} \quad[188,201,202,234], \mathrm{Ni}-\mathrm{Mn}\right.$ LDH [235,236], Ni/Co LDHs [237], FeOOH [230], $\mathrm{Co}_{x} \mathrm{Ni}_{1-x^{-}}$ $(\mathrm{OH})_{2}$ [230], $\mathrm{NiCo}_{2}\left(\mathrm{CO}_{3}\right)_{1.5}(\mathrm{OH})_{3}$ [238]), metal sulfides $\left(\mathrm{CoS}_{x}\right.$ [239], $\mathrm{CoS}_{2}$ [183], $\mathrm{Co}_{3} \mathrm{~S}_{4}$ [240], $\mathrm{Co}_{9} \mathrm{~S}_{8}$ [181,189,241], $\mathrm{MoS}_{2}$ [242]), metal selenides $\left(\mathrm{MoSe}_{2}\right.$ [27,243], $\mathrm{Ni}_{3} \mathrm{Se}_{2}$ [19]), metal phosphides (NiP [198], NiFeP [191]), and polypyrrole [244,245] have been combined with diverse BMCs as high performance electrodes for SCs. Most of these diversified heterostructured BMCs nanoarrays are grown on highly conductive substrates such as $\mathrm{Ni}-\mathrm{F}$, graphene, $3 \mathrm{D}$ graphene sponge, carbon paper, and CC. As a typical example, $\mathrm{FeCo}_{2} \mathrm{~S}_{4} @ \mathrm{Ni}(\mathrm{OH})_{2}$ with a hierarchical layer-by-layer 3D network was deposited on Ni-F and presented excellent specific capacitance $\left(2984 \mathrm{~F} \mathrm{~g}^{-1}\right.$ at $\left.5 \mathrm{~mA} \mathrm{~cm}^{-2}\right)$ and cycling stability $(95.7 \%$ after 5000 cycles) due to the distinctive layer-by-layer structure and the synergy between $\mathrm{FeCo}_{2} \mathrm{~S}_{4}$ and $\mathrm{Ni}$ $(\mathrm{OH})_{2}$ nanosheets [202]. The all-solid-state asymmetric SC based on $\mathrm{FeCo}_{2} \mathrm{~S}_{4} @ \mathrm{Ni}(\mathrm{OH})_{2} / / \mathrm{rGO}$ delivered a maximum energy density of $64 \mathrm{~W} \mathrm{~h} \mathrm{~kg}-1$, a maximum power density of $10.2 \mathrm{~kW} \mathrm{~kg}^{-1}$ and outstanding cycling performance $(92.9 \%$ after 10,000 cycles). Moreover, compound composites of two dissimilar BMCs have also been developed as electrode to obtain satisfying capacitive performance $[187,194,199,203,204,246,247]$. For example, the nanotube-built multitripod arrays of dissimilar BMCs composite $\left(\mathrm{FeCo}_{2} \mathrm{~S}_{4}-\mathrm{NiCo}_{2} \mathrm{~S}_{4}\right)$ on a silver-sputtered textile cloth was fabricated [246]. The $\mathrm{FeCo}_{2} \mathrm{~S}_{4}-\mathrm{NiCo}_{2} \mathrm{~S}_{4}$ was used as flexible electrode for SCs and showed superior capacitive per- formance than individual components $\mathrm{MCo}_{2} \mathrm{~S}_{4}$, which was ascribed to the synergy of Faradaic redox reaction of individual components $\left(\mathrm{FeCo}_{2} \mathrm{~S}_{4}\right.$ and $\left.\mathrm{NiCo}_{2} \mathrm{~S}_{4}\right)$. The free standing $\mathrm{FeCo}_{2} \mathrm{~S}_{4}{ }^{-}$ $\mathrm{NiCo}_{2} \mathrm{~S}_{4}$ electrode delivered high capacitance $\left(1519 \mathrm{~F} \mathrm{~g}^{-1}\right.$ at $5 \mathrm{~mA} \mathrm{~cm}^{-2}$ ), and excellent rate capability (85.1\% retention at $40 \mathrm{~mA} \mathrm{~cm}^{-2}$ ), attributed to highly porous and robust unique multitripod architectures. Moreover, the assembled flexible allsolid-state symmetric device showed high energy density (46 $\mathrm{W} \mathrm{h} \mathrm{kg}^{-1}$ at $1070 \mathrm{~W} \mathrm{~kg}^{-1}$ ), and high cycling stability with capacitance retention of $92 \%$ upon 3000 cycles (Fig. 10a-f). Interestingly, this flexible device can be twisted and showed excellent reliability/stability with no obvious capacitance degradation under huge twisting configuration, indicating its merit in wearable electronic devices (Fig. 10g, h). Impressively, the area of the device can be adjusted by connecting such flexible capacitors in series and the capacitance increased linearly with increasing area of the device, signifying the possibility of combining of SCs to meet different energy output requirements (Fig. 10i-k).

\section{MIBs}

MIBs such as LIBs, SIBs, and PIBs have been serving humankind for the past several decades as one of the most efficient energy storage systems and assist in the realization of smart and portable technology due to their high energy density, pollution-free operation, higher efficiencies, and environmental benignity. Many state-of-the-art nanostructured electrode materials for MIBs have been developed to enhance their electrochemical storage. For example, mono TMCs like $\mathrm{MoS}_{2}, \mathrm{MoSe}_{2}, \mathrm{SnS}_{x}, \mathrm{FeS}_{x}$, $\mathrm{FeSe}_{x}, \mathrm{CoS}_{x}, \mathrm{CoSe}_{\mathrm{x}}$ have been widely investigated as attractive anode materials for LIBs, SIBs, and PIBs. In recent years, BMCs
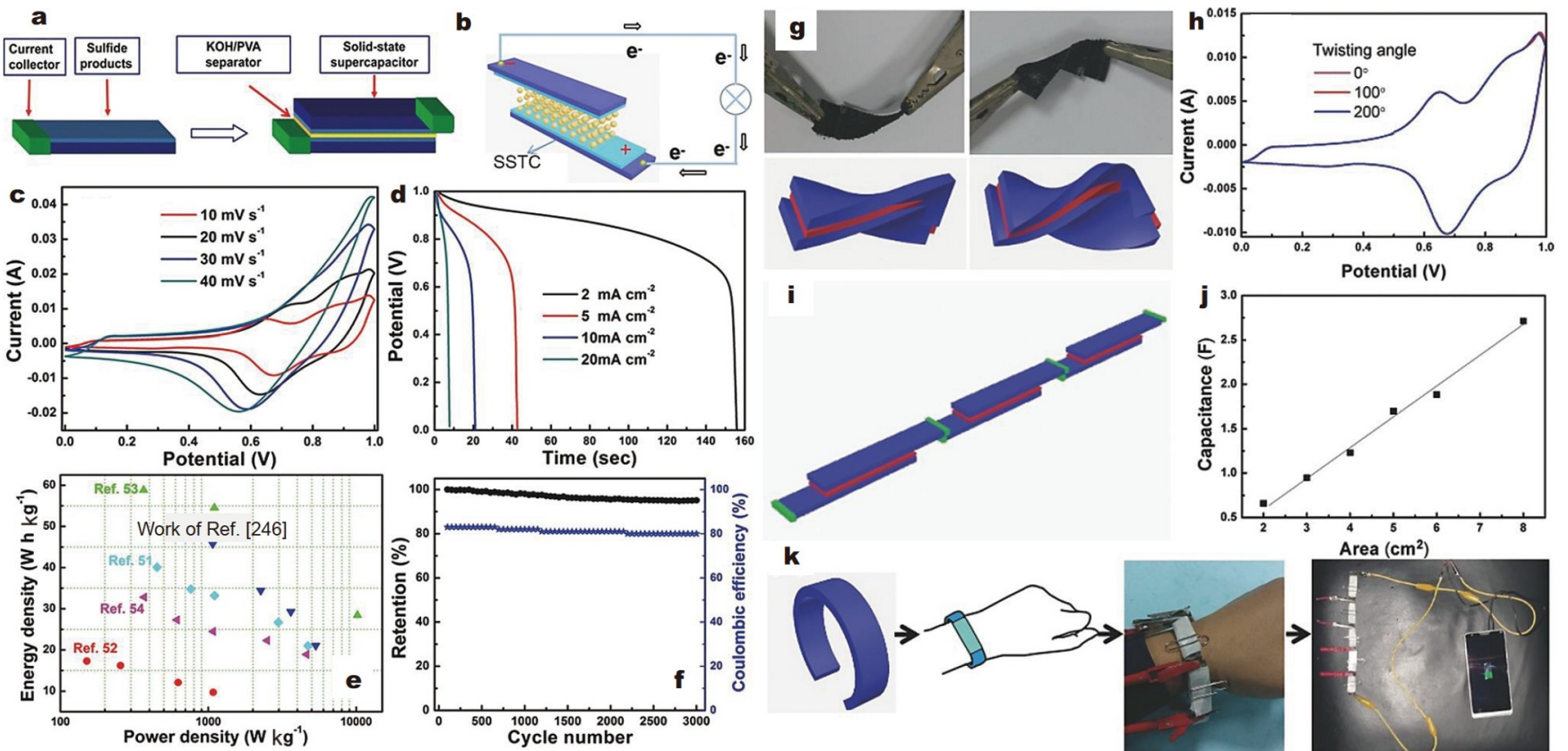

Figure 10 Schematic illustrations of (a) assembled structure of an all-solid-state symmetric SC and (b) electron transfer in a working capacitor. (c) CV curves at various scan rates and (d) charge-dischage curves in the potential range from 0 to $1.0 \mathrm{~V}$ of the symmetric device with the $\mathrm{FeCo}_{2} \mathrm{~S}_{4}-\mathrm{NiCo}_{2} \mathrm{~S}_{4}$ electrode. (e) Ragone plots of the device and some other reported SCs. (f) Capacitance retention and Coulombic efficiency over 3000 charge-discharge cycles at a current density of $10 \mathrm{~mA} \mathrm{~cm}{ }^{-2}$. (g) Digital photos and schematic diagrams of an all-solid-state device under different twisting angles. (h) CV curves of solid-state SC at different twisting angles. (i) Schematic illustration of the connection of SCs in series. (j) Areal capacitance of single $\mathrm{FeCo}_{2} \mathrm{~S}_{4}-\mathrm{NiCo}_{2} \mathrm{~S}_{4}$ electrode as a function of the textile area measured at the discharge current of $1 \mathrm{~mA}$. (k) Schematic illustration of wristband made by connecting five SCs devices in series, and a digital photo showing a mobile phone charged by the SC-integrated wristband. Reprinted with permission from Ref. [246]. Copyright 2017, John Wiley and Sons. 
with higher electrical conductivity and specific capacity have attracted enormous attention and display better alkali $\left(\mathrm{Li}^{+}, \mathrm{Na}^{+}\right.$, $\mathrm{K}^{+}$) ion storage properties than mono TMCs. Nevertheless, the conversion/alloying reaction of BMCs with alkali ions causes a large volume expansion which produces the strain and delaminates the active material from the current collector, leading to rapid capacity fade and decreased cyclic stability. Nanostructure engineering can be an efficient way to tackle this critical problem and effectively improve ionic kinetics. Using the nanostructure engineering strategy, extensive effort has been devoted to producing high performance BMC-based electrode materials for MIBs $[86,91,93,94,182,248-250]$. For instance, by employing $\mathrm{ZnCo}_{2}$-glycolate microspheres as a template, hollow $\mathrm{Zn}_{0.33} \mathrm{Co}_{0.67} \mathrm{~S}$ microspheres were prepared via an ion exchange process and used as anode for both LIBs and SIBs [66]. They exhibited a reversible capacity of $540 \mathrm{~mA} \mathrm{~g}^{-1}$ at $0.5 \mathrm{~A} \mathrm{~g}^{-1}$ after 200 cycles for LIBs, whereas a discharge capacity of $420 \mathrm{~mA} \mathrm{~h} \mathrm{~g}^{-1}$ at current density of $0.5 \mathrm{~A} \mathrm{~g}^{-1}$ over 50 sodiation/desodiation cycles was achieved [66]. The high $\mathrm{Li} / \mathrm{Na}$ storage performance was ascribed to the hollow interior that not only serves as a reservoir for the electrolyte to deliver high $\mathrm{Li} / \mathrm{Na}$ ion flux across the shortest distance, but also accommodates the volume variation upon repeated $\mathrm{Li} / \mathrm{Na}$ insertion/extraction. In general, most of the BMCs usually involved the conversion/alloying reaction mechanism along charge storage at a lower voltage, resulting in fast capacity fading due to huge volume variation. Recently, Yan et al. [86] discovered a stepwise intercalation-conversion-intercalation reaction mechanism in $\mathrm{CuInS}_{2}$ using in/ex-situ spectroscopy and microscopy techniques (Fig. 11a-h). The reversible $\mathrm{Na}$ intercalation-extraction reaction within the low voltage range was aroused due to the introduction of indium species in $\mathrm{CuInS}_{2}$ and resulted in high electrochemical stability. Specifically, in the initial sodiation process, first the $\mathrm{Na}^{+}$ion was intercalated to the $\mathrm{CuInS}_{2}$, and subsequently conversion reaction exhibited the generation of $\mathrm{Cu}, \mathrm{Na}_{2} \mathrm{~S}$ and $\mathrm{Na}_{y}-\mathrm{In}_{6} \mathrm{~S}_{7}$ with an enormous contribution of capacity. Lastly, within a low voltage range, the generated $\mathrm{Na}_{y}-\mathrm{In}_{6} \mathrm{~S}_{7}$ contributed to a stable $\mathrm{Na}^{+}$intercalationextraction process. Due to this unique reaction mechanism, $\mathrm{CuInS}_{2}$ showed superior cycling stability compared with $\mathrm{Cu}_{2} \mathrm{~S}$ (for $\mathrm{CuInS}_{2}$, the capacity decay per cycle within 1000 cycles at current density of $5 \mathrm{~A} \mathrm{~g}^{-1}$ was $0.027 \%$, while this was $0.325 \%$ loss per cycle at $2 \mathrm{~A} \mathrm{~g}^{-1}$ in case of $\mathrm{CuS}_{2}$ ) (Fig. 11i).

The schematic of overall reaction mechanism is descried as follows:

Sodiation process:

intercalation:

$\mathrm{CuInS}_{2}+x \mathrm{Na}^{+}+x \mathrm{e}^{-} \rightarrow \mathrm{Na}_{x} \mathrm{CuInS}_{2}$,

conversion:

$6 \mathrm{Na}_{x} \mathrm{CuInS}_{2}+(10+y-6 x) \mathrm{Na}^{+}+(10+y-6 x) \mathrm{e}^{-}$

$\rightarrow 5 \mathrm{Na}_{2} \mathrm{~S}+\mathrm{Na}_{y} \operatorname{In}_{6} \mathrm{~S}_{7}+6 \mathrm{Cu}$,

intercalation:

$\mathrm{Na}_{y} \mathrm{In}_{6} \mathrm{~S}_{7}+z \mathrm{Na}^{+}+z \mathrm{e}^{-} \rightarrow \mathrm{Na}_{y+z} \operatorname{In}_{6} \mathrm{~S}_{7}$

Desodiation process:

deintercalation:

$\mathrm{Na}_{y+z} \operatorname{In}_{6} \mathrm{~S}_{7} \rightarrow \mathrm{Na}_{a} \operatorname{In}_{6} \mathrm{~S}_{7}+(y+z-a) \mathrm{Na}^{+}+(y+z-a) \mathrm{e}^{-}$,

conversion and desodiation:
$6 \mathrm{Cu}+\mathrm{Na} \operatorname{In}_{6} \mathrm{~S}_{7}+5 \mathrm{Na}_{2} \mathrm{~S} \rightarrow$

$6 \mathrm{Na}_{b} \mathrm{CuInS}_{2}+(10+a-6 b) \mathrm{Na}^{+}+(10+a-6 b) \mathrm{e}^{-}$.

This unique charge storage mechanism efficiently improves the kinetics to obtain excellent reversibility and rate performance. Taking into account the novel functions and diversity of BMCs, this unique stepwise reaction process can also be applied in other BMCs to achieve high battery performance.

The fabrication of nanohybrids with different carbonaceous materials is another effective strategy to alleviate the volume variation of BMC-based electrode materials for alkali ion storage due to the strong mechanical flexibility of these carbon materials. The carbon materials can also enhance the electronic conductivity of resultant nanohybrids, resulting in high rate capability, and improved cyclic stability, and prohibit the selfaggregation of BMC nanostructures. The carbonaceous materials can be integrated with BMCs in two ways: (i) combining of BMCs with pyrolytic carbon, (ii) growth of BMCs with various carbonaceous matrices. Generally, the pyrolytic carbon is in amorphous form and has been extensively used to in/ex-situ decorate the BMCs for alkali ion storage [104,116]. For example, hollow mesoporous carbon nanospheres embedded with ultrafine cobalt iron selenides were reported as an anode material for PIBs. The as-made hybrid structure showed a high specific capacity $\left(485 \mathrm{~mA} \mathrm{~h} \mathrm{~g}^{-1}\right.$ after 200 cycles at $100 \mathrm{~mA} \mathrm{~g}^{-1}$ ) and excellent rate performance $\left(272 \mathrm{~mA} \mathrm{~h} \mathrm{~g}^{-1}\right.$ at $\left.2000 \mathrm{~mA} \mathrm{~g}^{-1}\right)$ [115]. The ultrafine bimetallic selenides confined within abundant pores not only mitigate the volume expansion but also prohibit the aggregation of nanoparticles, whereas hollow mesoporous carbon nanospheres act as conductive templates and structural skeletons. Furthermore, a hierarchical porous $\mathrm{Fe}_{2} \mathrm{CoSe}_{4}$ structure impeded into a thin carbon matrix was prepared via an exclusive approach and presented high and stable capacity in Na-ion half and full-cells [108]. This material showed excellent cycling stability ( $350 \mathrm{~mA} \mathrm{~h} \mathrm{~g}^{-1}$ at $4000 \mathrm{~mA} \mathrm{~g}^{-1}$ after 5000 cycles) in a Naion half-cell and a specific capacity of $247.6 \mathrm{~mA} \mathrm{~h} \mathrm{~g}^{-1}$ at $200 \mathrm{~mA} \mathrm{~g}^{-1}$ up to 100 cycles in a Na-ion full-cell (Fig. 12a-e).

The growth of BMCs with different carbonaceous matrices including graphene, CNTs and CNFs can also be an intriguing strategy to improve the $\mathrm{Li} / \mathrm{Na} / \mathrm{K}$ ion storage performance. Among various carbonaceous matrices, graphene is intensively hybridized with BMCs for use in LIBs/SIBs/PIBs. For example, a $\mathrm{SnCoS}_{4} /$ graphene hybrid was fabricated via a hydrothermal method and used as an anode for LIB [129]. This $\mathrm{SnCoS}_{4}$ /graphene hybrid exhibited a high reversible capacity of $940 \mathrm{~mA} \mathrm{~h} \mathrm{~g}^{-1}$ at a high current density of $2000 \mathrm{~mA} \mathrm{~g}^{-1}$ after 2000 cycles, which is higher than that of $\mathrm{SnS}_{2}$ /graphene and $\mathrm{CoS}_{2}$ /graphene [129]. The superior lithium storage of $\mathrm{SnCoS}_{4} /$ graphene was ascribed to smaller sizes of $\mathrm{SnCoS}_{4}$ hybrid nanocrystals and well dispersion onto graphene nanosheets, which provide more interfaces and active sites. Moreover, the production of Co nanoparticles during the electrochemical conversion process of $\mathrm{SnCoS}_{4}$ can enhance electrode kinetics and cyclic stability. Till now, many other reports have shown the fabrication of $\mathrm{CuCo}_{2} \mathrm{~S}_{4}-\mathrm{rGO}[127,131], \mathrm{MnSb}_{2} \mathrm{~S}_{4}-\mathrm{rGO}$ [119], $\mathrm{Ni}_{3} \mathrm{Co}_{6} \mathrm{~S}_{8}$-rGO [124], $\mathrm{Cu}_{2} \mathrm{SnS}_{3}$-rGO [128], $\mathrm{Cu}_{2} \mathrm{Sn}_{3} \mathrm{~S}_{7} / \mathrm{Cu}_{2} \mathrm{SnS}_{3}-$ rGO [252], and yolk-shell heterostructured $\mathrm{Ni}_{2} \mathrm{P}_{1-x} \mathrm{~S}_{x} / \mathrm{Ni} @$ carbon/grapheme [190] composites for LIBs/SIBs/PIBs with improved reversible capacities and/or cycling/rate performances. Interestingly, single/or dual heteroatom doping into rGO can tune the electrical and electrochemical properties of rGO, which 


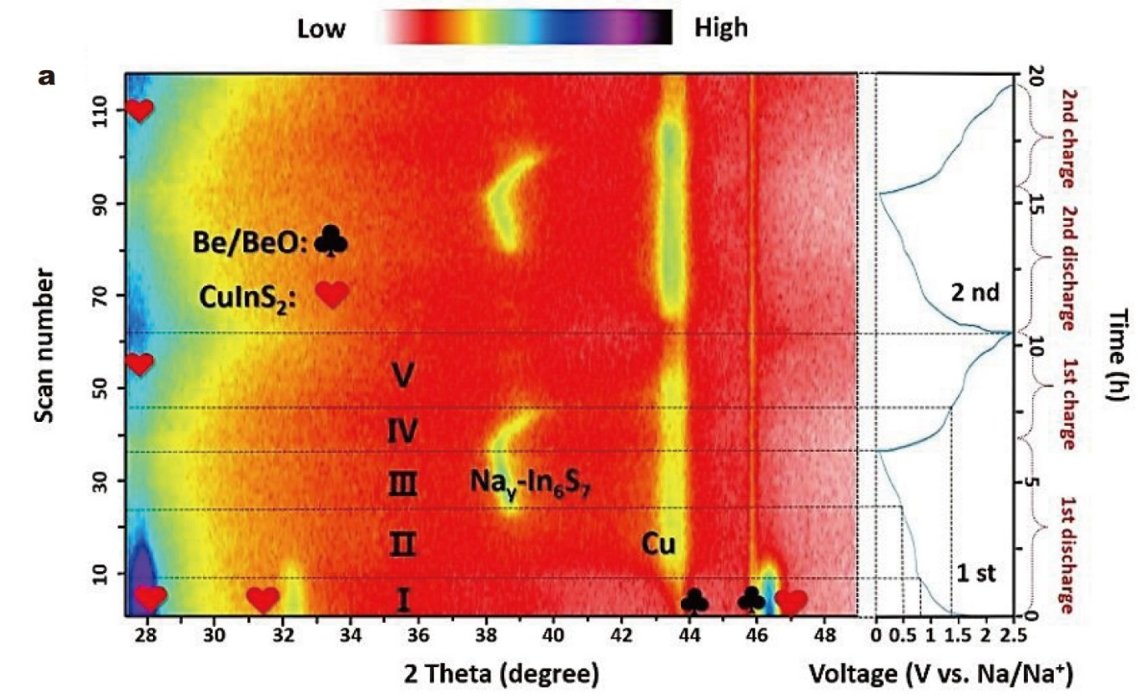

b

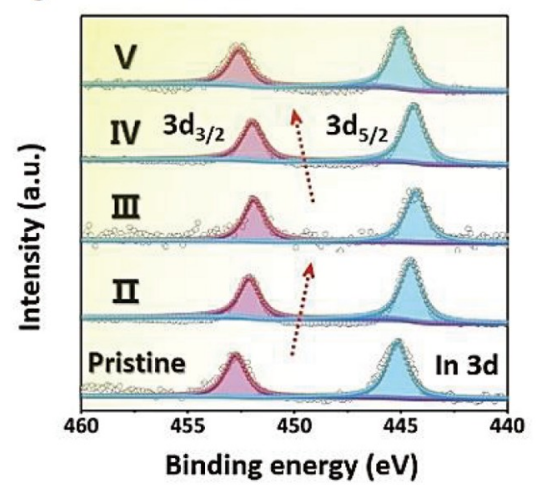

f

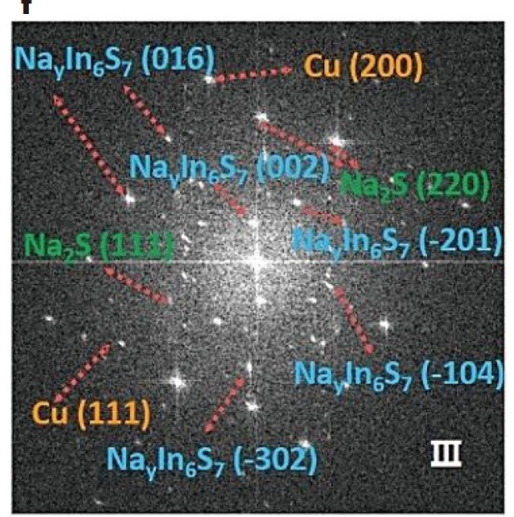

d

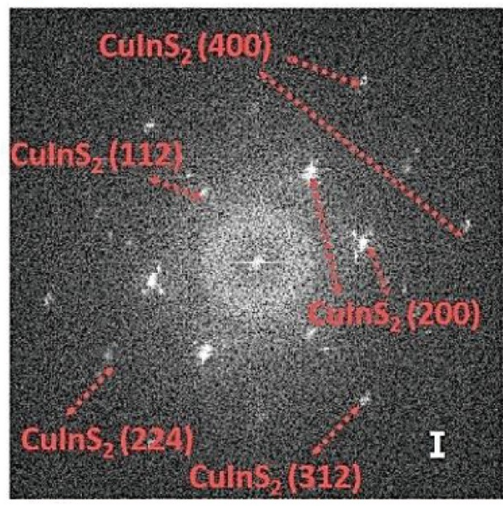

g

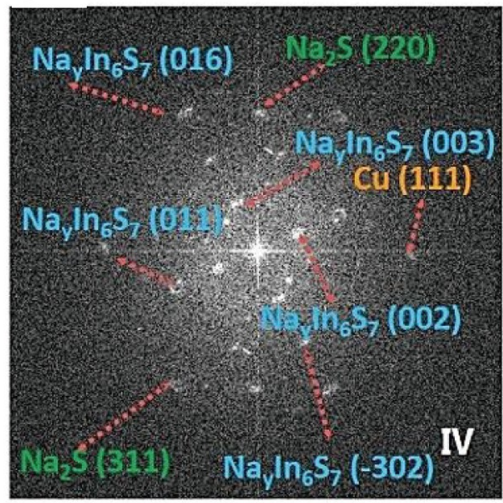

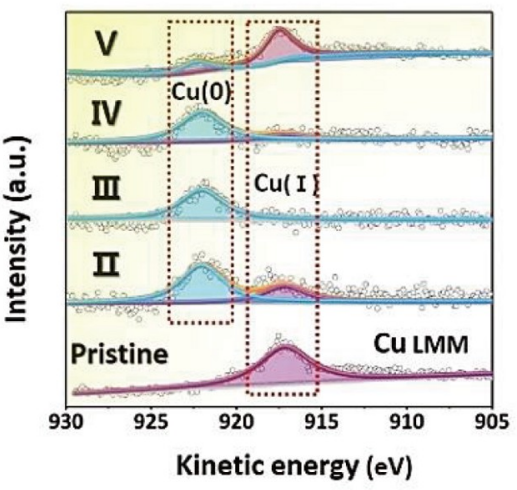

e

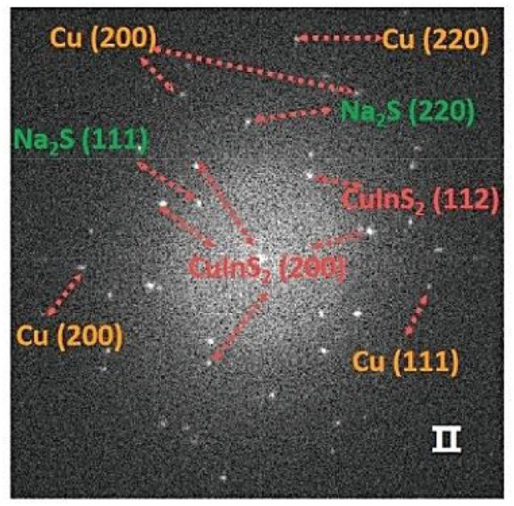

h

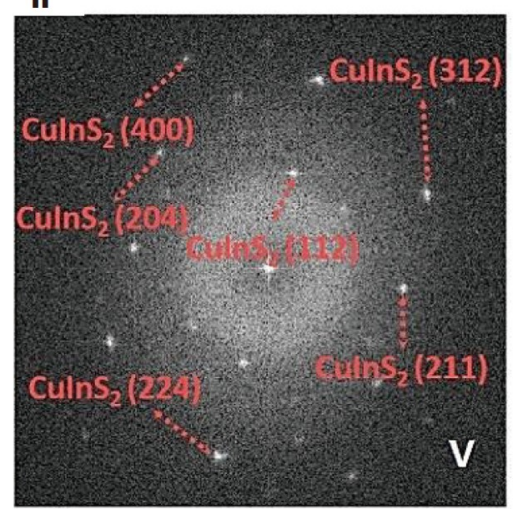

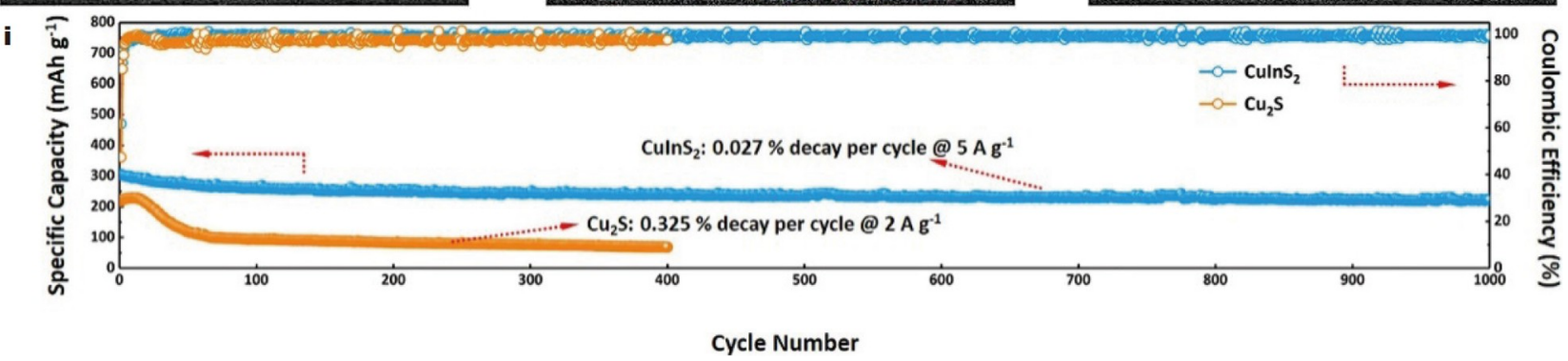

Figure 11 (a-h) Reaction mechanism of CuInS 2 as SIB anode: (a) in situ XRD 2D contour plot. XPS spectra of (b) Cu LMM, and (c) In 3d at different reaction stages. (d-h) Fast Fourier transform patterns derived from ex-situ HRTEM at different reaction stages. (i) The comparison of cycling stabilities of $\mathrm{CuInS}_{2}$ and $\mathrm{Cu}_{2} \mathrm{~S}$. Reprinted with permission from Ref. [86]. Copyright 2020, American Chemical Society. 
can further assist in improving the $\mathrm{Li} / \mathrm{Na}$ storage [132]. For instance, Liu et al. [122] fabricated the N/S dual-doped rGO encapsulating hollow $\mathrm{ZnSnS}_{3}$ cube to improve the slow kinetics, unsatisfactory rate performance and poor cyclic stability of metal sulfides. As an anode for SIB, this design demonstrated a high discharge capacity (501 $\mathrm{mA} \mathrm{h} \mathrm{g}^{-1}$ at $100 \mathrm{~mA} \mathrm{~g}^{-1}$ after 100 cycles) and an excellent reversible capacity $\left(290.7 \mathrm{~mA} \mathrm{~h} \mathrm{~g}^{-1}\right.$ at $1000 \mathrm{~mA} \mathrm{~g}^{-1}$ after 500 cycles) [122]. In addition to 2D graphene, hybrid nanostructures of BMCs with other carbon matrices such as $1 \mathrm{D}$ CNTs and CNFs, have also been used as anodes for LIBs/ SIBs, and demonstrated increased charge storage performances than their individual counterparts due to good conductivity of CNTs/CNFs and the high electrochemical activity of BMCs $[136,137,142,253]$. Besides carbonaceous conductive matrices, a recent study is reported in which conductive polypyrrole (PPy) was decorated on $\mathrm{CuCo}_{2} \mathrm{~S}_{4}$ with a core-shell like structure and applied as an anode electrode for half and full-cell LIBs with enhanced performance [254].

The charge storage performance of BMCs for LIBs/SIBs can also be improved by growing the nanostructured arrays on $3 \mathrm{D}$ conductive templates including CC [164,251], 3D graphene foam [148], 3D graphene aerogel [255], carbon foam [160], CFP [171], Ni-F [256-259] to make free standing electrodes and eliminate the dead elements (binder and conductive agents, etc.). These $3 \mathrm{D}$ templates provide a high surface area, excellent conductivity, and flexibility to hybrid structures. Besides, the $3 \mathrm{D}$ network of nanostructured arrays provides a high specific surface area, enough void space, shortened pathways for charge transport, easy diffusion of electrolyte, and strong mechanical resilience. As a typical example, a 3D network array of $\mathrm{NiCo}_{2} \mathrm{~S}_{4}$ nanosheets was grown on CC via a hydrothermal route followed by a sulfurization process and used as free standing anode for LIB [251]. This $\mathrm{NiCO}_{2} \mathrm{~S}_{4} / \mathrm{CC}$ array delivered an excellent lithium storage performance including outstanding discharge capacity $\left(1275 \mathrm{~mA} \mathrm{~h} \mathrm{~g}^{-1}\right.$ at $\left.1000 \mathrm{~mA} \mathrm{~g}^{-1}\right)$, good cyclic stability $\left(1137 \mathrm{~mA} \mathrm{~h} \mathrm{~g}^{-1}\right.$ after 100 cycles at $\left.1000 \mathrm{~mA} \mathrm{~g}^{-1}\right)$, and high rate capability $\left(340 \mathrm{~mA} \mathrm{~h} \mathrm{~g}^{-1}\right.$ at $\left.5000 \mathrm{~mA} \mathrm{~g}^{-1}\right)$. Excitingly, in-situ TEM was used to investigate the mechanical strain produced in $\mathrm{NiCo}_{2} \mathrm{~S}_{4}$ during the lithiation process. During the lithiation process, the $\mathrm{NiCo}_{2} \mathrm{~S}_{4}$ nanosheet continued to expand and after full lithiation, a total volume expansion of $60 \%$ was observed (after $20 \mathrm{~min}$ ) (Fig. 12f-i). The relatively small volume expansion, along with negligible changes in dimension and morphology, no cracking or fracture of $\mathrm{NiCo}_{2} \mathrm{~S}_{4}$ nanosheets after full lithiation, was attributed to the mesoporous nature of nanosheets [251].

However, all the BMCs' structures prepared in the abovementioned composites are non-layered. The alkali ion diffusion rate can be further accelerated by designing the layered structure with large interlayer spacings, highlighting the significance of expanded interlayer spacing enabling faster ionic kinetics. So far only a few studies have reported on alkali ion storage of fewlayered BMCs with a wide interlayer spacing [60,85]. For example, Chen et al. [60] prepared hollow nanospheres consisting of a layered CMS material and amorphous carbon (CMS1) with large interlayer distance $(\sim 0.51 \mathrm{~nm}$ for $(002)$ crystal plane), promoting the rate capability and cyclic stability. Interestingly, using ex situ XPS and in situ X-ray absorption spectroscopy, they discovered the conversion mechanism in the carbonate-based electrolyte, while intercalation mechanism in ether-electrolyte, thereby allowing fast $\mathrm{Na}^{+}$storage perfor- mances. Moreover, with the introduction of rGO in CMS1 composite, the resultant CMS1-rGO composite still preserved the hollow structural integrity with smaller charge transfer resistance $\left(R_{\mathrm{ct}}\right)$, thus improving the rate performance $\left(131.9 \mathrm{~mA} \mathrm{~h}^{-1}\right.$ at $\left.5000 \mathrm{~mA} \mathrm{~g}^{-1}\right)$ and cyclic stability (95.6\% after 2000 cycles), while with the full cell, still excellent cycling stability (75.5\% after 500 cycles) was achieved.

Besides monovalent cation batteries (LIBs/SIBs/PIBs), rechargeable ion batteries based on multivalent cations such as $\mathrm{Mg}^{2+}, \mathrm{Al}^{3+}, \mathrm{Zn}^{2+}$ can be the future energy storage devices due to their high energy density, low cost, and relatively large abundance in the Earth's crust [260-263]. The working principle of multi-IBs is similar to that of mono-valent ion batteries (LIBs/ SIBs/PIBs) in many ways, except the use of multivalent ion instead of mono-valent ion. Recently, BMCs have also been explored as cathode materials for multi-IBs [74,79,118]. For instance, CMS is a layered type material that has been used as conversion-type anode for LIBs/SIBs currently; it is demonstrated that layered structure of I-phase CMS with different morphologies like nanotubes, nanosheets and hollow polyhedra can serve as an ideal host for reversible $\mathrm{Mg}^{2+}$ intercalation/deintercalation [79]. It was discovered from ex-situ XRD, Raman, XPS, and TEM results that the crystal plane (002) expands/ contracts during $\mathrm{Mg}^{2+}$ intercalation/de-intercalation processes $3.12 \AA$ (Mg-intercalated) and $3.06 \AA$ (Mg-deintercalaed) accompanied by the valence state change of $\mathrm{Mo}$ and $\mathrm{Cu}$ elements (Fig. 13a-h). Moreover, it was further demonstrated that hollow polyhedral CMS (CMS-H) nanocages showed better $\mathrm{Mg}^{2+}$ ion storage compared with CMS nanosheets (CMS-S), attributed to ultra-thin walls of the CMS-H, which can accommodate the strain and facilitate short-range charge transport pathways, signifying the privilege of hollow structure beyond nanosheets (Fig. 13i, j) [79].

Aluminum ion battery (AIBs) is another kind of multi-IBs; however, the development of highly efficient cathode materials for AIBs is at the preliminary stage. Recently it was reported that the LDHs derived BMCs can be an effective strategy to develop high performance cathodes for AIBs [118]. For example, Xing et al. [118] synthesized a composite consisting of nickel-cobalt sulfides derived from LDH and rGO (S-NiCo@rGO) and investigated it as a cathode material for AIBs using the ionic electrolyte. Owing to a well-designed hybrid structure, the SNiCo@rGO composite exhibited high discharge capacity $\left(248 \mathrm{~mA} \mathrm{~h} \mathrm{~g}^{-1}\right.$ at $\left.1000 \mathrm{~A} \mathrm{~g}^{-1}\right)$ and capacity retention (83 $\mathrm{mA} \mathrm{h} \mathrm{g}^{-1}$ after 100 cycles). The ex-situ experiment further revealed that during the discharge/charge process $\mathrm{Al}^{3+}$ ions might be intercalated/de-intercalated instead of large-sized $\mathrm{AlCl}_{4}{ }^{-}$cations and the charge storage mechanism of S-NiCo may attribute to the substitution of $\mathrm{Ni}^{2+}$ and $\mathrm{Co}^{2+} / \mathrm{Co}^{3+}$ with $\mathrm{Al}^{3+}$ [118]. Based on the above discussion, the charge/discharge reaction mechanism of S-NiCo@rGO could be described as follows:

During the discharge process:

$4 \mathrm{Al}_{2} \mathrm{Cl}_{7}{ }^{-} \rightarrow 7 \mathrm{AlCl}_{4}{ }^{-}+\mathrm{Al}^{3+}$,

$\mathrm{NiS}+m \mathrm{Al}^{3+}+3 m \mathrm{e}^{-} \rightarrow \mathrm{Al}_{m} \mathrm{Ni}_{n} \mathrm{~S}+(1-n) \mathrm{Ni}$,

$\mathrm{Co}_{3} \mathrm{~S}_{4}+p \mathrm{Al}^{3+}+3 p \mathrm{e}^{-} \rightarrow \mathrm{Al}_{p} \mathrm{Co}_{q} \mathrm{~S}_{4}+(3-q) \mathrm{Co}$.

During the charge process:

$7 \mathrm{AlCl}_{4}{ }^{-}+\mathrm{Al}^{3+} \rightarrow 4 \mathrm{Al}_{2} \mathrm{Cl}_{7}{ }^{-}$, 

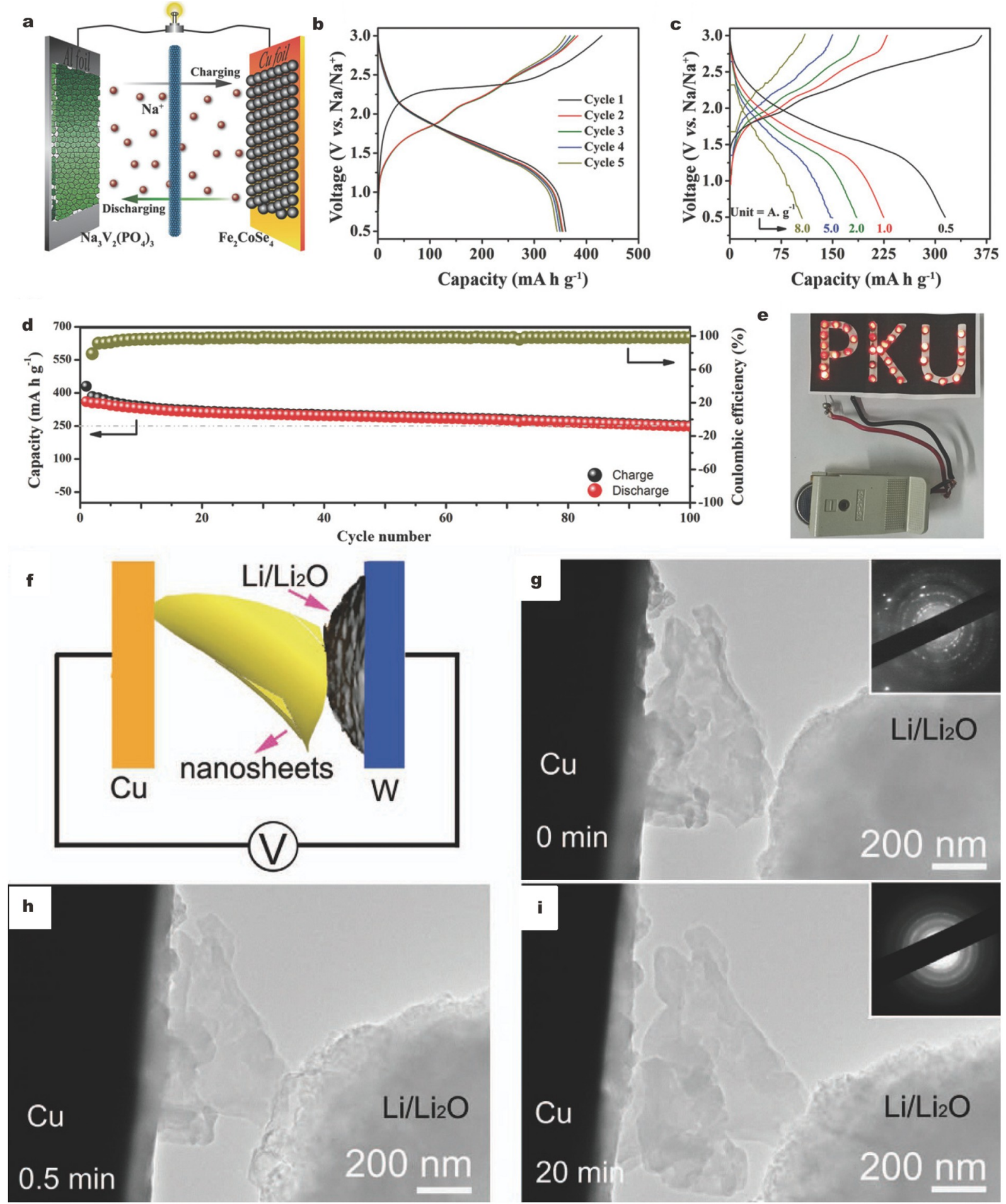

Figure 12 (a-e) Electrochemical evaluation of $\mathrm{Na}_{3} \mathrm{~V}_{2}\left(\mathrm{PO}_{4}\right)_{3}(\mathrm{NVP}) / \mathrm{Fe}_{2} \mathrm{CoSe}_{4}$-based full cell: (a) schematic illustration of full cell. (b) Charge/discharge profiles at $0.2 \mathrm{~A} \mathrm{~g}^{-1}$. (c) Charge/discharge profiles at different current rates. (d) Cycling performance at $0.2 \mathrm{~A} \mathrm{~g}^{-1}$. (e) An array of commercial red lightemitting diodes powered by the NVP/ $\mathrm{Fe}_{2} \mathrm{CoSe}_{4}$ full cell. Reprinted with permission from [108]. Copyright 2018, John Wiley and Son. (f-i) In-situ TEM observation of the lithiation process in $\mathrm{NiCo}_{2} \mathrm{~S}_{4}$ anode: (f) schematic illustration of in-situ TEM cell. (g-i) TEM images monitoring the lithiation process of $\mathrm{NiCo}_{2} \mathrm{~S}_{4}$ nanosheet at time equal to $0,0.5$ and 20 min, respectively. The insets in (g, i) show the corresponding SAED. Reprinted with permission from Ref. [251]. Copyright 2015, Nature Publishing Group.

$\mathrm{Al}_{m} \mathrm{Ni}_{n} \mathrm{~S}+(1-n) \mathrm{Ni}-3 m \mathrm{e}^{-} \rightarrow \mathrm{NiS}+m \mathrm{Al}^{3+}$,

$\mathrm{Al}_{p} \mathrm{Co}_{q} \mathrm{~S}_{4}+(3-q) \mathrm{Co}-3 p \mathrm{e}^{-} \rightarrow \mathrm{Co}_{3} \mathrm{~S}_{4}+p \mathrm{Al}^{3+}$.

\section{MABs}

Although a lot of progress has been made in MIBs, current MIBs are still suffering from inadequate energy density or longevity requirement. Therefore, novel electrochemical batteries such as 

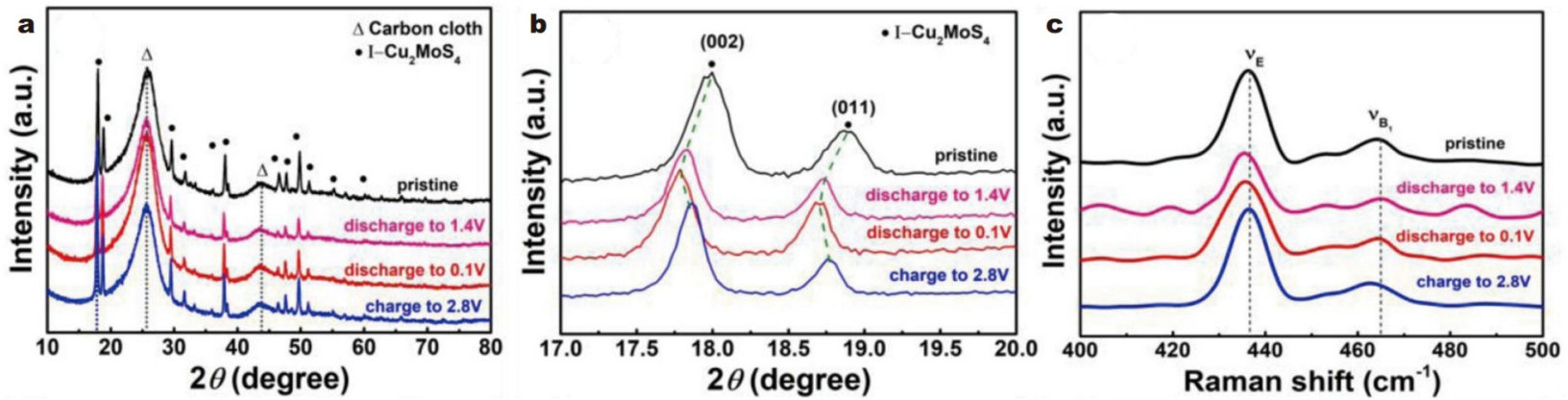

d
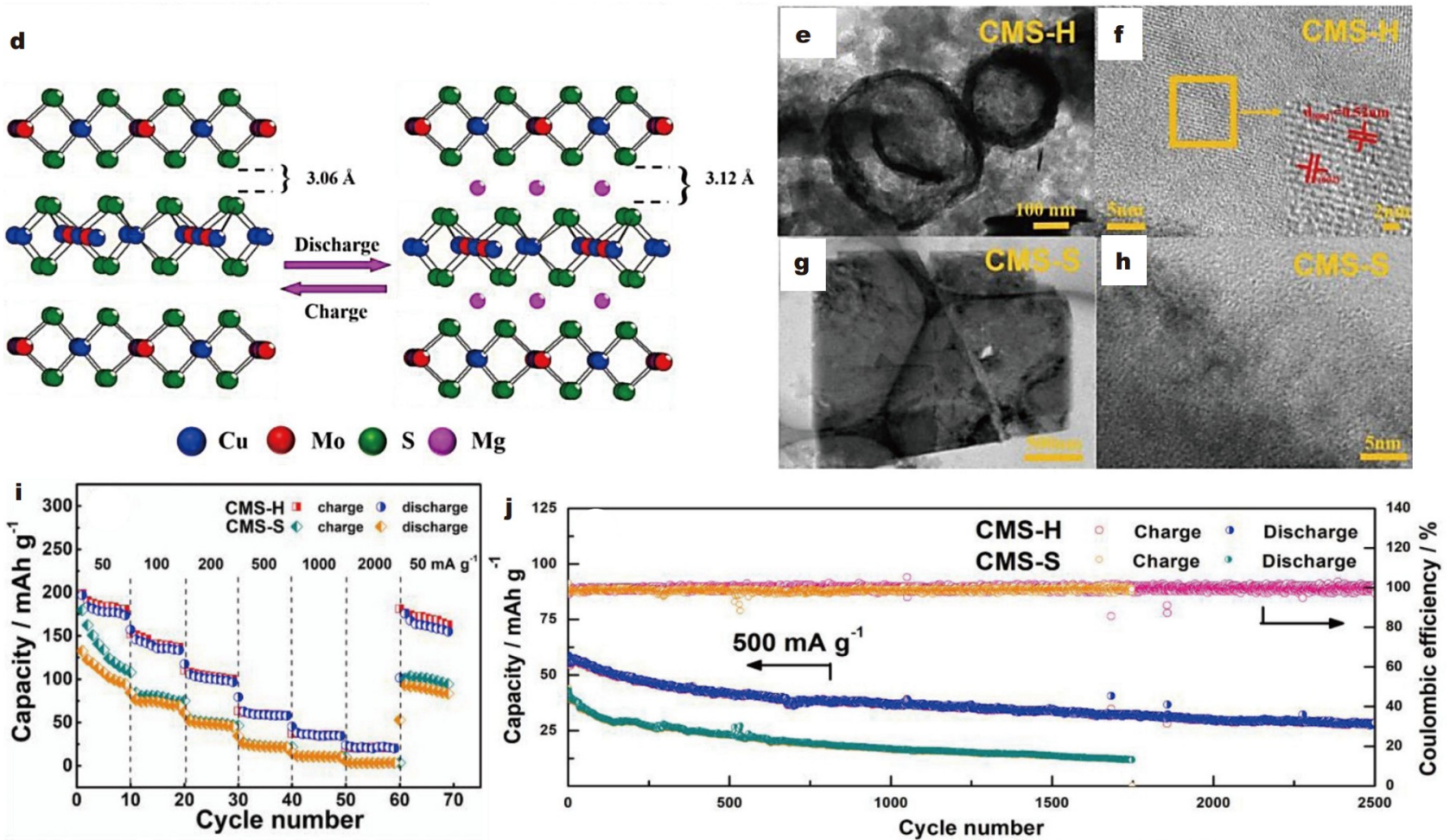

Figure 13 Mg-storage mechanism of CMS: ( $(a, b)$ ex-situ XRD patterns and (c) Raman patterns of CMS-H electrodes after 10 cycles. (d) Schemes illustrating the magnesiation/demagnesiation processes. (e) TEM and (f) corresponding HRTEM images of cycled CMS-H at discharge state (0.1 V) after 10 cycles. (g) TEM and (h) corresponding HRTEM images of CMS-S at discharge state (0.1 V) after 10 cycles. (i) Rate performance of CMS-H and CMS-S. (j) Long-term cycling performance of CMS-H and CMS-S at $500 \mathrm{~mA} \mathrm{~g}^{-1}$. Reprinted with permission from Ref. [79]. Copyright 2020, Elsevier B.V.

MABs including lithium-air batteries (Li-air) and Zn-air batteries (ZAB) are highly required due to their much higher energy density $\left(3500 \mathrm{~W} \mathrm{~h} \mathrm{~kg}^{-1}\right.$ for Li-air battery, $1218 \mathrm{~W} \mathrm{~h} \mathrm{~kg}^{-1}$ for ZAB). Nevertheless, the development of MABs is at the preliminary stage and neither of them is commercialized due to many scientific challenges. For instance, the sluggish reaction kinetics (ORR and OER) at air cathode with high overpotential leads to wastage of energy and poor cyclic stability. To compensate this issue and to reduce the overpotential, many catalysts such as noble metals, metal sulfides/selenides have been reported. Among these, BMC-based nanostructures have been demonstrated to be one of the most promising catalysts for MABs. The high electronic conductivity and presence of more active sites in BMCs are the two main factors to improve catalytic efficiency $[114,167,185,195,264,265]$. However, very few studies have been reported for the application of BMCs as air cathode for Li-air batteries. Primarily, Wang et al. [265] synthesized a graphene-based composite as a bifunctional air electrocatalyst with multiple active species including $\mathrm{Co}_{8} \mathrm{FeS}_{8}$,
$\mathrm{CoFe}_{2} \mathrm{O}_{4}$, and $\mathrm{Co}$, Fe and $\mathrm{N}$ co-doped graphene, presenting outstanding OER and ORR electrocatalytic activities for Li-air batteries. Recently, a highly efficient free-standing air cathode was made consisting of mesoporous $\mathrm{CoNi}_{2} \mathrm{~S}_{4}$ nanorod arrays directly growing on the CT (CNS-RAs/CT) for Li-air battery [167]. The large surface area and open space in the reported CNS-RAs/CT provided abundant void volume to the deposition and accommodation of copious $\mathrm{Li}_{2} \mathrm{O}_{2}$ discharge products, and numerous catalytic sites for OER and ORR.

ZABs are another kind of emerging MABs, and have attracted much attention due to lower cost and abundant reserves of $\mathrm{Zn}$ metal than that of Li metal. Compared with Li-air batteries, extensive investigations for the use of BMCs as air cathode for ZAB have been reported. For example, Qian et al. [80] used the nanostructured engineering strategy and fabricated hollow $\mathrm{Ni}_{x} \mathrm{Co}_{1-x} \mathrm{Se}$ nanocages as the air-cathode for ZAB. The $\mathrm{Ni}_{0.2} \mathrm{Co}_{0.8} \mathrm{Se}$ nanocages showed a stable open-circuit potential $(1.44 \mathrm{~V})$ that is approximately near to that of the precious metal $\mathrm{Pt} / \mathrm{C}+\mathrm{RuO}_{2}$ electrode $(1.46 \mathrm{~V})$, signifying its high catalytic 
performance. Among the $\mathrm{Ni}_{x} \mathrm{Co}_{1-} \mathrm{Se}_{\mathrm{Se}}$ series, $\mathrm{Ni}_{0.2} \mathrm{Co}_{0.8} \mathrm{Se}$ exhibited excellent charge-discharge performance, ultralong cycling lifetime $(>50 \mathrm{~h})$, higher power density $\left(223.5 \mathrm{~mW} \mathrm{~cm}^{-2}\right)$, and a high round-trip efficiency $(60.86 \%)$, indicating its superiority for practical ZAB application (Fig. 14a-c). Besides the outstanding performance of $\mathrm{Ni}_{0.2} \mathrm{Co}_{0.8} \mathrm{Se}$ in the liquid cell, it also demonstrated good performance in industrialized all-solid-state ZAB with a power density of $41.03 \mathrm{~mW} \mathrm{~cm}^{-2}$ (Fig. $14 \mathrm{~d}-\mathrm{i}$ ). The excellent performance of $\mathrm{Ni}_{0.2} \mathrm{Co}_{0.8} \mathrm{Se}$ in $\mathrm{ZABs}$ was largely ascribed to its good electrocatalytic activity. Moreover, a rationally designed hybrid structure with core@shell heterostructure deposited on $\mathrm{MoS}_{2} / \mathrm{N}-\mathrm{S}$ co-doped graphene $\left(\mathrm{MoS}_{2} /\right.$ NSG) can be an efficient strategy to engineer physicochemical properties, thereby improving the electrocatalytic performance. However, such kind of structures of BMCs for MABs is seldom reported. Currently, a heterostructure of small core@shell $\mathrm{Co}_{x} @ \mathrm{CMS}$ decorated on $\mathrm{MoS}_{2} / \mathrm{NSG}$ hetero network was reported and used as the cathodic catalyst for ZABs [185]. It exhibited a high cell voltage $(\sim 1.44 \mathrm{~V})$ and a high power density $\left(40 \mathrm{~mW} \mathrm{~cm}{ }^{-2}\right.$ at $58 \mathrm{~mA} \mathrm{~cm}^{-2}$ ) that was better than a cathodic Pt/
C-based device $\left(32 \mathrm{~mW} \mathrm{~cm}^{-2}\right.$ at $\left.50 \mathrm{~mA} \mathrm{~cm}^{-2}\right)$. This high $\mathrm{ZAB}$ performance was attributed to the synergistic effect of the highly active core@shell $\mathrm{Co}_{x} @ \mathrm{CMS}$ heterostructure with high surface area and high porosity of $\mathrm{MoS}_{2} / \mathrm{NSG}$ hetero network.

Besides $\mathrm{ZAB}$, a hybrid Zn battery that combines the advantage of both $\mathrm{ZAB}$ and alkaline $\mathrm{Zn}$ battery, representing superior environmental adaptability and satisfactory energy and power densities can be the potential candidate for next-generation energy storage systems. The electrochemical performance of a hybrid $\mathrm{Zn}$ battery depends on the multifunctional cathode material that can simultaneously store the charge via Faradaic reaction and as well can perform electrocatalytically. Although BMCs have shown potential application in energy storage devices, BMCs as cathode material for hybrid $\mathrm{Zn}$ batteries are rarely reported. Recently, Cui et al. [195] reported the porous $\mathrm{NCSe} @ \mathrm{NiOOH} / \mathrm{CoOOH}(\mathrm{NCSe} @ \mathrm{NCH})$ heterostructures, obtained from NCSe by an in-situ electrochemical phase transformation route and used as a multifunctional cathode material for the flexible hybrid $\mathrm{Zn}$ battery. Upon phase transformation, the structural integrity of NCSe nanosheets was well preserved
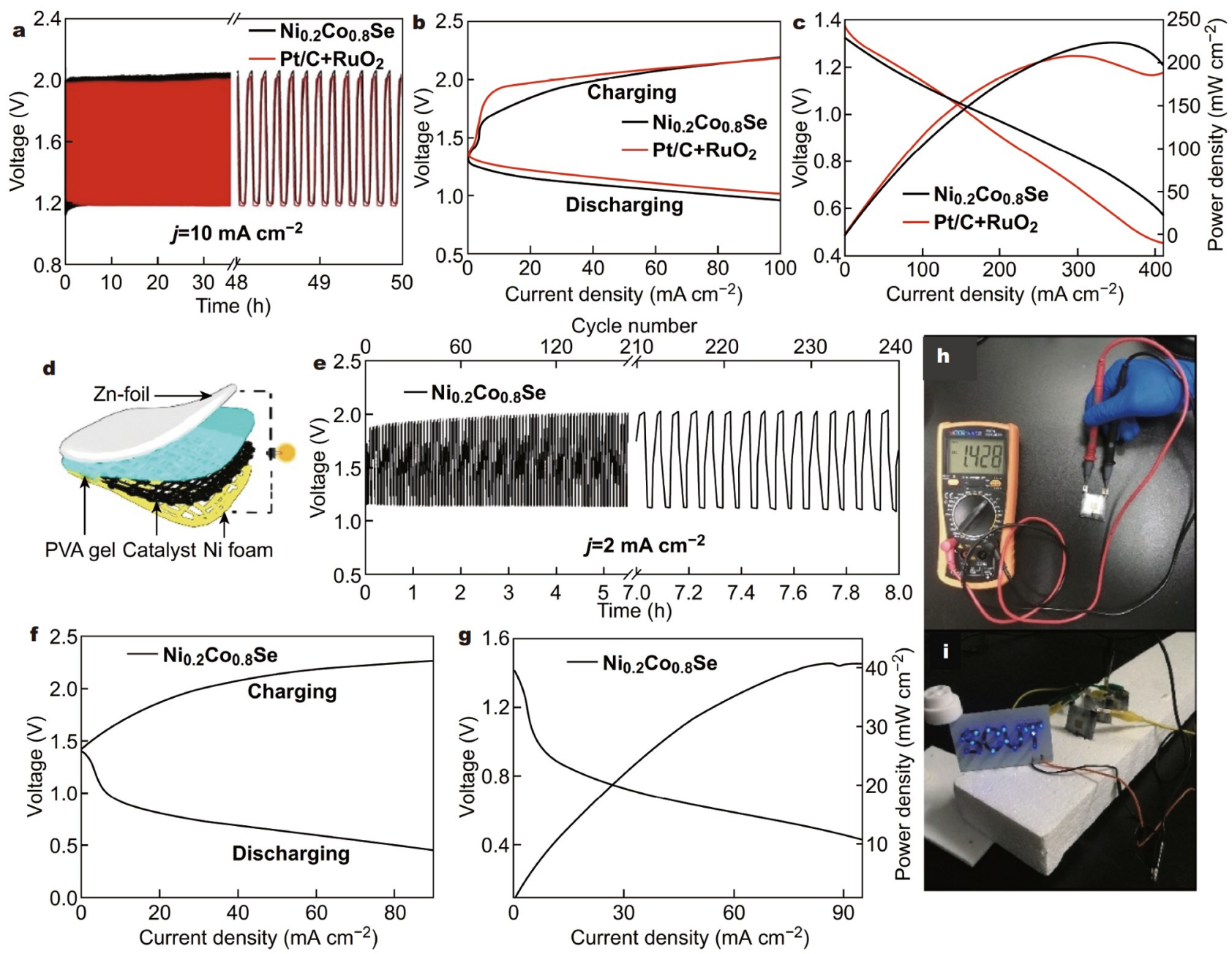

Figure 14 (a) Cycling performance of the rechargeable $\mathrm{ZAB}$ using $\mathrm{Ni}_{0.2} \mathrm{Co}_{0.8} \mathrm{Se}$ at $10 \mathrm{~mA} \mathrm{~cm}^{-2}$, where each cycle lasted $5 \mathrm{~min}$. (b) Charge-discharge polarization curves of the rechargeable $\mathrm{ZABs}$ using $\mathrm{Ni}_{0.2} \mathrm{Co}_{0.8} \mathrm{Se}$ and $\mathrm{Pt} / \mathrm{C}+\mathrm{RuO}_{2}$. (c) Polarization and power density curves of the $\mathrm{ZABs}$ using the $\mathrm{Ni}_{0.2} \mathrm{Co}_{0.8} \mathrm{Se}$ and $\mathrm{Pt} / \mathrm{C}+\mathrm{RuO}_{2}$ catalysts. (d) Schematic of the self-made all-solid-state $\mathrm{ZAB}$. (e) Cycling performance of the all-solid-state $\mathrm{ZAB}$ using $\mathrm{Ni}_{0.2} \mathrm{Co}_{0.8} \mathrm{Se}$ at $2 \mathrm{~mA} \mathrm{~cm}^{-2}$, where each cycle lasted $2 \mathrm{~min}$. (f) Charge-discharge polarization curves of the all-solid-state $\mathrm{ZAB}$ using Ni ${ }_{0.2} \mathrm{Co}_{0.8} \mathrm{Se}_{\text {. }}(\mathrm{g}$ ) Polarization and power density curves of the all-solid-state ZAB using $\mathrm{Ni}_{0.2} \mathrm{Co}_{0.8} \mathrm{Se}$. (h) The open-circuit voltage of an all-solid-state ZAB and (i) an LED circuit board powered by three all-solid-state ZABs. Reprinted with permission from Ref. [80]. Copyright 2019, Springer. 
and abundant active sites were generated homogenously that are advantageous for both ORR/OER and Faradaic mechanism. The generation of $\mathrm{NiOOH} / \mathrm{CoOOH}$ phase after full charge process could be presented as

$\mathrm{Co}(\mathrm{OH})_{2}+\mathrm{OH}^{-} \leftrightarrow \mathrm{CoOOH}+\mathrm{H}_{2} \mathrm{O}+\mathrm{e}^{-}$,

$\mathrm{Ni}(\mathrm{OH})_{2}+\mathrm{OH}^{-} \leftrightarrow \mathrm{NiOOH}+\mathrm{H}_{2} \mathrm{O}+\mathrm{e}^{-}$.

Using sodium poly-acrylate as electrolyte, the flexible hybrid battery provided a high energy density of $944.8 \mathrm{~W} \mathrm{~h} \mathrm{~kg}^{-1}$ and ultralong life span in both open-air and tightly sealed environments due to the combination of Ni/Co Faradaic redox reaction and OER/ORR mechanism, representing its environmental adaption and satisfactory durability. This approach can be extended for the fabrication of other BMC-based heterostructures that can efficiently improve the energy density of hybrid Zn batteries.

\section{RABs}

With rising demands for sustainable and renewable EES systems, $\mathrm{RAB}$ systems have stimulated attention due to their advantages over organic-based MIBs like higher safety, easy manufacturing, lower cost, higher ionic conductivity, and environmental benignity. These battery systems display higher energy density and power density compared with aqueous batteries, and bridge the gap between the MIBs and SCs. MIBs display the alkali metal insertion/extraction reaction mechanism accompanied by conversion in organic/aqueous electrolytes. However, in RABs using alkaline electrolytes, preferably $\mathrm{OH}^{-} / \mathrm{H}^{+}$insertion/extraction and conversion reaction mechanism prevail charge transfer between the electrolyte and active materials just like in SCs [266]. However, in SCs, only the surface electrochemical reactions are involved, but in RABs, both the surface and the bulk active materials take parts in the storage mechanism, and reactions mainly occur within the bulk of the active materials.

It is considered that the inadequacy of existing cathode materials with high performance inhibits their large-scale application; therefore exploring cathode materials is the key to develop the RABs. Recently, BMC-based materials have been revealed as potential electrode materials for RABs due to their superior conductivity, and synergistic effect of two different metal atoms that can provide multiple Faradaic redox reactions [120,186,227,267-269]. However, the electrochemical inactive phases, formation of unstable phases, oxidation of BMCs in alkaline electrolyte, and large volume expansion during reaction mechanisms are key challenges of cathode materials for RABs [266,268]. Various strategies like structural optimization, surface modification and hybridization in BMC-based cathode materials have been used to overcome these issues with the emphasis on enhancing the battery performance [227,267-269]. For example, a hierarchical nanostructure of uniform dendritic $\mathrm{NiCo}_{2} \mathrm{~S}_{4} @$ $\mathrm{NiCo}_{2} \mathrm{~S}_{4}$ nanoarrays with the width of $100 \mathrm{~nm}$ was designed as a cathode for the RAB system, and exhibited a high specific capacity $\left(4.43 \mathrm{~mA} \mathrm{~h} \mathrm{~cm}^{-2}\right)$ at a high current density $\left(240 \mathrm{~mA} \mathrm{~cm}^{-2}\right)$, outstanding rate performance and high cyclic stability [270]. The redox reaction observed in $\mathrm{NiCo}_{2} \mathrm{~S}_{4}$-based electrodes upon electrochemical reactions can be expressed as

$\mathrm{CoS}+\mathrm{OH}^{-} \leftrightarrow \mathrm{CoSOH}+\mathrm{e}^{-}$,

$\mathrm{CoSOH}+\mathrm{OH}^{-} \leftrightarrow \mathrm{CoSO}+\mathrm{H}_{2} \mathrm{O}+\mathrm{e}^{-}$,

$\mathrm{NiS}+\mathrm{OH}^{-} \leftrightarrow \mathrm{NiSOH}+\mathrm{e}^{-}$.
The excellent electrochemical performance in $\mathrm{NiCo}_{2} \mathrm{~S}_{4} @$ $\mathrm{NiCo}_{2} \mathrm{~S}_{4}$-based cathode was attributed to the presence of void space between the ravine-like nanosheet, providing the spatial confinement for electrolyte diffusion and penetration, porous hierarchical structure accommodating the volume expansion, and large specific surface area with large electric double-layer capacitance [270]. Similarly, various Ni-Co-S-based cathodes such as hollow $\mathrm{NiCo}_{2} \mathrm{~S}_{4}$ nanospheres [227], S,N co-doped graphene@CoNi $\mathrm{S}_{4}$ aerogels [268], N,S-carbon quantum dots@Ni$\mathrm{Co}_{2} \mathrm{~S}_{4}$ [267], and yolk-shell $\mathrm{Ni}-\mathrm{Mo}-\mathrm{Co}-\mathrm{S}$ nanocage arrays on $\mathrm{NF}$ [269], have been designed and synthesized for RAB systems.

Besides Ni-Co-S, other BMCs have also been explored by researchers for RAB systems $[87,120,186]$. For example, Shen et al. [120] reported a $\mathrm{KCu}_{7} \mathrm{~S}_{4} / \mathrm{rGO}$ composite negative electrode material for high-performance $\mathrm{RAB}$ and observed the charge storage mechanism and structural evolution in tunnel structured $\mathrm{KCu}_{7} \mathrm{~S}_{4}$. They found that the charge storage mechanism could be ascribed to the oxidation of $\mathrm{Cu}^{+}$to $\mathrm{Cu}^{2+}$ and $\mathrm{Cu}^{3+}$, and good reversible redox reactions. However, upon long charging/discharging cycles, the electrochemical induced irreversible phase transformation (i.e., from $\mathrm{Cu}_{7} \mathrm{~S}_{4}$ into $\mathrm{Cu}_{1.96} \mathrm{~S}$ ) is the main responsible factor in degrading the capacity of $\mathrm{KCu}_{7} \mathrm{~S}_{4}$ electrode. These intriguing findings propose the rational design and coupling of anode and cathode for developing high-performance RAB systems for practical applications.

\section{SUMMARY AND PERSPECTIVE}

This article provides a comprehensive overview of the recent development in the rational design and effective synthetic strategies of diverse BMCs and their nanohybrids with tuned shapes, sizes, morphologies, chemical compositions, and diverse nanoarchitectures, and illustrated their potential applications as electrode materials in various EES systems, including SCs, LIBs, SIBs, KIBs, multi-IBs, and MABs. Many inherent unique properties in BMCs such as higher electronic conductivity than mono metal chalcogenide counterparts, diverse chemical compositions, abundant redox chemistry, and synergic effects of multi-metal species endow them with excellent electrochemical activities. Further, various engineering strategies including structural, and nanohybrid engineering with carbonaceous materials, 3D templates, and metal compounds have been broadly developed to accelerate the electrochemical activities of BMCs for EES systems, and so the advantageous effects of these engineering strategies on the electrochemical performance are highlighted in this review article. In particular, morphology-controllable BMCbased electrode materials possess high surface area, small grain size, and large internal void space, which lead to a short ion diffusion path, facilitate large contact area between the electrolytes and active materials, and effectively accommodate the structural variation during the electrochemical performance. In contrast, incorporation of carbonaceous materials including pyrolytic carbon, rGO, CNTs, CNFs, with BMCs can not only further promote the electron/ion transport/diffusion, enhance electrochemical active sites, provide the effective buffering matrix to alleviate the huge volume variation of active materials during long cycling, but also decrease the agglomeration of nanoscale BMCs during charging/discharge processes and synthesis. Besides, the decoration of nanostructured BMCs arrays on 3D conductive templates such as CNT sponge, carbon foam, graphene foam, $\mathrm{CC}$ and $\mathrm{NF}$, provides 3D interconnected and straightforward paths for electron/ion and the larger contact 
area between active materials and electrolyte, eliminates the dead elements, and enables a flexible electrode for the wearable electronic application. Additionally, a wide range of capacitive materials has been incorporated with BMCs, generating heterostructured materials to improve the capacitive performance. Significantly, these resultant BMC materials achieved from various engineering strategies present a remarkable performance in various EES systems. Specifically, when evaluated as electrode materials for MIBs and SCs, high capacity/capacitance, improved cycling stability, enhanced rate performance, and energy and power densities were obtained by the BMCs. As for MABs, BMCs showed excellent electrocatalytic activities for ORR and OER.

In spite of lots of studies that have been done in this research area, many challenges still remain in the rational design of BMCbased nanostructured materials in a highly controllable fashion, and as well as in the fundamental understanding of charge storage mechanisms in BMC-based electrodes. The main challenges and future research direction associated with BMC-based electrode materials in EES are presented as follows:

(1) Nanostructured BMCs confined in hollow structures offer rich electroactive sites, high local conductivity and large interior space, therefore boosting the performance of EES systems. Till now, diverse BMCs confined in hollow structures have been fabricated; however, this research is still in its preliminary stage, and further innovative confined hollow nano/micro-structures with controlled configurations, diverse chemical compositions, and multifunctional properties with improved electrochemical properties need to be explored.

(2) The electrochemical performance of BMC-based composites obtained from hybrid engineering not only depends on the morphology, shapes and sizes, crystal growth, crystal structure, and their components, but also is related to spatial organization, exposed surface area, distribution and physical interaction between different components. For instance, the BMCs are directly decorated with/on the pyrolytic carbon, and BMC nanoparticles are also embedded in the pyrolytic carbon matrix. Though they are composed of similar components, better electrochemical performance is achieved with the composite in which BMCs are embedded with pyrolytic carbon than the other two. Therefore, it is very important to study the spatial organization, interaction and distribution of components in the composites to get high electrochemical performance.

(3) Although BMC materials obtained from nanostructure engineering might provide rapid reaction kinetics with improved electrochemical performance to some extent, side reactions and low tap density might be major issues. The pack density and unwanted reactions can be alleviated by designing a 3D trilayer electrode in which active materials (MBCs) can be sandwiched between an inner carbon framework (core) and an outer amorphous carbon layer. The pack density can also be improved by using BMCs (active materials) with a secondary structure, such as micro-nanostructures.

(4) In EES systems, most of the studies of BMCs focused on the SCs and LIBs/SIBs, but little attention has been given to PIBs and multi-IBs. For PIBs or multi-IBs, the BMC-based electroactive materials showed inferior cycling stability and poor rate performance. Therefore, more attention is required to explore the failure mechanisms in electrode materials during long-term charging/discharging.

(5) Most of the reported performance values of BMC electroactive materials in EES systems are based on individual observations. To better understand and to make the standard, a comprehensive study is required. The combination of experimental, theoretical simulation and mathematical modeling can be beneficial in modulating the design and structures, and properties of BMCs to improve the electrochemical performance.

(6) The control of structure at the atomic level can precisely and considerably modulate the physiochemical properties of various materials including nobel metals and metal oxides, and has shown big achievement in the field of energy conversion and storage $[97,271]$. The atomic level engineering not only changes the charge storage mechanism of oxide materials from surface redox to intercalation into bulk materials, but also enhances the electronic and ionic conductivity [97]. This atomic-level engineering approach can also be applied in BMC-based electrode materials to design future energy storage devices with high energy density and power density. However, synthesis and precise control at the atomic level is a quite challenging process. Thus optimization in experiments, the relationship between the precise structure of BMCs and properties, and advanced characterizations at each engineering stage are indispensable to achieve the best electrochemical performances.

(7) The practical application of BMCs has been prevented due to abnormal voltage hysteresis between discharge/charge steps. This large hysteresis can be alleviated by optimizing the material microstructure and electrode structure design. However, most of the published studies of BMCs have concentrated only on their synthesis and traditional electrochemical testing, but less attention has been given to its physical origin. To overcome the hysteresis issue, it is essential to understand its origin that has been remained elusive.

(8) The electrochemical reactions involved in EES devices are complex and may cause severe morphological/structural changes in electroactive materials. An in-depth understanding of the fundamental mechanism involved in novel electroactive materials based on BMCs during the real-time electrochemical process is also very important to develop high-performance EES devices. Therefore, in-situ characterization techniques such as XRD, Raman spectroscopy, XPS, TEM, AFM, can be more beneficial in examining the morphological changes, microstructure/phase transformation, ion diffusion and electrode/ electrolyte interfaces at various length scales and high temporal resolution during the electrochemical process. The basic information from in-situ characterizations can propose important understandings for designing structural and compositional modification approaches in improving the performance of EES devices.

Received 24 September 2021; accepted 9 November 2021; published online 31 December 2021

1 Su Q, Du G, Zhang J, et al. In situ transmission electron microscopy observation of electrochemical sodiation of individual $\mathrm{Co}_{9} \mathrm{~S}_{8}$-filled carbon nanotubes. ACS Nano, 2014, 8: 3620-3627

2 Chen S, Feng Y, Wang J, et al. Free-standing N-doped hollow carbon fibers as high-performance anode for potassium ion batteries. Sci China Mater, 2020, 64: 547-556

3 Chen X, Ji H, Chen W, et al. In situ protection of a sulfur cathode and a lithium anode via adopting a fluorinated electrolyte for stable lithium-sulfur batteries. Sci China Mater, 2021, 64: 2127-2138

4 Han S, Chen Y, Hao Y, et al. Multi-dimensional hierarchical $\mathrm{CoS}_{2} @$ MXene as trifunctional electrocatalysts for zinc-air batteries and 
overall water splitting. Sci China Mater, 2020, 64: 1127-1138

5 Jiang S, Yun S, Cao H, et al. Porous carbon matrix-encapsulated $\mathrm{MnO}$ in situ derived from metal-organic frameworks as advanced anode materials for Li-ion capacitors. Sci China Mater, 2022, 65: 59-68

6 Jin J, Liu Y, Pang X, et al. A comprehensive understanding of the anionic redox chemistry in layered oxide cathodes for sodium-ion batteries. Sci China Chem, 2021, 64: 385-402

7 Kong L, Liu Y, Huang $\mathrm{H}$, et al. Interconnected $\mathrm{CoS}_{2} / \mathrm{NC}$-CNTs network as high-performance anode materials for lithium-ion batteries. Sci China Mater, 2020, 64: 820-829

8 Li G, Mu Y, Huang Z, et al. Poly-active centric $\mathrm{Co}_{3} \mathrm{O}_{4}-\mathrm{CeO}_{2} / \mathrm{Co}-\mathrm{N}-\mathrm{C}$ composites as superior oxygen reduction catalysts for $\mathrm{Zn}$-air batteries. Sci China Mater, 2020, 64: 73-84

9 Chen Y, Wang Y, Wang Z, et al. Densification by compaction as an effective low-cost method to attain a high areal lithium storage capacity in a CNT@ $\mathrm{Co}_{3} \mathrm{O}_{4}$ sponge. Adv Energy Mater, 2018, 8: 1702981

10 Shuai H, Li J, Jiang F, et al. Electrochemically intercalated intermediate induced exfoliation of few-layer $\mathrm{MoS}_{2}$ from molybdenite for long-life sodium storage. Sci China Mater, 2020, 64: 115-127

11 Wang $\mathrm{H}$, Zhu J, Su Y, et al. Interfacial compatibility issues in rechargeable solid-state lithium metal batteries: A review. Sci China Chem, 2021, 64: 879-898

$12 \mathrm{Xu} \mathrm{J}, \mathrm{Xu} \mathrm{Y}$, Lai $\mathrm{C}$, et al. Challenges and perspectives of covalent organic frameworks for advanced alkali-metal ion batteries. Sci China Chem, 2021, 64: 1267-1282

13 Zhang SZ, Zhang XW. A comparative study of different online model parameters identification methods for lithium-ion battery. Sci China Technol Sci, 2021, 64: 2312-2327

$14 \mathrm{Li} \mathrm{W}$, Zeng L, Wu Y, et al. Nanostructured electrode materials for lithium-ion and sodium-ion batteries via electrospinning. Sci China Mater, 2016, 59: 287-321

15 Li Y, Zhao Y, Feng X, et al. A durable P2-type layered oxide cathode with superior low-temperature performance for sodium-ion batteries. Sci China Mater, 2022, 65:

16 Massé RC, Uchaker E, Cao G. Beyond Li-ion: Electrode materials for sodium- and magnesium-ion batteries. Sci China Mater, 2015, 58: 715-766

17 Mahmood A, Li S, Ali Z, et al. Ultrafast sodium/potassium-ion intercalation into hierarchically porous thin carbon shells. Adv Mater, 2019, 31: 1805430

18 Li S, Hua M, Yang Y, et al. Phosphorous-doped bimetallic sulfides embedded in heteroatom-doped carbon nanoarrays for flexible allsolid-state supercapacitors. Sci China Mater, 2021, 64: 2439-2453

19 Meng A, Shen T, Huang T, et al. $\mathrm{NiCoSe}_{2} / \mathrm{Ni}_{3} \mathrm{Se}_{2}$ lamella arrays grown on $\mathrm{N}$-doped graphene nanotubes with ultrahigh-rate capability and long-term cycling for asymmetric supercapacitor. Sci China Mater, 2019, 63: 229-239

20 Yousaf $\mathrm{M}$, Wang $\mathrm{Y}$, Chen $\mathrm{Y}$, et al. A 3D trilayered CNT/MoSe $/ \mathrm{C}$ heterostructure with an expanded $\mathrm{MoSe}_{2}$ interlayer spacing for an efficient sodium storage. Adv Energy Mater, 2019, 9: 1900567

21 Fang G, Wang Q, Zhou J, et al. Metal organic framework-templated synthesis of bimetallic selenides with rich phase boundaries for sodium-ion storage and oxygen evolution reaction. ACS Nano, 2019, 13: 5635-5645

22 Yousaf M, Mahmood A, Wang Y, et al. Advancement in layered transition metal dichalcogenide composites for lithium and sodium ion batteries. JEE, 2016, 4: 58

23 Wang Y, Ma Z, Chen Y, et al. Controlled synthesis of core-shell carbon@ $\mathrm{MoS}_{2}$ nanotube sponges as high-performance battery electrodes. Adv Mater, 2016, 28: 10175-10181

24 Yu XY, David Lou XW. Mixed metal sulfides for electrochemical energy storage and conversion. Adv Energy Mater, 2018, 8: 1701592

25 Fang Y, Luan D, Lou XWD. Recent advances on mixed metal sulfides for advanced sodium-ion batteries. Adv Mater, 2020, 32: 2002976

26 Yuan C, Wu HB, Xie Y, et al. Mixed transition-metal oxides: Design, synthesis, and energy-related applications. Angew Chem Int Ed, 2014, 53: $1488-1504$

27 Shen J, Ji J, Dong P, et al. Novel $\mathrm{FeNi}_{2} \mathrm{~S}_{4} / \mathrm{TMD}$-based ternary composites for supercapacitor applications. J Mater Chem A, 2016, 4:
$8844-8850$

28 Li L, Jacobs R, Gao P, et al. Origins of large voltage hysteresis in highenergy-density metal fluoride lithium-ion battery conversion electrodes. J Am Chem Soc, 2016, 138: 2838-2848

29 Fu G, Lee JM. Ternary metal sulfides for electrocatalytic energy conversion. J Mater Chem A, 2019, 7: 9386-9405

30 Chen X, Liu Q, Bai T, et al. Nickel and cobalt sulfide-based nanostructured materials for electrochemical energy storage devices. Chem Eng J, 2021, 409: 127237

31 Ali Z, Zhang T, Asif M, et al. Transition metal chalcogenide anodes for sodium storage. Mater Today, 2020, 35: 131-167

32 Xie D, Xia X, Zhong Y, et al. Exploring advanced sandwiched arrays by vertical graphene and $\mathrm{N}$-doped carbon for enhanced sodium storage. Adv Energy Mater, 2017, 7: 1601804

33 Wang W, Zhu X, Zhang Y, et al. Structural designs for accommodating volume expansion in sodium ion batteries. Chin J Chem, 2018, 36: 866-874

34 Fang Y, Yu XY, Lou XWD. Nanostructured electrode materials for advanced sodium-ion batteries. Matter, 2019, 1: 90-114

$35 \mathrm{Du} \mathrm{W}$, Zhu Z, Wang Y, et al. One-step synthesis of $\mathrm{CoNi}_{2} \mathrm{~S}_{4}$ nanoparticles for supercapacitor electrodes. RSC Adv, 2014, 4: 6998

36 Li H, Li Z, Sun M, et al. Zinc cobalt sulfide nanoparticles as high performance electrode material for asymmetric supercapacitor. Electrochim Acta, 2019, 319: 716-726

37 Tang J, Ge Y, Shen J, et al. Facile synthesis of $\mathrm{CuCo}_{2} \mathrm{~S}_{4}$ as a novel electrode material for ultrahigh supercapacitor performance. Chem Commun, 2016, 52: 1509-1512

38 Hussain $\mathrm{S}$, Liu T, Aslam N, et al. Truncated $\mathrm{NiCo}_{2} \mathrm{~S}_{4}$ cubohexa-octahedral nanostructures for high-performance supercapacitor. Mater Lett, 2017, 189: 21-24

39 Bezverkhyy I, Danot M, Afanasiev P. New low-temperature preparations of some simple and mixed $\mathrm{Co}$ and $\mathrm{Ni}$ dispersed sulfides and their chemical behavior in reducing atmosphere. Inorg Chem, 2003, 42: 1764-1768

40 Wiltrout AM, Read CG, Spencer EM, et al. Solution synthesis of thiospinel $\mathrm{CuCo}_{2} \mathrm{~S}_{4}$ nanoparticles. Inorg Chem, 2016, 55: 221-226

$41 \mathrm{Yu} \mathrm{Z}$, Tetard L, Zhai L, et al. Supercapacitor electrode materials: Nanostructures from 0 to 3 dimensions. Energy Environ Sci, 2015, 8: 702-730

42 Ramasamy K, Gupta RK, Palchoudhury S, et al. Layer-structured copper antimony chalcogenides $\left(\mathrm{CuSbSe}_{x} \mathrm{~S}_{2-x}\right)$ : Stable electrode materials for supercapacitors. Chem Mater, 2014, 27: 379-386

43 Bhattacharjya D, Sinhamahapatra A, Ko JJ, et al. High capacity and exceptional cycling stability of ternary metal sulfide nanorods as $\mathrm{Li}$ ion battery anodes. Chem Commun, 2015, 51: 13350-13353

44 Sun $\mathrm{S}$, Nie Y, Sun M, et al. Facile synthesis of $\mathrm{CoNi}_{2} \mathrm{~S}_{4}$ one-dimensional nanorods as anode for high performance lithium ion batteries. Mater Lett, 2016, 176: 87-90

45 Li XL, Bai S, Yue XY, et al. Rod-shaped monoclinic $\mathrm{CoMo}_{2} \mathrm{~S}_{4}$ with exceptionally reversible phase conversion for sodium storage. J Alloys Compd, 2020, 838: 155613

46 Tang Y, Chen T, Yu S, et al. A highly electronic conductive cobalt nickel sulphide dendrite/quasi-spherical nanocomposite for a supercapacitor electrode with ultrahigh areal specific capacitance. J Power Sources, 2015, 295: 314-322

47 Ramasamy K, Gupta RK, Sims H, et al. Layered ternary sulfide $\mathrm{CuSbS}_{2}$ nanoplates for flexible solid-state supercapacitors. J Mater Chem A, 2015, 3: 13263-13274

48 Chen $\mathrm{W}$, Chen $\mathrm{H}$, Zhu $\mathrm{H}$, et al. Solvothermal synthesis of ternary $\mathrm{Cu}_{2} \mathrm{MoS}_{4}$ nanosheets: Structural characterization at the atomic level. Small, 2014, 10: 4637-4644

49 Khalafallah D, Wu Z, Zhi M, et al. Rational design of porous structured nickel manganese sulfides hexagonal sheets-in-cage structures as an advanced electrode material for high-performance electrochemical capacitors. Chem Eur J, 2020, 26: 2251-2262

50 Zhang Q, Wang L, Wang J, et al. Semimetallic vanadium molybdenum sulfide for high-performance battery electrodes. J Mater Chem A 2018, 6: 9411-9419

51 Balaji R, Balamurugan J, Nguyen TT, et al. Tunable construction of 
$\mathrm{Fe}_{x} \mathrm{Co}_{3-x} \mathrm{Se}_{4}$ nanostructures as advanced electrode for boosting capacity and energy density. Chem Eng J, 2020, 390: 124557

52 Crossland CJ, Hickey PJ, Evans JSO. The synthesis and characterisation of $\mathrm{Cu}_{2} \mathrm{MX}_{4}(\mathrm{M}=\mathrm{W}$ or $\mathrm{Mo} ; \mathrm{X}=\mathrm{S}$, Se or $\mathrm{S} / \mathrm{Se})$ materials prepared by a solvothermal method. J Mater Chem, 2005, 15: 3452

53 Tran PD, Nguyen M, Pramana SS, et al. Copper molybdenum sulfide: A new efficient electrocatalyst for hydrogen production from water. Energy Environ Sci, 2012, 5: 8912

54 Hu X, Shao W, Hang X, et al. Superior electrical conductivity in hydrogenated layered ternary chalcogenide nanosheets for flexible allsolid-state supercapacitors. Angew Chem Int Ed, 2016, 55: 5733-5738

55 Shen L, Yu L, Wu HB, et al. Formation of nickel cobalt sulfide ball-inball hollow spheres with enhanced electrochemical pseudocapacitive properties. Nat Commun, 2015, 6: 6694

56 Chen J, Zhang L, Bai W, et al. Unique hollow-concave $\mathrm{CoMoS}_{x}$ boxes with abundant mesoporous structure for high-performance hybrid supercapacitors. Electrochim Acta, 2020, 337: 135824

57 Hou L, Bao R, Rehan M, et al. Uniform hollow mesoporous nickel cobalt sulfide microdumbbells: A competitive electrode with exceptional gravimetric/volumetric pseudocapacitance for high-energydensity hybrid superapacitors. Adv Electron Mater, 2017, 3: 1600322

58 Jin $\mathrm{Y}, \mathrm{Xu} \mathrm{J}$, Wang $\mathrm{L}$, et al. Template-free synthesis of nanorod-assembled hierarchical $\mathrm{Zn}_{1-x} \mathrm{Mn}_{x} \mathrm{~S}$ hollow nanostructures with enhanced pseudocapacitive properties. Chem Eur J, 2016, 22: 18859-18864

59 Qu G, Zhang X, Xiang G, et al. ZIF-67 derived hollow Ni-Co-Se nanopolyhedrons for flexible hybrid supercapacitors with remarkable electrochemical performances. Chin Chem Lett, 2020, 31: 2007-2012

60 Chen J, Mohrhusen L, Ali G, et al. Electrochemical mechanism investigation of $\mathrm{Cu}_{2} \mathrm{MoS}_{4}$ hollow nanospheres for fast and stable sodium ion storage. Adv Funct Mater, 2019, 29: 1807753

61 Zardkhoshoui AM, Davarani SSH. Construction of complex coppercobalt selenide hollow structures as an attractive battery-type electrode material for hybrid supercapacitors. Chem Eng J, 2020, 402: 126241

62 Gai Y, Xie T, Shang Y, et al. Self-sacrificing template-derived hollowstructured $\mathrm{NiCo}_{2} \mathrm{~S}_{4}$ spheres with highly efficient supercapacitance performance. Energy Fuels, 2020, 34: 10203-10210

63 Mohammadi A, Moosavifard SE, Goljanian Tabrizi A, et al. Nanoporous $\mathrm{CuCo}_{2} \mathrm{~S}_{4}$ microspheres: A novel positive electrode for highperformance hybrid energy storage devices. ACS Appl Energy Mater, 2018, 2: 627-635

64 Liu YL, Yan C, Wang GG, et al. Selenium-rich nickel cobalt bimetallic selenides with core-shell architecture enable superior hybrid energy storage devices. Nanoscale, 2020, 12: 4040-4050

65 Zhang L, Zhang $\mathrm{H}$, Chu X, et al. Synthesis of size-controllable $\mathrm{NiCo}_{2} \mathrm{~S}_{4}$ hollow nanospheres toward enhanced electrochemical performance. Energy Environ Mater, 2020, 3: 421-428

66 Li J, Liu B, Li M, et al. Engineering $\mathrm{Zn}_{0.33} \mathrm{Co}_{0.67} \mathrm{~S}$ hollow microspheres with enhanced electrochemical performance for lithium and sodium ion batteries. Eur J Inorg Chem, 2018, 2018(26): 3036-3040

67 Tavakoli F, Rezaei B, Taghipour Jahromi AR, et al. Facile synthesis of yolk-shelled $\mathrm{CuCo}_{2} \mathrm{Se}_{4}$ microspheres as a novel electrode material for supercapacitor application. ACS Appl Mater Interfaces, 2020, 12: 418427

68 Zhu T, Wang J, Ho GW. Self-supported yolk-shell nanocolloids towards high capacitance and excellent cycling performance. Nano Energy, 2015, 18: 273-282

69 Xia C, Alshareef HN. Self-templating scheme for the synthesis of nanostructured transition-metal chalcogenide electrodes for capacitive energy storage. Chem Mater, 2015, 27: 4661-4668

70 Mohammadi Zardkhoshoui A, Ameri B, Hosseiny Davarani SS. A high-energy-density supercapacitor with multi-shelled nickel-manganese selenide hollow spheres as cathode and double-shell nickel-iron selenide hollow spheres as anode electrodes. Nanoscale, 2021, 13: 2931-2945

$71 \mathrm{Yu} \mathrm{L}$, Zhang L, Wu HB, et al. Formation of $\mathrm{Ni}_{x} \mathrm{Co}_{3-x} \mathrm{~S}_{4}$ hollow nanoprisms with enhanced pseudocapacitive properties. Angew Chem Int Ed, 2014, 53: 3711-3714

72 Wan $\mathrm{H}$, Jiang $\mathrm{J}, \mathrm{Yu}$ J, et al. $\mathrm{NiCo}_{2} \mathrm{~S}_{4}$ porous nanotubes synthesis via sacrificial templates: High-performance electrode materials of super- capacitors. CrystEngComm, 2013, 15: 7649

73 Li M, Li X, Li Z, et al. Retraction of "hierarchical nanosheet-built $\mathrm{CoNi}_{2} \mathrm{~S}_{4}$ nanotubes coupled with carbon-encapsulated carbon nanotubes@ $\mathrm{Fe}_{2} \mathrm{O}_{3}$ composites toward high-performance aqueous hybrid supercapacitor devices". ACS Appl Mater Interfaces, 2019, 11: 29476

74 Le LT, Truong DQ, Ung TTD, et al. $\mathrm{Cu}_{2} \mathrm{MoS}_{4}$ nanotubes as a cathode material for rechargeable magnesium-ion battery. ChemistrySelect, 2020, 5: 280-283

75 Guan BY, Yu L, Wang X, et al. Formation of onion-like $\mathrm{NiCo}_{2} \mathrm{~S}_{4}$ particles via sequential ion-exchange for hybrid supercapacitors. Adv Mater, 2017, 29: 1605051

76 Tang Y, Chen S, Mu S, et al. Synthesis of capsule-like porous hollow nanonickel cobalt sulfides via cation exchange based on the Kirkendall effect for high-performance supercapacitors. ACS Appl Mater Interfaces, 2016, 8: 9721-9732

77 Chen YM, Li Z, Lou XWD. General formation of $\mathrm{M}_{x} \mathrm{Co}_{3-x} \mathrm{~S}_{4}(\mathrm{M}=\mathrm{Ni}$, $\mathrm{Mn}, \mathrm{Zn}$ ) hollow tubular structures for hybrid supercapacitors. Angew Chem Int Ed, 2015, 54: 10521-10524

78 Miao C, Zhou C, Wang HE, et al. Hollow Co-Mo-Se nanosheet arrays derived from metal-organic framework for high-performance supercapacitors. J Power Sources, 2021, 490: 229532

79 Zhang Y, Li T, Cao S, et al. $\mathrm{Cu}_{2} \mathrm{MoS}_{4}$ hollow nanocages with fast and stable $\mathrm{Mg}^{2+}$-storage performance. Chem Eng J, 2020, 387: 124125

80 Qian $\mathrm{Z}$, Chen $\mathrm{Y}$, Tang $\mathrm{Z}$, et al. Hollow nanocages of $\mathrm{Ni}_{x} \mathrm{Co}_{1-x} \mathrm{Se}$ for efficient zinc-air batteries and overall water splitting. Nano-Micro Lett, 2019, 11: 28

81 Hou L, Shi Y, Wu C, et al. Monodisperse metallic $\mathrm{NiCoSe}_{2}$ hollow sub-microspheres: Formation process, intrinsic charge-storage mechanism, and appealing pseudocapacitance as highly conductive electrode for electrochemical supercapacitors. Adv Funct Mater, 2018 , 28: 1705921

82 Song X, Huang C, Qin Y, et al. Hierarchical hollow, sea-urchin-like and porous $\mathrm{Ni}_{0.5} \mathrm{Co}_{0.5} \mathrm{Se}_{2}$ as advanced battery material for hybrid supercapacitors. J Mater Chem A, 2018, 6: 16205-16212

83 Cheng L, Chen S, Zhang Q, et al. Hierarchical sea-urchin-like bimetallic zinc-cobalt selenide for enhanced battery-supercapacitor hybrid device. J Energy Storage, 2020, 31: 101663

84 Cheng L, Zhai Q, Chen S, et al. Component-tunable hierarchical flower-shaped bimetallic zinc-cobalt selenides for high-performance hybrid supercapacitor. J Energy Storage, 2021, 36: 102374

85 Dou S, Xu J, Sari HMK, et al. Large interlayer spacing of few-layered cobalt-tin-based sulfide providing superior sodium storage. ACS Appl Mater Interfaces, 2020, 12: 41546-41556

86 Yan D, Huang S, Von Lim Y, et al. Stepwise intercalation-conversionintercalation sodiation mechanism in $\mathrm{CuInS}_{2}$ prompting sodium storage performance. ACS Energy Lett, 2020, 5: 3725-3732

87 Zhu Y, Li J, Yun X, et al. Hydrothermal synthesis of nanoflake-assembled $\left(\mathrm{Ni}_{0.5} \mathrm{Co}_{0.5}\right)_{0.85} \mathrm{Se}$ microspheres as the cathode and reduced graphene oxide/porous $\mathrm{Fe}_{2} \mathrm{O}_{3}$ nanospheres composite as the anode for novel alkaline aqueous batteries. ACS Sustain Chem Eng, 2020, 8 561-572

88 Zhao F, Xie D, Song X, et al. Construction of hydrangea-like nickel cobalt sulfide through efficient microwave-assisted approach for remarkable supercapacitors. Appl Surf Sci, 2021, 539: 148260

$89 \mathrm{Wu}$ J, Huang $\mathrm{X}$, Xia X. Exploring $\mathrm{NiCo}_{2} \mathrm{~S}_{4}$ nanosheets arrays by hydrothermal conversion for enhanced high-rate batteries. J Energy Chem, 2019, 35: 132-137

90 Zheng L, Song J, Ye X, et al. Construction of self-supported hierarchical NiCo-S nanosheet arrays for supercapacitors with ultrahigh specific capacitance. Nanoscale, 2020, 12: 13811-13821

91 Lim YV, Huang S, Wu Q, et al. Super kinetically pseudocapacitive $\mathrm{MnCo}_{2} \mathrm{~S}_{4}$ nanourchins toward high-rate and highly stable sodium-ion storage. Adv Funct Mater, 2020, 30: 1909702

92 Ren X, Du Y, Song M, et al. Facile preparation of mesoporous $\mathrm{NiCo}_{2} \mathrm{~S}_{4}$ microaggregates constructed by nanoparticles via puffing $\mathrm{NiCo}_{2} \mathrm{O}_{4}$ cubes for high performance asymmetric supercapacitors. J Alloys Compd, 2019, 806: 1481-1490

93 Ali Z, Asif M, Zhang T, et al. General approach to produce nanostructured binary transition metal selenides as high-performance so- 
dium ion battery anodes. Small, 2019, 15: 1901995

94 Zhong W, Ma Q, Tang W, et al. Construction of a bimetallic nickelcobalt selenide pompon used as a superior anode material for high performance sodium storage. Inorg Chem Front, 2020, 7: 1003-1011

95 Zhang $\mathrm{Y}$, Ma M, Yang J, et al. Shape-controlled synthesis of $\mathrm{NiCo}_{2} \mathrm{~S}_{4}$ and their charge storage characteristics in supercapacitors. Nanoscale, 2014, 6: 9824-9830

96 Gbadamasi S, Mohiuddin M, Krishnamurthi V, et al. Interface chemistry of two-dimensional heterostructures-Fundamentals to applications. Chem Soc Rev, 2021, 50: 4684-4729

97 Ling T, Da P, Zheng X, et al. Atomic-level structure engineering of metal oxides for high-rate oxygen intercalation pseudocapacitance. Sci Adv, 2018, 4: eaau6261

98 Wu S, Xue Y, Yang Q, et al. Conductive carbon spheres-supported nickel-cobalt selenide nanoparticles as a high-performance and longlife electrode for supercapacitors. Diamond Relat Mater, 2021, 111: 108187

99 Li L, Dai Z, Zhang Y, et al. Carbon@ $\mathrm{NiCo}_{2} \mathrm{~S}_{4}$ nanorods: An excellent electrode material for supercapacitors. RSC Adv, 2015, 5: 83408-83414

100 Liu X, Yang L, Xu G, et al. Pomegranate-like porous $\mathrm{NiCo}_{2} \mathrm{Se}_{4}$ spheres with $\mathrm{N}$-doped carbon as advanced anode materials for $\mathrm{Li} / \mathrm{Na}$-ion batteries. Green Energy Environ, 2020,

101 Zhang M, Liu Y, Zhang Y, et al. Bimetallic selenide $\mathrm{LiInSe}_{2}$ decorated with a uniform carbon layer with superior lithium storage performance. ChemElectroChem, 2019, 7: 139-147

102 Jia M, Jin Y, Zhao P, et al. Hollow $\mathrm{NiCoSe}_{2}$ microspheres@N-doped carbon as high-performance pseudocapacitive anode materials for sodium ion batteries. Electrochim Acta, 2019, 310: 230-239

103 Wei X, Zhang Y, Zhang B, et al. Yolk-shell-structured zinc-cobalt binary metal sulfide@ $\mathrm{N}$-doped carbon for enhanced lithium-ion storage. Nano Energy, 2019, 64: 103899

104 Zuo X, Song Y, Zhen M. Carbon-coated $\mathrm{NiCo}_{2} \mathrm{~S}_{4}$ multi-shelled hollow microspheres with porous structures for high rate lithium ion battery applications. Appl Surf Sci, 2020, 500: 144000

105 Hou BH, Wang YY, Liu DS, et al. $\mathrm{N}$-doped carbon-coated $\mathrm{Ni}_{1.8} \mathrm{Co}_{1.2-}$ $\mathrm{Se}_{4}$ nanoaggregates encapsulated in $\mathrm{N}$-doped carbon nanoboxes as advanced anode with outstanding high-rate and low-temperature performance for sodium-ion half/full batteries. Adv Funct Mater, 2018, 28: 1805444

106 Chu J, Yu Q, Yang D, et al. Thickness-control of ultrathin bimetallic Fe-Mo selenide@N-doped carbon core/shell "nano-crisps" for highperformance potassium-ion batteries. Appl Mater Today, 2018, 13: 344-351

107 Yang Y, Li S, Huang W, et al. Effective synthetic strategy for $\mathrm{Zn}_{0.76} \mathrm{Co}_{0.24} \mathrm{~S}$ encapsulated in stabilized $\mathrm{N}$-doped carbon nanoarchitecture towards ultra-long-life hybrid supercapacitors. J Mater Chem A, 2019, 7: 14670-14680

108 Ali Z, Asif M, Huang X, et al. Hierarchically porous $\mathrm{Fe}_{2} \mathrm{CoSe}_{4}$ binarymetal selenide for extraordinary rate performance and durable anode of sodium-ion batteries. Adv Mater, 2018, 30: 1802745

109 Yi M, Wu A, Chen Q, et al. In situ confined conductive nickel cobalt sulfoselenide with tailored composition in graphitic carbon hollow structure for energy storage. Chem Eng J, 2018, 351: 678-687

110 Wang MH, Guo SP. Highly uniform hollow $\mathrm{CuCo}_{2} \mathrm{~S}_{4} @ \mathrm{C}$ dodecahedra derived from ZIF-67 for high performance lithium-ion batteries. J Alloys Compd, 2020, 832: 154978

111 Li Z, Tian $\mathrm{M}$, Chen $\mathrm{Y}$, et al. MOFs derived $\left(\mathrm{Ni}_{0.75} \mathrm{Co}_{0.25}\right) \mathrm{Se}_{2}$ nanoparticles embedded in $\mathrm{N}$-doped nanocarbon for hybrid supercapacitors. Ceramics Int, 2021, 47: 12623-12630

112 Li L, Zhao J, Zhu Y, et al. Bimetallic Ni/Co-ZIF-67 derived $\mathrm{NiCo}_{2} \mathrm{Se}_{4} /$ $\mathrm{N}$-doped porous carbon nanocubes with excellent sodium storage performance. Electrochim Acta, 2020, 353: 136532

113 Zhou K, Tang D, Li W, et al. Synergetic lithium storage of bimetallic sulfide $\mathrm{Co}_{8} \mathrm{FeS}_{8} / \mathrm{N}-\mathrm{C}$ dodecahedral nanocages with enhanced lithiumion battery performance. Chem Eng Sci, 2019, 208: 115142

114 Fang $\mathrm{W}, \mathrm{Hu} \mathrm{H}$, Jiang $\mathrm{T}$, et al. $\mathrm{N}$ - and S-doped porous carbon decorated with in-situ synthesized $\mathrm{Co}-\mathrm{Ni}$ bimetallic sulfides particles: A cathode catalyst of rechargeable Zn-air batteries. Curr Alzheimer Resbon, 2019, 146: 476-485
115 Yang SH, Park SK, Park GD, et al. Conversion reaction mechanism of ultrafine bimetallic Co-Fe selenides embedded in hollow mesoporous carbon nanospheres and their excellent K-ion storage performance. Small, 2020, 16: 2002345

116 Yang SH, Park SK, Park GD, et al. Rational synthesis of uniform yolkshell Ni-Fe bimetallic sulfide nanoflakes@porous carbon nanospheres as advanced anodes for high-performance potassium-/sodium-ion batteries. Chem Eng J, 2021, 417: 127963

117 Qin J, Wang T, Liu D, et al. A top-down strategy toward SnSb in-plane nanoconfined 3D N-doped porous graphene composite microspheres for high performance Na-ion battery anode. Adv Mater, 2018, 30: 1704670

118 Xing W, Li X, Cai T, et al. Layered double hydroxides derived NiCosulfide as a cathode material for aluminum ion batteries. Electrochim Acta, 2020, 344: 136174

119 Jiao X, Hu X, Xi G, et al. $\mathrm{MnSb}_{2} \mathrm{~S}_{4}$ nanorods linked with interconnected reduced graphene oxide as high-performance anode for sodium ion batteries. Electrochim Acta, 2021, 366: 137317

120 Shen W, Zang J, Hu H, et al. Controlled synthesis of $\mathrm{KCu}_{7} \mathrm{~S}_{4} / \mathrm{rGO}$ nanocomposites for electrochemical energy storage. Mater Des, 2020 195: 108992

121 Moosavifard SE, Mohammadi A, Ebrahimnejad Darzi M, et al. A facile strategy to synthesis graphene-wrapped nanoporous copper-cobaltselenide hollow spheres as an efficient electrode for hybrid supercapacitors. Chem Eng J, 2021, 415: 128662

122 Liu X, Hao Y, Shu J, et al. Nitrogen/sulfur dual-doping of reduced graphene oxide harvesting hollow $\mathrm{ZnSnS}_{3}$ nano-microcubes with superior sodium storage. Nano Energy, 2019, 57: 414-423

123 Liu X, Ding S, Ye L, et al. Optimizing the supercapacitive performance via encasing MOF-derived hollow $(\mathrm{Ni}, \mathrm{Co}) \mathrm{Se}_{2}$ nanocubes into reduced graphene oxide. Chem Eng J, 2020, 399: 125789

124 Choi SH, Kang YC. Synergetic compositional and morphological effects for improved $\mathrm{Na}^{+}$storage properties of $\mathrm{Ni}_{3} \mathrm{Co}_{6} \mathrm{~S}_{8}$-reduced graphene oxide composite powders. Nanoscale, 2015, 7: 6230-6237

125 Peng $\mathrm{S}$, Li L, Li C, et al. In situ growth of $\mathrm{NiCo}_{2} \mathrm{~S}_{4}$ nanosheets on graphene for high-performance supercapacitors. Chem Commun, 2013, 49: 10178-10180

126 Yang J, Yu C, Fan X, et al. Electroactive edge site-enriched nickelcobalt sulfide into graphene frameworks for high-performance asymmetric supercapacitors. Energy Environ Sci, 2016, 9: 1299-1307

127 Nie L, Wang $\mathrm{H}$, Chai $\mathrm{Y}$, et al. In situ formation of flower-like $\mathrm{CuCo}_{2} \mathrm{~S}_{4}$ nanosheets/graphene composites with enhanced lithium storage properties. RSC Adv, 2016, 6: 38321-38327

128 Tao HC, Zhu SC, Yang XL, et al. Reduced graphene oxide decorated ternary $\mathrm{Cu}_{2} \mathrm{SnS}_{3}$ as anode materials for lithium ion batteries. J Electroanal Chem, 2016, 760: 127-134

129 Ye J, Chen T, Chen Q, et al. Facile hydrothermal synthesis of $\mathrm{SnCoS}_{4} /$ graphene composites with excellent electrochemical performance for reversible lithium ion storage. J Mater Chem A, 2016, 4: 13194-13202

130 Guo M, Balamurugan J, Thanh TD, et al. Facile fabrication of $\mathrm{Co}_{2} \mathrm{CuS}_{4}$ nanoparticle anchored $\mathrm{N}$-doped graphene for high-performance asymmetric supercapacitors. J Mater Chem A, 2016, 4: 17560-17571

131 Gong $\mathrm{Y}$, Zhao J, Wang $\mathrm{H}$, et al. $\mathrm{CuCo}_{2} \mathrm{~S}_{4} /$ reduced graphene oxide nanocomposites synthesized by one-step solvothermal method as anode materials for sodium ion batteries. Electrochim Acta, 2018, 292: 895-902

$132 \mathrm{Xu} \mathrm{H}$, Wang $\mathrm{C}$, Zhang J, et al. Rational design of bimetal-organic framework-derived $\mathrm{ZnSnS}_{3}$ nanodots incorporated into the nitrogendoped graphene framework for advanced lithium storage. ACS Sustain Chem Eng, 2020, 8: 4464-4473

133 Ghosh S, Samanta P, Murmu NC, et al. Investigation of electrochemical charge storage in nickel-cobalt-selenide/reduced graphene oxide composite electrode and its hybrid supercapacitor device. J Alloys Compd, 2020, 835: 155432

134 Peng Q, Hu X, Zeng T, et al. $\mathrm{FeSb}_{2} \mathrm{~S}_{4}$ anchored on amine-modified graphene towards high-performance anode material for sodium ion batteries. Chem Eng J, 2020, 385: 123857

135 Wang X, Zhao SX, Dong L, et al. One-step synthesis of surface-enriched nickel cobalt sulfide nanoparticles on graphene for high-per- 
formance supercapacitors. Energy Storage Mater, 2017, 6: 180-187

136

Zhang L, Huang Y, Miao YE, et al. Hierarchical composites of $\mathrm{NiCo}_{2} \mathrm{~S}_{4}$ nanorods grown on carbon nanofibers as anodes for high-performance lithium ion batteries. Compos Commun, 2020, 21: 100395

137 Yang $\mathrm{Z}, \mathrm{Wu} \mathrm{X}, \mathrm{Ma} \mathrm{C}$, et al. $\mathrm{Cu}_{2} \mathrm{SnSe}_{3} / \mathrm{CNT}$ composite as a promising anode material for sodium-ion batteries. Chem Res Chin Univ, 2020, 36: $91-96$

138 Zou J, Xie D, Zhao F, et al. Microwave rapid synthesis of nickel cobalt sulfides/CNTs composites as superior cycling ability electrode materials for supercapacitors. J Mater Sci, 2020, 56: 1561-1576

139 Luo Y, Que W, Yang C, et al. Hydrothermal synthesis of transition metal sulfides/MWCNT nanocomposites for high-performance asymmetric electrochemical capacitors. Electrochim Acta, 2019, 322: 134738

140 Mohanty US. Electrodeposition: A versatile and inexpensive tool for the synthesis of nanoparticles, nanorods, nanowires, and nanoclusters of metals. J Appl Electrochem, 2011, 41: 257-270

$141 \mathrm{Cao} \mathrm{X}, \mathrm{He} \mathrm{J}, \mathrm{Li} \mathrm{H}$, et al. $\mathrm{CoNi}_{2} \mathrm{~S}_{4}$ nanoparticle/carbon nanotube sponge cathode with ultrahigh capacitance for highly compressible asymmetric supercapacitor. Small, 2018, 14: 1800998

$142 \mathrm{Li} \mathrm{H}, \mathrm{Su} \mathrm{Y}$, Sun W, et al. Carbon nanotubes rooted in porous ternary metal sulfide@N/S-doped carbon dodecahedron: Bimetal-organicframeworks derivation and electrochemical application for high-capacity and long-life lithium-ion batteries. Adv Funct Mater, 2016, 26: 8345-8353

143 Xu J, Yang Y, Chu H, et al. Novel $\mathrm{NiCo}_{2} \mathrm{~S}_{4} @$ reduced graphene oxide@carbon nanotube nanocomposites for high performance supercapacitors. RSC Adv, 2016, 6: 100504-100510

144 Shen J, Tang J, Dong P, et al. Construction of three-dimensional $\mathrm{CuCo}_{2} \mathrm{~S}_{4} / \mathrm{CNT} /$ graphene nanocomposite for high performance supercapacitors. RSC Adv, 2016, 6: 13456-13460

145 Tong H, Bai W, Yue S, et al. Zinc cobalt sulfide nanosheets grown on nitrogen-doped graphene/carbon nanotube film as a high-performance electrode for supercapacitors. J Mater Chem A, 2016, 4: 1125611263

146 Huang X, Men S, Zheng H, et al. Highly porous $\mathrm{NiCoSe}_{4}$ microspheres as high-performance anode materials for sodium-ion batteries. Chem Asian J, 2020, 15: 1456-1463

147 Shen J, Xu X, Dong P, et al. Design and synthesis of three-dimensional needle-like $\mathrm{CoNi}_{2} \mathrm{~S}_{4} / \mathrm{CNT} /$ graphene nanocomposite with improved electrochemical properties. Ceramics Int, 2016, 42: 8120-8127

148 Zhou $\mathrm{C}$, Zhang $\mathrm{P}$, Liu J, et al. Hierarchical $\mathrm{NiCo}_{2} \mathrm{Se}_{4}$ nanoneedles/ nanosheets with $\mathrm{N}$-doped 3D porous graphene architecture as freestanding anode for superior sodium ion batteries. J Colloid Interface Sci, 2021, 587: 260-270

149 Yan S, Luo S, Feng J, et al. Rational design of flower-like $\mathrm{FeCo}_{2} \mathrm{~S}_{4} /$ reduced graphene oxide films: Novel binder-free electrodes with ultrahigh conductivity flexible substrate for high-performance all-solidstate pseudocapacitor. Chem Eng J, 2020, 381: 122695

$150 \mathrm{Li} \mathrm{G}, \mathrm{Xu}$ C. Hydrothermal synthesis of $3 \mathrm{D} \mathrm{Ni}_{x} \mathrm{Co}_{1-x} \mathrm{~S}_{2}$ particles/graphene composite hydrogels for high performance supercapacitors. Curr Alzheimer Resbon, 2015, 90: 44-52

151 Guo R, Dang L, Liu Z, et al. Incorporation of electroactive $\mathrm{NiCo}_{2} \mathrm{~S}_{4}$ and $\mathrm{Fe}_{2} \mathrm{O}_{3}$ into graphene aerogel for high-energy asymmetric supercapacitor. Colloids Surfs A-Physicochem Eng Aspects, 2020, 602: 125110

152 Hao P, Tian J, Sang Y, et al. 1D Ni-Co oxide and sulfide nanoarray/ carbon aerogel hybrid nanostructures for asymmetric supercapacitors with high energy density and excellent cycling stability. Nanoscale, 2016, 8: 16292-16301

153 Yousaf M, Wang Y, Chen Y, et al. Tunable free-standing core-shel CNT@MoSe $e_{2}$ anode for lithium storage. ACS Appl Mater Interfaces, 2018, 10: 14622-14631

154 Yousaf $\mathrm{M}$, Wang Z, Wang $\mathrm{Y}$, et al. Core-shell $\mathrm{FeSe}_{2} / \mathrm{C}$ nanostructures embedded in a carbon framework as a free standing anode for a sodium ion battery. Small, 2020, 16: 2002200

155 Yousaf $\mathrm{M}$, Chen $\mathrm{Y}$, Tabassum $\mathrm{H}$, et al. A dual protection system for heterostructured 3D CNT/CoSe $/ 2$ as high areal capacity anode for sodium storage. Adv Sci, 2020, 7: 1902907
156 Yousaf M, Shi HTH, Wang Y, et al. Novel pliable electrodes for flexible electrochemical energy storage devices: Recent progress and challenges. Adv Energy Mater, 2016, 6: 1600490

157 Chen J, Xu J, Zhou S, et al. Nitrogen-doped hierarchically porous carbon foam: A free-standing electrode and mechanical support for high-performance supercapacitors. Nano Energy, 2016, 25: 193-202

158 Shen L, Wang J, Xu G, et al. $\mathrm{NiCo}_{2} \mathrm{~S}_{4}$ nanosheets grown on nitrogendoped carbon foams as an advanced electrode for supercapacitors. Adv Energy Mater, 2015, 5: 1400977

159 Hua $\mathrm{M}$, Cui F, Huang $\mathrm{Y}$, et al. Crafting nanosheet-built $\mathrm{MnCo}_{2} \mathrm{~S}_{4}$ disks on robust $\mathrm{N}$-doped carbon matrix for hybrid supercapacitors. Electrochim Acta, 2019, 323: 134770

$160 \mathrm{Wu} \mathrm{X}, \mathrm{Li} \mathrm{S}$, Wang $\mathrm{B}$, et al. $\mathrm{NiCo}_{2} \mathrm{~S}_{4}$ nanotube arrays grown on flexible nitrogen-doped carbon foams as three-dimensional binder-free in tegrated anodes for high-performance lithium-ion batteries. Phys Chem Chem Phys, 2016, 18: 4505-4512

161 Khani H, Wipf DO. Iron oxide nanosheets and pulse-electrodeposited $\mathrm{Ni}$-Co-S nanoflake arrays for high-performance charge storage. ACS Appl Mater Interfaces, 2017, 9: 6967-6978

162 Chen W, Xia C, Alshareef HN. One-step electrodeposited nickel cobalt sulfide nanosheet arrays for high-performance asymmetric supercapacitors. ACS Nano, 2014, 8: 9531-9541

163 Sun $\mathrm{T}$, Huang $\mathrm{C}$, Shu $\mathrm{H}$, et al. Porous $\mathrm{NiCo}_{2} \mathrm{~S}_{4}$ nanoneedle arrays with highly efficient electrocatalysis anchored on carbon cloths as selfsupported hosts for high-loading Li-S batteries. ACS Appl Mater Interfaces, 2020, 12: 57975-57986

164 Zhang S, Ai Y, Wu SC, et al. 3D CoMoSe ${ }_{4}$ nanosheet arrays converted directly from hydrothermally processed $\mathrm{CoMoO}_{4}$ nanosheet arrays by plasma-assisted selenization process toward excellent anode material in sodium-ion battery. Nanoscale Res Lett, 2019, 14: 213

165 Ai Z, Hu Z, Liu Y, et al. Capacitance performance of nanostructured $\mathrm{CoNi}_{2} \mathrm{~S}_{4}$ with different morphology grown on carbon cloth for supercapacitors. ChemPlusChem, 2016, 81: 322-328

166 Abuali M, Arsalani N, Ahadzadeh I. Investigation of electrochemical performance of a new nanocomposite: $\mathrm{CuCo}_{2} \mathrm{~S}_{4} /$ polyaniline on carbon cloth. J Energy Storage, 2020, 32: 101694

$167 \mathrm{Hu} \mathrm{A}$, Long J, Shu C, et al. Three-dimensional $\mathrm{CoNi}_{2} \mathrm{~S}_{4}$ nanorod arrays anchored on carbon textiles as an integrated cathode for high-rate and long-life lithium-oxygen battery. Electrochim Acta, 2019, 301: 69-79

168 Sun M, Tie J, Cheng G, et al. In situ growth of burl-like nickel cobalt sulfide on carbon fibers as high-performance supercapacitors. J Mater Chem A, 2015, 3: 1730-1736

169 Cai X, Hansen RV, Zhang L, et al. Binary metal sulfides and polypyrrole on vertically aligned carbon nanotube arrays/carbon fiber paper as high-performance electrodes. J Mater Chem A, 2015, 3: 22043-22052

170 Xiong $\mathrm{X}$, Waller G, Ding D, et al. Controlled synthesis of $\mathrm{NiCo}_{2} \mathrm{~S}_{4}$ nanostructured arrays on carbon fiber paper for high-performance pseudocapacitors. Nano Energy, 2015, 16: 71-80

171 Yang W, Chen L, Yang J, et al. One-step growth of $3 \mathrm{D} \mathrm{CoNi}_{2} \mathrm{~S}_{4}$ nanorods and cross-linked $\mathrm{NiCo}_{2} \mathrm{~S}_{4}$ nanosheet arrays on carbon paper as anodes for high-performance lithium ion batteries. Chem Commun, 2016, 52: 5258-5261

172 Javed MS, Lei H, Li J, et al. Construction of highly dispersed mesoporous bimetallic-sulfide nanoparticles locked in $\mathrm{N}$-doped graphitic carbon nanosheets for high energy density hybrid flexible pseudocapacitors. J Mater Chem A, 2019, 7: 17435-17445

173 Manikandan R, Raj CJ, Nagaraju G, et al. Selective design of binderfree hierarchical nickel molybdenum sulfide as a novel battery-type material for hybrid supercapacitors. J Mater Chem A, 2019, 7: 2546725480

174 Ye B, Cao X, Zhao Q, et al. Electrodeposited $\mathrm{NiFe}_{2} \mathrm{Se}_{4}$ on nickel foam as a binder-free electrode for high-performance asymmetric supercapacitors. Ind Eng Chem Res, 2020, 59: 14163-14171

175 Shi X, Wang $\mathrm{H}$, Ji S, et al. CoNiSe ${ }_{2}$ nanorods directly grown on $\mathrm{Ni}$ foam as advanced cathodes for asymmetric supercapacitors. Chem Eng J, 2019, 364: 320-327

176 Sun J, Yao J, Liu Y, et al. Formation of hollow Co-Ni-S nanowedges arrays via sulfidation-etch of ZIF-L for advanced hybrid super- 
capacitor. ChemistrySelect, 2020, 5: 660-667

177 Cui Y, Zhang J, Jin C, et al. Ionic liquid-controlled growth of $\mathrm{NiCo}_{2} \mathrm{~S}_{4}$ 3D hierarchical hollow nanoarrow arrays on $\mathrm{Ni}$ foam for superior performance binder free hybrid supercapacitors. Small, 2018, 15: 1804318

178 Liu S, Kim KH, Yun JM, et al. 3D yolk-shell $\mathrm{NiGa}_{2} \mathrm{~S}_{4}$ microspheres confined with nanosheets for high performance supercapacitors. J Mater Chem A, 2017, 5: 6292-6298

179 Wang Y, Zhang W, Guo X, et al. Ni-Co selenide nanosheet/3D graphene/nickel foam binder-free electrode for high-performance supercapacitor. ACS Appl Mater Interfaces, 2019, 11: 7946-7953

180 Ye B, Cao X, Zhao Q, et al. Free-standing $\mathrm{NiCoSe}_{2}$ nanostructure on $\mathrm{Ni}$ foam via electrodeposition as high-performance asymmetric supercapacitor electrode. Nanotechnology, 2020, 31: 335706

181 Hou L, Sun X, Guo L, et al. An aqueous battery-pseudocapacitor hybrid capacitor based on conductive core-shell $\mathrm{NiCoSe}_{2} @ \mathrm{Co}_{9} \mathrm{Se}_{8}$ hollow nanospheres hybridized with nanoscale $\mathrm{Ru}_{0.41} \mathrm{In}_{0.59} \mathrm{O}_{y}$. Energy Technol, 2019, 8: 1901319

182 Zhang H, Hao A, Sun Z, et al. Boosting the performance of half/full lithium-ion batteries by designing smart architecture anode of $\mathrm{SnS}_{2}$ nanosheet coating on $\mathrm{NiCo}_{2} \mathrm{~S}_{4}$ hollow spheres. J Alloys Compd, 2020, 847: 156505

183 Sonia YK, Paliwal MK, Meher SK. The rational design of hierarchical $\mathrm{CoS}_{2} / \mathrm{CuCo}_{2} \mathrm{~S}_{4}$ for three-dimensional all-solid-state hybrid supercapacitors with high energy density, rate efficiency, and operational stability. Sustain Energy Fuels, 2021, 5: 973-985

184 Yao M, Hu Z, Liu Y, et al. Design and synthesis of hierarchical $\mathrm{NiCo}_{2} \mathrm{~S}_{4} @ \mathrm{NiMoO}_{4}$ core/shell nanospheres for high-performance supercapacitors. New J Chem, 2015, 39: 8430-8438

185 Nguyen DC, Tran DT, Doan TLL, et al. Rational design of core@shell structured $\mathrm{CoS}_{x} @ \mathrm{Cu}_{2} \mathrm{MoS}_{4}$ hybridized $\mathrm{MoS}_{2} / \mathrm{N}, \mathrm{S}$-codoped graphene as advanced electrocatalyst for water splitting and Zn-air battery. Adv Energy Mater, 2020, 10: 1903289

186 Meng A, Yuan X, Shen T, et al. One-step synthesis of flower-like $\mathrm{Bi}_{2} \mathrm{O}_{3} / \mathrm{Bi}_{2} \mathrm{Se}_{3}$ nanoarchitectures and $\mathrm{NiCoSe}_{2} / \mathrm{Ni}_{0.85} \mathrm{Se}$ nanoparticles with appealing rate capability for the construction of high-energy and long-cycle-life asymmetric aqueous batteries. J Mater Chem A, 2019, 7: 17613-17625

187 Ma F, Dai X, Jin J, et al. Hierarchical core-shell hollow CoMoS $@$ @NiCo-S nanotubes hybrid arrays as advanced electrode material for supercapacitors. Electrochim Acta, 2020, 331: 135459

188 Zhou S, Liu Y, Yan M, et al. Design of $\mathrm{FeCo}_{2} \mathrm{~S}_{4} @ \mathrm{Ni}(\mathrm{OH})_{2}$ core-shell hollow nanotube arrays on carbon paper for ultra-high capacitance in supercapacitors. Electrochim Acta, 2020, 349: 136337

189 Yan Y, Li A, Lu C, et al. Double-layered yolk-shell microspheres with $\mathrm{NiCo}_{2} \mathrm{~S}_{4}-\mathrm{Ni}_{9} \mathrm{~S}_{8}-\mathrm{C}$ hetero-interfaces as advanced battery-type electrode for hybrid supercapacitors. Chem Eng J, 2020, 396: 125316

190 Zhao X, Song Y, Liu ZH. Kinetics enhanced hierarchical $\mathrm{Ni}_{2} \mathrm{P}_{1-x} \mathrm{~S}_{x} /$ Ni@carbon/graphene yolk-shell microspheres boosting advanced sodium/potassium storage. J Mater Chem A, 2020, 8: 23994-24004

191 Wan L, He C, Chen D, et al. In situ grown $\mathrm{NiFeP@NiCo} \mathrm{S}_{4}$ nanosheet arrays on carbon cloth for asymmetric supercapacitors. Chem Eng J, 2020, 399: 125778

$192 \mathrm{Li} J$, Zhao J, Qin L, et al. Hierarchical $\mathrm{Co}(\mathrm{OH})_{2} @ \mathrm{NiMoS}_{4}$ nanocomposite on carbon cloth as electrode for high-performance asymmetric supercapacitors. RSC Adv, 2020, 10: 22606-22615

193 Singh A, Ojha SK, Singh M, et al. Controlled synthesis of $\mathrm{NiCo}_{2} \mathrm{~S}_{4} @$ $\mathrm{NiCo}_{2} \mathrm{O}_{4}$ core@shell nanostructured arrays decorated over the rGO sheets for high-performance asymmetric supercapacitor. Electrochim Acta, 2020, 349: 136349

194 Huang Y, Cui F, Bao J, et al. $\mathrm{MnCo}_{2} \mathrm{~S}_{4} / \mathrm{FeCo}_{2} \mathrm{~S}_{4}$ "lollipop" arrays on a hollow $\mathrm{N}$-doped carbon skeleton as flexible electrodes for hybrid supercapacitors. J Mater Chem A, 2019, 7: 20778-20789

195 Cui M, Bai X, Zhu J, et al. Electrochemically induced $\mathrm{NiCoSe}_{2} @$ $\mathrm{NiOOH} / \mathrm{CoOOH}$ heterostructures as multifunctional cathode materials for flexible hybrid $\mathrm{Zn}$ batteries. Energy Storage Mater, 2021, 36: 427-434

196 Moradlou O, Sharifpour $\mathrm{H}$. Interconnected $\mathrm{NiCo}_{2} \mathrm{~S}_{4}$-coated $\mathrm{NiO}$ nanosheet arrays as electrode materials for high-performance super- capacitors. J Energy Storage, 2020, 32: 101886

197 Cao XJ, Zeng HY, Cao X, et al. 3D-hierarchical mesoporous $\mathrm{CuCo}_{2} \mathrm{~S}_{4} @ \mathrm{NiCoAl}$ hydrotalcite/Ni foam material for high-performance supercapacitors. Appl Clay Sci, 2020, 199: 105864

198 Lei X, Ge S, Yang TY, et al. Ni-Mo-S@Ni-P composite materials as binder-free electrodes for aqueous asymmetric supercapacitors with enhanced performance. J Power Sources, 2020, 477: 229022

199 Mohammadi Zardkhoshoui A, Hayati Monjoghtapeh R, Hosseiny Davarani SS. Zn-Ni-Se@ $\mathrm{NiCo}_{2} \mathrm{~S}_{4}$ core-shell architectures: A highly efficient positive electrode for hybrid supercapacitors. Energy Fuels, 2020, 34: 14934-14947

200 Wang M, Zhang L, He Y, et al. Recent advances in transition-metalsulfide-based bifunctional electrocatalysts for overall water splitting. J Mater Chem A, 2021, 9: 5320-5363

201 Tang $\mathrm{N}$, You $\mathrm{H}$, Li $\mathrm{M}$, et al. Cross-linked $\mathrm{Ni}(\mathrm{OH})_{2} / \mathrm{CuCo}_{2} \mathrm{~S}_{4} / \mathrm{Ni}$ networks as binder-free electrodes for high performance supercapatteries. Nanoscale, 2018, 10: 20526-20532

202 Li S, Huang W, Yang Y, et al. Hierarchical layer-by-layer porous $\mathrm{FeCo}_{2} \mathrm{~S}_{4} @ \mathrm{Ni}(\mathrm{OH})_{2}$ arrays for all-solid-state asymmetric supercapacitors. J Mater Chem A, 2018, 6: 20480-20490

203 Huang Y, Ge S, Chen X, et al. Hierarchical $\mathrm{FeCo}_{2} \mathrm{~S}_{4} @ \mathrm{FeNi}_{2} \mathrm{~S}_{4}$ core/ shell nanostructures on $\mathrm{Ni}$ foam for high-performance supercapacitors. Chem Eur J, 2019, 25: 14117-14122

204 Khaja Hussain S, Vamsi Krishna BN, Nagaraju G, et al. Porous Co$\mathrm{MoS}_{2} @ \mathrm{Cu}_{2} \mathrm{MoS}_{4}$ three-dimensional nanoflowers via in situ sulfurization of $\mathrm{Cu}_{2} \mathrm{O}$ nanospheres for electrochemical hybrid capacitors. Chem Eng J, 2021, 403: 126319

205 Lim D, Lim C, Hwang M, et al. Facile synthesis of flower-like P-doped nickel-iron disulfide microspheres as advanced electrocatalysts for the oxygen evolution reaction. J Power Sources, 2021, 490: 229552

206 Lin J, Wang Y, Zheng $\mathrm{X}$, et al. P-doped $\mathrm{NiCo}_{2} \mathrm{~S}_{4}$ nanotubes as batterytype electrodes for high-performance asymmetric supercapacitors. Dalton Trans, 2018, 47: 8771-8778

$207 \mathrm{Gu} \mathrm{H}$, Fan W, Liu T. Phosphorus-doped $\mathrm{NiCo}_{2} \mathrm{~S}_{4}$ nanocrystals grown on electrospun carbon nanofibers as ultra-efficient electrocatalysts for the hydrogen evolution reaction. Nanoscale Horiz, 2017, 2: 277-283

208 Min K, Yoo R, Kim S, et al. Facile synthesis of P-doped $\mathrm{NiCo}_{2} \mathrm{~S}_{4}$ nanoneedles supported on $\mathrm{Ni}$ foam as highly efficient electrocatalysts for alkaline oxygen evolution reaction. Electrochim Acta, 2021, 396: 139236

209 Zong Q, Zhu Y, Wang Q, et al. Prussian blue analogues anchored P$(\mathrm{Ni}, \mathrm{Co}) \mathrm{Se}_{2}$ nanoarrays for high performance all-solid-state supercapacitor. Chem Eng J, 2020, 392: 123664

210 Lin J, Zhong Z, Wang $\mathrm{H}$, et al. Rational constructing free-standing Se doped nickel-cobalt sulfides nanotubes as battery-type electrode for high-performance supercapattery. J Power Sources, 2018, 407: 6-13

211 Kim J, Tabassian R, Nguyen VH, et al. Crumpled quaternary nanoarchitecture of sulfur-doped nickel cobalt selenide directly grown on carbon cloth for making stronger ionic soft actuators. ACS Appl Mater Interfaces, 2019, 11: 40451-40460

212 Phonsuksawang $\mathrm{P}$, Khajondetchairit P, Butburee T, et al. Effects of Fe doping on enhancing electrochemical properties of $\mathrm{NiCo}_{2} \mathrm{~S}_{4}$ supercapacitor electrode. Electrochim Acta, 2020, 340: 135939

213 Phonsuksawang P, Khajondetchairit P, Ngamchuea K, et al. Enhancing performance of $\mathrm{NiCo}_{2} \mathrm{~S}_{4} / \mathrm{Ni}_{3} \mathrm{~S}_{2}$ supercapacitor electrode by $\mathrm{Mn}$ doping. Electrochim Acta, 2021, 368: 137634

214 Peng Z, Yang C, Hu Y, et al. Double-shelled Mn-doped $\mathrm{NiCo}_{2} \mathrm{~S}_{4}$ hollow nanowire arrays for high-reactivity hybrid supercapacitors. Appl Surf Sci, 2022, 573: 151561

215 Wang Y, Chen N, Du X, et al. Transition metal atoms $\mathrm{M}(\mathrm{M}=\mathrm{Mn}, \mathrm{Fe}$, $\mathrm{Cu}, \mathrm{Zn}$ ) doped nickel-cobalt sulfides on the $\mathrm{Ni}$ foam for efficient oxygen evolution reaction and urea oxidation reaction. J Alloys Compd, 2022, 893: 162269

216 Nagaraju G, Sekhar SC, Ramulu B, et al. High-performance hybrid supercapacitors based on MOF-derived hollow ternary chalcogenides. Energy Storage Mater, 2021, 35: 750-760

217 Lai F, Feng J, Heil T, et al. Partially delocalized charge in Fe-doped $\mathrm{NiCo}_{2} \mathrm{~S}_{4}$ nanosheet-mesoporous carbon-composites for high-voltage supercapacitors. J Mater Chem A, 2019, 7: 19342-19347 
218 Zhang L, Zhang H, Jin L, et al. Composition controlled nickel cobalt sulfide core-shell structures as high capacity and good rate-capability electrodes for hybrid supercapacitors. RSC Adv, 2016, 6: 50209-50216

219 Ai Z, Hu Z, Liu Y, et al. Novel 3D flower-like $\mathrm{CoNi}_{2} \mathrm{~S}_{4} /$ carbon nanotube composites as high-performance electrode materials for supercapacitors. New J Chem, 2016, 40: 340-347

220 Yang $\mathrm{X}, \mathrm{He} \mathrm{X}, \mathrm{Li} \mathrm{Q}$, et al. 3D hierarchical $\mathrm{NiCo}_{2} \mathrm{~S}_{4}$ nanoparticles/ carbon nanotube sponge cathode for highly compressible asymmetric supercapacitors. Energy Fuels, 2021, 35: 3449-3458

$221 \mathrm{Hu} \mathrm{Q}, \mathrm{Ma} \mathrm{W}$, Liang G, et al. Anion-exchange reaction synthesized $\mathrm{CoNi}_{2} \mathrm{~S}_{4}$ nanowires for superior electrochemical performances. RSC Adv, 2015, 5: 84974-84979

222 Chen $\mathrm{H}$, Jiang J, Zhang $\mathrm{L}$, et al. In situ growth of $\mathrm{NiCo}_{2} \mathrm{~S}_{4}$ nanotube arrays on $\mathrm{Ni}$ foam for supercapacitors: Maximizing utilization efficiency at high mass loading to achieve ultrahigh areal pseudocapacitance. J Power Sources, 2014, 254: 249-257

$223 \mathrm{Pu}$ J, Wang $\mathrm{T}$, Wang $\mathrm{H}$, et al. Direct growth of $\mathrm{NiCo}_{2} \mathrm{~S}_{4}$ nanotube arrays on nickel foam as high-performance binder-free electrodes for supercapacitors. ChemPlusChem, 2014, 79: 577-583

224 Moosavifard SE, Fani S, Rahmanian M. Hierarchical $\mathrm{CuCo}_{2} \mathrm{~S}_{4}$ hollow nanoneedle arrays as novel binder-free electrodes for high-performance asymmetric supercapacitors. Chem Commun, 2016, 52: 45174520

225 Pramanik A, Maiti S, Sreemany M, et al. Carbon doped $\mathrm{MnCo}_{2} \mathrm{~S}_{4}$ microcubes grown on Ni foam as high energy density Faradaic electrode. Electrochim Acta, 2016, 213: 672-679

226 Kong W, Lu C, Zhang W, et al. Homogeneous core-shell $\mathrm{NiCo}_{2} \mathrm{~S}_{4}$ nanostructures supported on nickel foam for supercapacitors. J Mater Chem A, 2015, 3: 12452-12460

227 Yi TF, Pan JJ, Wei TT, et al. $\mathrm{NiCo}_{2} \mathrm{~S}_{4}$-based nanocomposites for energy storage in supercapacitors and batteries. Nano Today, 2020, 33: 100894

228 Gong J, Yang J, Wang J, et al. A dual NiCo metal-organic frameworks derived $\mathrm{NiCo}_{2} \mathrm{~S}_{4}$ core-shell nanorod arrays as high-performance electrodes for asymmetric supercapacitors. Electrochim Acta, 2021, 374: 137794

229 Nagaraju G, Sekhar SC, Ramulu B, et al. An integrated approach toward renewable energy storage using rechargeable $\mathrm{Ag@Ni} \mathrm{Ni}_{0.67} \mathrm{Co}_{0.33} \mathrm{~S}$ based hybrid supercapacitors. Small, 2019, 15: 1805418

230 Xiao J, Wan L, Yang S, et al. Design hierarchical electrodes with highly conductive $\mathrm{NiCo}_{2} \mathrm{~S}_{4}$ nanotube arrays grown on carbon fiber paper for high-performance pseudocapacitors. Nano Lett, 2014, 14: 831-838

231 Niu L, Wang Y, Ruan F, et al. In situ growth of $\mathrm{NiCo}_{2} \mathrm{~S}_{4} @ \mathrm{Ni}_{3} \mathrm{~V}_{2} \mathrm{O}_{8}$ on $\mathrm{Ni}$ foam as a binder-free electrode for asymmetric supercapacitors. J Mater Chem A, 2016, 4: 5669-5677

232 Hussain I, Ali A, Lamiel C, et al. A 3D walking palm-like core-shell $\mathrm{CoMoO}_{4} @ \mathrm{NiCo}_{2} \mathrm{~S}_{4} @$ nickel foam composite for high-performance supercapacitors. Dalton Trans, 2019, 48: 3853-3861

233 Miao F, Li X, Tao B, et al. Heterostructured $\mathrm{Co}(\mathrm{OH})_{2}$ nanosheetcoated $\mathrm{CuCo}_{2} \mathrm{~S}_{4}$ nanopencils on nickel foam for electrodes in highperformance supercapacitors. Ionics, 2020, 26: 5241-5249

234 He Q, Yang T, Wang X, et al. Metal-organic framework derived hierarchical zinc nickel selenide/nickel hydroxide microflower supported on nickel foam with enhanced electrochemical properties for supercapacitor. J Mater Sci-Mater Electron, 2021, 32: 3649-3660

235 Wan $\mathrm{H}$, Liu J, Ruan $\mathrm{Y}$, et al. Hierarchical configuration of $\mathrm{NiCo}_{2} \mathrm{~S}_{4}$ nanotube@Ni-Mn layered double hydroxide arrays/three-dimensional graphene sponge as electrode materials for high-capacitance supercapacitors. ACS Appl Mater Interfaces, 2015, 7: 15840-15847

236 Ameri B, Zardkhoshoui AM, Hosseiny Davarani SS. Engineering of hierarchical $\mathrm{NiCoSe}_{2} @ \mathrm{NiMn}-\mathrm{LDH}$ core-shell nanostructures as a high-performance positive electrode material for hybrid supercapacitors. Sustain Energy Fuels, 2020, 4: 5144-5155

237 Tao Y, Ruiyi L, Lin Z, et al. Three-dimensional electrode of $\mathrm{Ni} / \mathrm{Co}$ layered double hydroxides@ $\mathrm{NiCo}_{2} \mathrm{~S}_{4} @ g r a p h e n e @ \mathrm{Ni}$ foam for supercapacitors with outstanding electrochemical performance. Electrochim Acta, 2015, 176: 1153-1164

238 Yang B, Yu L, Yan $\mathrm{H}$, et al. Fabrication of urchin-like $\mathrm{NiCo}_{2}\left(\mathrm{CO}_{3}\right)_{1.5-}$ $(\mathrm{OH})_{3} @ \mathrm{NiCo}_{2} \mathrm{~S}_{4}$ on Ni foam by an ion-exchange route and application to asymmetrical supercapacitors. J Mater Chem A, 2015, 3: 1330813316

239 Fu W, Zhao C, Han W, et al. Cobalt sulfide nanosheets coated on $\mathrm{NiCO}_{2} \mathrm{~S}_{4}$ nanotube arrays as electrode materials for high-performance supercapacitors. J Mater Chem A, 2015, 3: 10492-10497

240 Yang X, Sun $\mathrm{H}$, Zan P, et al. Growth of vertically aligned $\mathrm{Co}_{3} \mathrm{~S}_{4} /$ $\mathrm{CoMo}_{2} \mathrm{~S}_{4}$ ultrathin nanosheets on reduced graphene oxide as a highperformance supercapacitor electrode. J Mater Chem A, 2016, 4 18857-18867

241 Hou L, Shi Y, Zhu S, et al. Hollow mesoporous hetero- $\mathrm{NiCo}_{2} \mathrm{~S}_{4} / \mathrm{Co}_{9} \mathrm{~S}_{8}$ submicro-spindles: Unusual formation and excellent pseudocapacitance towards hybrid supercapacitors. J Mater Chem A, 2017, 5: 133144

242 Shen J, Dong P, Baines R, et al. Controlled synthesis and comparison of $\mathrm{NiCo}_{2} \mathrm{~S}_{4} /$ graphene/2D TMD ternary nanocomposites for highperformance supercapacitors. Chem Commun, 2016, 52: 9251-9254

243 Shen J, Wu J, Pei L, et al. $\mathrm{CoNi}_{2} \mathrm{~S}_{4}$-graphene-2D-MoSe 2 as an advanced electrode material for supercapacitors. Adv Energy Mater, 2016, 6: 1600341

244 Li S. Bimetallic cobalt-nickel selenide@polypyrrole core-shell nanotubes on nickel foam as high-performance electrode material for supercapacitors. Int J Electrochem Sci, 2020, 15: 2923-2934

245 Yan $\mathrm{M}$, Yao $\mathrm{Y}$, Wen $\mathrm{J}$, et al. Construction of a hierarchical $\mathrm{NiCo}_{2} \mathrm{~S}_{4} @ \mathrm{PPy}$ core-shell heterostructure nanotube array on $\mathrm{Ni}$ foam for a high-performance asymmetric supercapacitor. ACS Appl Mater Interfaces, 2016, 8: 24525-24535

246 Zhu J, Tang $\mathrm{S}$, Wu J, et al. Wearable high-performance supercapacitors based on silver-sputtered textiles with $\mathrm{FeCo}_{2} \mathrm{~S}_{4}-\mathrm{NiCo}_{2} \mathrm{~S}_{4}$ composite nanotube-built multitripod architectures as advanced flexible electrodes. Adv Energy Mater, 2017, 7: 1601234

247 Huang X, Gou L. Design and synthesis of 3D hierarchical NiMoS $@$ $\mathrm{CuCo}_{2} \mathrm{~S}_{4}$ array electrode with excellent electrochemical performance. Nanotechnology, 2020, 31: 185602

248 He Y, Luo M, Dong C, et al. Coral-like $\mathrm{Ni}_{x} \mathrm{Co}_{1-x} \mathrm{Se}_{2}$ for Na-ion battery with ultralong cycle life and ultrahigh rate capability. J Mater Chem A, 2019, 7: 3933-3940

249 Jin $\mathrm{R}$, Liu D, Liu C, et al. Hierarchical $\mathrm{NiCo}_{2} \mathrm{~S}_{4}$ hollow spheres as a high performance anode for lithium ion batteries. RSC Adv, 2015, 5 84711-84717

250 Zhao Y, Manthiram A. $\mathrm{Bi}_{0.94} \mathrm{Sb}_{1.06} \mathrm{~S}_{3}$ nanorod cluster anodes for sodium-ion batteries: Enhanced reversibility by the synergistic effect of the $\mathrm{Bi}_{2} \mathrm{~S}_{3}-\mathrm{Sb}_{2} \mathrm{~S}_{3}$ solid solution. Chem Mater, 2015, 27: 6139-6145

251 Zou R, Zhang Z, Yuen MF, et al. Three-dimensional-networked $\mathrm{NiCo}_{2} \mathrm{~S}_{4}$ nanosheet array/carbon cloth anodes for high-performance lithium-ion batteries. NPG Asia Mater, 2015, 7: e195

252 Fu L, Wang X, Ma J, et al. Graphene-encapsulated copper tin sulfide submicron spheres as high-capacity binder-free anode for lithium-ion batteries. ChemElectroChem, 2017, 4: 1124-1129

253 Zhang $\mathrm{L}$, Zuo L, Fan W, et al. $\mathrm{NiCo}_{2} \mathrm{~S}_{4}$ nanosheets grown on $3 \mathrm{D}$ networks of nitrogen-doped graphene/carbon nanotubes: Advanced anode materials for lithium-ion batteries. ChemElectroChem, 2016, 3: 1384-1391

254 Li Q, Jiao Q, Zhou W, et al. Controllable construction of core-shell $\mathrm{CuCo}_{2} \mathrm{~S}_{4} @$ polypyrrole nanocomposites as advanced anode materials for high-performance sodium ion half/full batteries. Mater Chem Front, 2021, 5: 293-303

255 Bai D, Wang F, Lv J, et al. Triple-confined well-dispersed biactive $\mathrm{NiCo}_{2} \mathrm{~S}_{4} / \mathrm{Ni}_{0.96} \mathrm{~S}$ on graphene aerogel for high-efficiency lithium storage. ACS Appl Mater Interfaces, 2016, 8: 32853-32861

256 Yu DJ, Yuan YF, Zhang D, et al. Nickel cobalt sulfide nanotube array on nickel foam as anode material for advanced lithium-ion batteries. Electrochim Acta, 2016, 198: 280-286

257 Ren QQ, Liu C, Wang ZB, et al. 3D $\mathrm{NiCo}_{2} \mathrm{~S}_{4}$ nanorod arrays as electrode materials for electrochemical energy storage application. Ceramics Int, 2016, 42: 18173-18180

258 Wang JG, Jin $\mathrm{D}$, Zhou $\mathrm{R}$, et al. One-step synthesis of $\mathrm{NiCo}_{2} \mathrm{~S}_{4}$ ultrathin nanosheets on conductive substrates as advanced electrodes for highefficient energy storage. J Power Sources, 2016, 306: 100-106

259 Zhang J, Song K, Mi L, et al. Bimetal synergistic effect induced high 
reversibility of conversion-type $\mathrm{Ni@NiCo} \mathrm{S}_{4}$ as a free-standing anode for sodium ion batteries. J Phys Chem Lett, 2020, 11: 1435-1442

260 Liang Y, Dong H, Aurbach D, et al. Current status and future directions of multivalent metal-ion batteries. Nat Energy, 2020, 5: 646-656

261 Yousaf M, Naseer U, Li Y, et al. A mechanistic study of electrode materials for rechargeable batteries beyond lithium ions by in situ transmission electron microscopy. Energy Environ Sci, 2021, 14: 2670-2707

$262 \mathrm{Xu} \mathrm{X}$, Chao D, Chen B, et al. Revealing the magnesium-storage mechanism in mesoporous bismuth via spectroscopy and ab-initio simulations. Angew Chem Int Ed, 2020, 59: 21728-21735

263 Hao J, Yuan L, Johannessen B, et al. Studying the conversion mechanism to broaden cathode options in aqueous zinc-ion batteries. Angew Chem Int Ed, 2021, 60: 25114-25121

264 Wang M, Lai Y, Fang J, et al. Hydrangea-like $\mathrm{NiCo}_{2} \mathrm{~S}_{4}$ hollow microspheres as an advanced bifunctional electrocatalyst for aqueous metal/air batteries. Catal Sci Technol, 2016, 6: 434-437

265 Wang S, Suo Y, Su C, et al. Graphene decorated with multiple nanosized active species as dual function electrocatalysts for lithiumoxygen batteries. Electrochim Acta, 2016, 188: 718-726

266 Huang M, Li M, Niu C, et al. Recent advances in rational electrode designs for high-performance alkaline rechargeable batteries. Adv Funct Mater, 2019, 29: 1807847

267 Zhu Y, Li J, Yun X, et al. Graphitic carbon quantum dots modified nickel cobalt sulfide as cathode materials for alkaline aqueous batteries. Nano-Micro Lett, 2020, 12: 16

268 He G, Qiao M, Li W, et al. S, N-Co-doped graphene-nickel cobalt sulfide aerogel: Improved energy storage and electrocatalytic performance. Adv Sci, 2017, 4: 1600214

269 Fei B, Yao Z, Cai D, et al. Construction of sugar gourd-like yolk-shell Ni-Mo-Co-S nanocage arrays for high-performance alkaline battery. Energy Storage Mater, 2020, 25: 105-113

270 Li W, Zhang B, Lin R, et al. A dendritic nickel cobalt sulfide nanostructure for alkaline battery electrodes. Adv Funct Mater, 2018, 28: 1705937

271 Chen B, Chao D, Liu E, et al. Transition metal dichalcogenides for alkali metal ion batteries: Engineering strategies at the atomic level. Energy Environ Sci, 2020, 13: 1096-1131

Acknowledgements This work was supported by the National Natural Science Fund for Distinguished Young Scholars (52025133), the Tencent Foundation through the XPLORER PRIZE, Beijing Natural Science Foundation (JQ18005), the National Natural Science Foundation of China (52125307 and 11974023), the Fund of the State Key Laboratory of Solidification Processing in Northwestern Polytechnic University (NWPU) (SKLSP202004), and the Key Area R\&D Program of Guangdong Province (2018B030327001 and 2018B010109009).

Author contributions Yousaf M, Naseer U, and Guo S conceived the idea and led the whole project. Yousaf $\mathrm{M}$ and Naseer $\mathrm{U}$ wrote the manuscript. $\mathrm{Li}$ Y, Mahmood A, Ali I, Aftab W, and Mahmood N helped in collecting literature, writing and revising the manuscript. Gao P and Jiang Y helped in explaining the basics of MBCs for EES systems. Guo S provided mentorship to complete the project. All authors contributed to writing, discussing, and revising the manuscript.

Conflict of interest The authors declare that they have no conflict of interest.

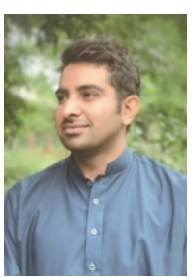

Muhammad Yousaf is a principal investigator (PI) at Hangzhou Global Scientific and Innovation Center, Zhejiang University, Hangzhou, China. He completed his PhD degree in advanced materials and mechanics from Peking University, China (2014-2019). He was a postdoctoral international exchange postdoctorate fellow at Peking University (2019-2021). His research includes the synthesis of transition metal chalcogenide-based heterostructures for rechargeable ion batteries with a particular interest in in-situ characterization of these materials using advanced TEM.

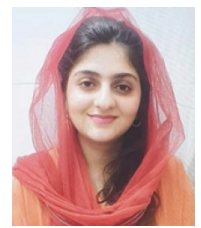

Ufra Naseer is pursuing her Master leading to $\mathrm{PhD}$ degree under the supervision of Professor Tianxiang Yue at the University of Chinese Academy of Sciences (UCAS), Beijing, China. She completed her undergraduate degree (BSc) in botany from Govt. College Women University Sialkot, Pakistan (2013-2017). Since 2020 she has been studying for her Master leading to $\mathrm{PhD}$ degree with an ANSO (The Alliance of International Science Organizations) scholarship. Her research includes environment and climate change.

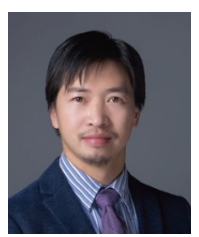

Peng Gao is currently a Professor at the School of Physics, Peking University, Beijing, China. He received his BSc degree in physics from the Uniersity of Science and Technology of China and $\mathrm{PhD}$ degree from the Institute of Physics, Chinese Academy of Sciences. Before he joined in Peking University in 2015, he was a post-doctoral fellow at the University of Michigan, a research associate at Brookhaven National Laboratory, and a research fellow at the University of Tokyo. His research interests are interface properties in ferroelectrics, solid-state ionics, and lightelement nanostructures.

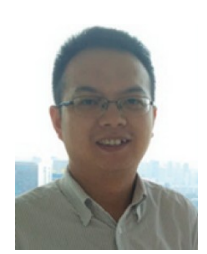

Yinzhu Jiang is a Professor at the School of Materials Science and Engineering, Zhejiang University, China. He received his $\mathrm{PhD}$ degree from the Department of Materials Science and Engineering, University of Science and Technology of China in 2007. He worked as a post-doctoral researcher at Heriot-Watt University, United Kingdom from 2007 to 2008 and an Alexander von Humboldt Fellow at Bielefeld University, Germany from 2008 to 2010. His research interests mainly focus on energy-related materials and electrochemistry, including rechargeable batteries, metal anodes and solid electrolytes.

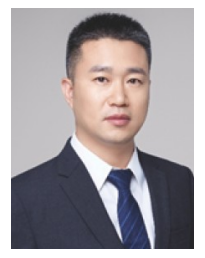

Shaojun Guo is a Boya Distinguished Professor at the School of Materials Science and Engineering, Peking University and a fellow of the Royal Society of Chemistry (RSC). Guo holds a BSc degree in materials chemistry from Jilin University and a $\mathrm{PhD}$ degree in analytical chemistry from Changchun Institute of Applied Chemistry, Chinese Academy of Sciences. Before he was appointed a faculty member of Peking University in 2015, he did his postdoctoral research at Brown University and was Oppenheimer fellow at Los Alamos National Lab. His current research interests are nano/sub-nano/atomic materials for catalysis and energy applications. 


\section{二元金属硫族化合物在能源储存体系中的作用: 挑战 和可能的解决策略}

Yousaf Muhammad ${ }^{1,2}$, Naseer Ufra ${ }^{3}$, Ali Imran ${ }^{2}$, 李一举 ${ }^{1}$, Aftab Waseem ${ }^{1}$, Mahmood Asif ${ }^{4}$, Mahmood Nasir ${ }^{5}$, 高鹏 ${ }^{1 *}$, 姜银珠 ${ }^{2,6^{*}}$, 郭少军 ${ }^{1 *}$

摘要 二元金属硫族化合物由于其丰富的相界面、高活性位点、优异 的导电性以及快速的电化学动力学, 与单金属硫族化合物相比具有更 加优异的电化学性能. 然而, 材料的本征离子电导率低, 在充放电过程 中结构发生反复的团聚与断裂, 体积膨胀大等因素导致其电化学性能 衰减严重. 为了解决上述问题, 诸多策略被提出并用于调控二元金属硫 族化合物的纳米结构以获得优化的电极材料. 但是目前依旧缺乏对二 元金属硫族化合物电化学过程的系统认识, 也因此限制了其大规模的 商业化应用. 在这篇综述中, 我们不仅重点介绍了基于二元金属硫族化 合物电极材料制备的最新研究进展, 还通过解释二元金属硫族化合物 的电化学动力学, 进一步构建和理解了这类材料的构效关系. 此外, 我 们讨论了通过纳米结构化和使用碳材料与三维模板与之形成复合材料 的策略来调控和修饰二元金属硫族化合物, 并将详细讨论二元金属硫 族化合物在超级电容器、金属离子电池、金属空气电池以及碱金属电 池等电化学能源储存体系中的工作机理. 最后, 我们总结了二元金属硫 族化合物在发展实际能源器件的过程中面临的主要挑战及可能的解决 策略. 我们相信, 这篇综述将对如何调控二元金属硫族化合物的理化性 质以获得优异的电化学器件提供指导性建议. 